\title{
On the mechanics of biopolymer networks
}

\author{
Dissertation \\ zur Erlangung des \\ mathematisch-naturwissenschaftlichen Doktorgrades \\ "Doctor rerum naturalium" \\ der Georg-August-Universität Göttingen \\ im Promotionsprogramm Physik \\ der Georg-August University School of Science (GAUSS) \\ vorgelegt von \\ Knut Heidemann \\ aus Hameln
}

Göttingen, 2016 


\section{Betreuungsausschuss:}

- Prof. Dr. Max Wardetzky,

Institut für Numerische und Angewandte Mathematik,

Georg-August-Universität Göttingen

- Prof. Dr. Christoph F. Schmidt,

Drittes Physikalisches Institut — Biophysik,

Georg-August-Universität Göttingen

\section{Prüfungskommission:}

- Referent:

Prof. Dr. Max Wardetzky,

Institut für Numerische und Angewandte Mathematik,

Georg-August-Universität Göttingen

- Korreferent:

Prof. Dr. Christoph F. Schmidt,

Drittes Physikalisches Institut - Biophysik,

Georg-August-Universität Göttingen

\section{Weitere Mitglieder der Prüfungskommission:}

- Prof. Dr. Jörg Enderlein, Drittes Physikalisches Institut — Biophysik, Georg-August-Universität Göttingen

- Prof. Dr. Helmut Grubmüller, Abteilung Theoretische und Computergestützte Biophysik, Max-Planck-Institut für Biophysikalische Chemie, Göttingen

- Prof. Dr. Stephan Huckemann, Institut für Mathematische Stochastik, Georg-August-Universität Göttingen

- Prof. Dr. Annette Zippelius, Institut für Theoretische Physik, Georg-August-Universität Göttingen

Tag der mündlichen Prüfung: 11.05.2016 


\section{Abstract}

In this thesis, we study the mechanical properties of biopolymer networks. We discuss which of these properties can be described by continuum approaches and which features, on the contrary, require consideration of the discrete nature or the topology of the network. For this purpose, we combine theoretical modeling with extensive numerical simulations.

In Chapter 2, we study the elasticity of disordered networks of rigid filaments connected by flexible crosslinks that are modeled as wormlike chains. Under the assumption of affine deformations in the limit of infinite crosslink density, we show analytically that the nonlinear elastic regime in 1- and 2-dimensional networks is characterized by power-law scaling of the elastic modulus with the stress. In contrast, 3-dimensional networks show an exponential dependence of the modulus on stress. Independent of dimensionality, if the crosslink density is finite, we show that the only persistent scaling exponent is that of the single wormlike chain. Our theoretical considerations are accompanied by extensive quasistatic simulations of 3-dimensional networks, which are in agreement with the analytical theory, and show additional features like prestress and the formation of force chains.

In Chapter 3, we study the distribution of forces in random spring networks on the unit circle by applying a combination of probabilistic theory and numerical computations. Using graph theory, we find that taking into account network topology is crucial to correctly capture force distributions in mechanical equilibrium. In particular, we show that application of a mean field approach results in significant deviations from the correct solution, especially for sparsely connected networks. 



\section{Citations to related work}

This thesis contains results from published and unpublished work of the author and collaborators. Chapter 2 is based on the published work:

- K. M. Heidemann, A. Sharma, F. Rehfeldt, C. F. Schmidt \& M. Wardetzky. Elasticity of 3D networks with rigid filaments and compliant crosslinks. Soft Matter 11, 343-354 (2015).

Additional text passages taken from the aforementioned publication are part of the abstract of this thesis.

Chapter 3 is based on the unpublished manuscript:

- K. M. Heidemann, A. O. Sageman-Furnas, A. Sharma, F. Rehfeldt, C. F. Schmidt \& M. Wardetzky. Random spring networks on the unit circle (in preparation).

Appendix A contains excerpts from the author's master's thesis:

- K. M. Heidemann. Network elasticity of stiff rods connected by flexible linkers Master's Thesis (Georg-August-University Göttingen, 2012). 



\section{Contents}

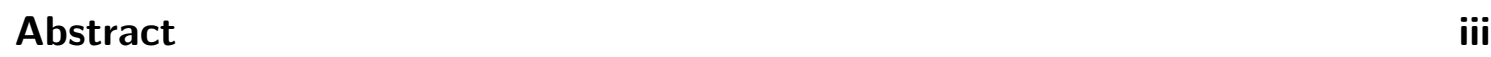

Citations to related work v

$\begin{array}{ll}\text { 1. Introduction } & 1\end{array}$

1.1. Biopolymers . . . . . . . . . . . . . . . . . . 1

1.2. Biopolymer networks . . . . . . . . . . . . . . . . . . 6

1.2.1. Experimental studies . . . . . . . . . . . . . . 8

1.2.2. Modeling strategies . . . . . . . . . . . . . . 11

1.3. Outline of the thesis . . . . . . . . . . . . . . . . . . 14

2. Networks with rigid filaments and compliant crosslinks 15

2.1. Key results . . . . . . . . . . . . . . . . . . 15

2.2. Publication . . . . . . . . . . . . . . . . . . 22

2.2.1. Introduction . . . . . . . . . . . . . . . 22

2.2 .2$. Theory . . . . . . . . . . . . . . . . 24

2.2.3. Simulation model . . . . . . . . . . . . . . . . . . . 30

2.2.4. Initial tension and prestress . . . . . . . . . . . . . . 34

2.2.5. Linear regime . . . . . . . . . . . . . . . . . . . 36

2.2.6. Nonlinear regime . . . . . . . . . . . . . . . 42

2.2.7. Conclusions . . . . . . . . . . . . . . . . . . . . 48

2.2.8. Acknowledgements . . . . . . . . . . . . 50

2.2.9. Appendix: Derivation of scaling relations for the shear modulus 50

3. Random spring networks on the unit circle 55

3.1. Key results . . . . . . . . . . . . . . . . . . 55

3.2. Manuscript . . . . . . . . . . . . . . . . . . . . 59

3.2.1. Introduction . . . . . . . . . . . . . . . . . . . . 59

3.2.2. Model and simulations . . . . . . . . . . . . . . . 60 
3.2.3. Probabilistic theory . . . . . . . . . . . . . . . 62

3.2.4. Conclusions . . . . . . . . . . . . . . . . 68

\begin{tabular}{ll}
\hline Summary \& Discussion & 71
\end{tabular}

\begin{tabular}{ll}
\hline A. Appendix & $\mathbf{7 5}$
\end{tabular}

A.1. The freely-jointed chain . . . . . . . . . . . . . . 75

A.2. The wormlike chain . . . . . . . . . . . . . . . . . . . . . . 78

A.3. Parseval's theorem . . . . . . . . . . . . . . . 83

A.4. Equipartition theorem . . . . . . . . . . . . . . 83

\begin{tabular}{ll}
\hline List of publications & 85
\end{tabular}

\begin{tabular}{lr}
\hline Bibliography & 90
\end{tabular}

\begin{tabular}{ll}
\hline Acknowledgments & 91
\end{tabular} 


\section{Introduction}

Polymeric materials are ubiquitous in our daily life: From rubber bands that we appreciate for their temporarily well behaved elasticity, to ourselves, who carry around a variety of slender molecules building up network structures like the cytoskeleton, which provides cell integrity, and the extracellular matrix, which fills the space in between the cells [1]. Biopolymer networks are usually highly dynamic and facilitate functions like cell division, cell motility or the transport of nutrients [1]. Furthermore, biopolymer networks possess exceptional mechanical properties, partly due to their composite nature, but also simply because they form disordered three-dimensional (3D) materials out of elongated one-dimensional (1D) molecules. It is our aim to gain a detailed understanding of the fundamental principles behind their material properties.

In this introduction, we first present what is known about the mechanical properties of individual biopolymers and then turn to network assemblies. We survey experimental studies on the elasticity of biopolymer networks and discuss some of the prominent modeling approaches in the field.

\subsection{Biopolymers}

Generally speaking, a polymer is a stringlike chain composed of similar subunits called monomers (see Fig. 1.1); usually, these subunits are of molecular size. Synthetic polymers can be found in daily materials like plastic (e.g., polystyrene or polyethylene), glue, etc. [2]. A well-known example for a biopolymer, i.e., a polymer produced by a living organism, is $D N A$, the carrier of our genetic code. It is composed of two polymer strands, with covalently bound monomeric units called nucleotides. Together they form the helically shaped double-stranded DNA. The fact that the genetic code is stored on these large macromolecules makes it robust (see [3] for an in-depth discussion). 


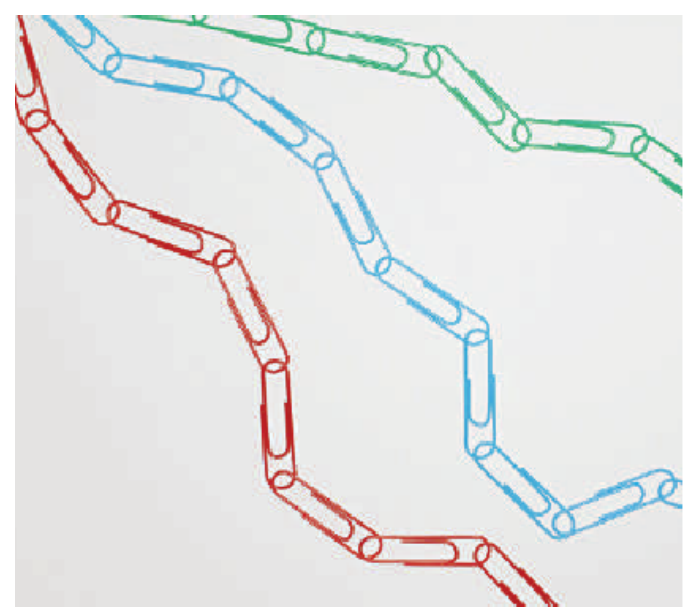

Figure 1.1.: Paperclip model showing how "polymers" are composed of individual "monomers" - here, paper clips. Figure taken from [4]. Reprinted by permission from Macmillan Publishers Ltd: Nature, copyright (2007)

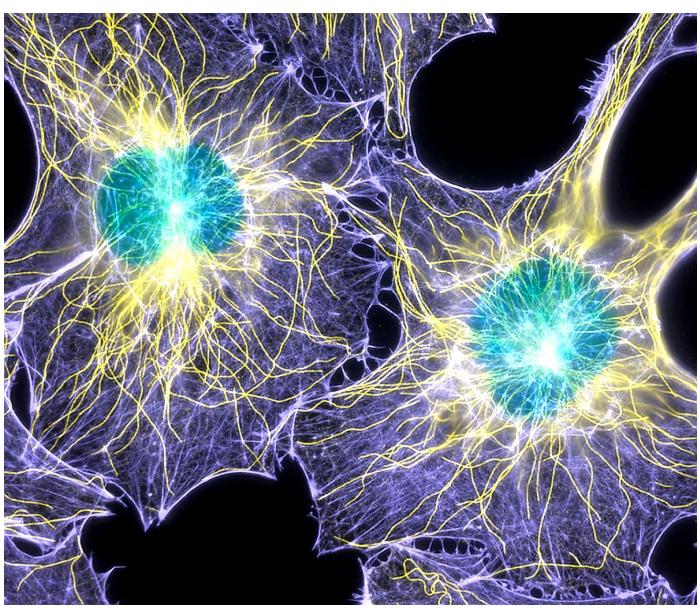

Figure 1.2.: Fibroblast cells with nuclei colored in green, microtubules in yellow, and actin filaments in purple. Figure taken from [5].

Another example are proteins; these are polymers consisting of a set of molecules called amino acids. The sequence of amino acids (the so-called primary structure) largely determines the properties of the protein and is encoded in the DNA. Many fold into characteristic shapes (secondary and tertiary structure), which are essential for their specific functioning, e.g., as an enzyme.

The major components that are involved when it comes to cell mechanics are polymers called cytoskeletal filaments. The cell's so-called cytoskeleton consists of essentially three different types: actin filaments, microtubules, and intermediate filaments, forming a highly entangled and crosslinked network (see Fig. 1.2). Each of them consists of proteins as monomeric subunits and in vivo they are highly dynamic structures, since their constituting elements are not covalently bound (in contrast to DNA). This possibility of polymerization and depolymerization (addition and removal of monomers) enables the cytoskeletal filaments to fascilitate dynamic processes like cell division and cell movement [1].

Within the context of this thesis we focus on the mechanical properties of polymeric materials. It has been shown that continuum elasticity can be applied to capture the mechanics of single polymer chains [6, 7], due to the fact that their length is usually much greater than the diameter of a single monomer. We therefore describe the polymer chain as a thin elastic rod, which, most generally, posesses three different 


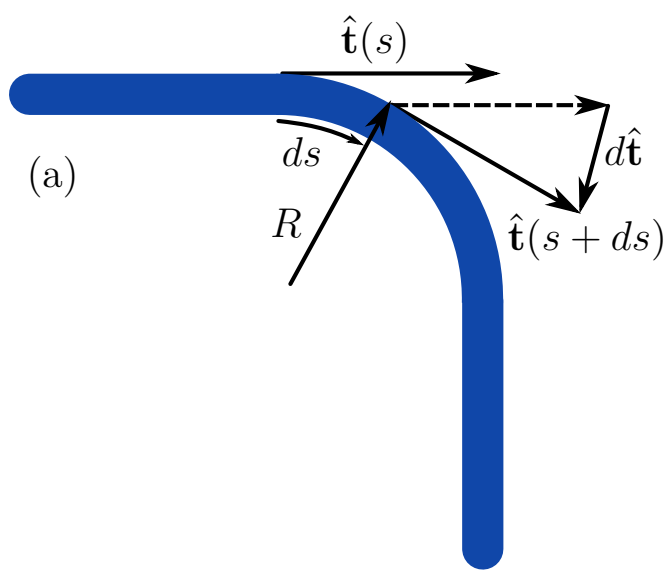

(b)

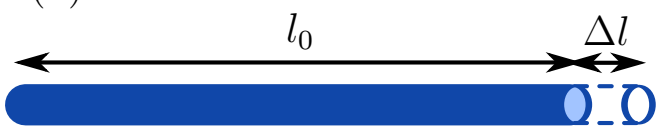

(c)

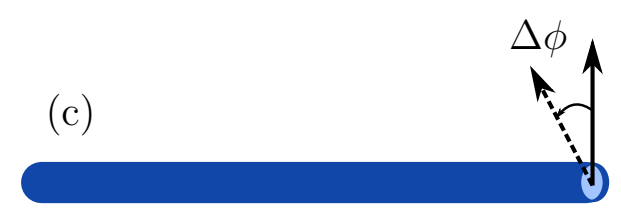

Figure 1.3.: Deformation modes of a thin elastic rod: (a) Bending: Curvature is measured via the rate of change of the unit tangent vector along the curve $d \hat{\mathbf{t}} / d s$; it equals in magnitude the inverse of the radius of curvature $1 / R$. (b) Stretching: A rod with intrinsic contour length $l_{0}$ is uniformly stretched by a total amount of $\Delta l$. (c) Twisting: While keeping the rod straight, it is uniformaly twisted about its axis by a total angle of $\Delta \phi$. Reproduced from 8 .

intrinsic deformation modes, i.e., bending, stretching/compression and twisting, which contribute to its elastic energy (see Fig. 1.3). Microscopically, these deformation modes correspond to changes in molecular binding angles or distances. Often, one can neglect the twist contribution, simply because the monomer bonds can rotate freely or the boundary conditions allow for twist relaxation [8]. Arguing that the energy contributions of an infinitesimal rod segment are quadratic [8], we can write for the total elastic energy of a naturally straight rod with contour length $l_{0}$ :

$$
\mathcal{E}_{\text {rod }}(\{\mathbf{r}(s)\})=\underbrace{\frac{A}{2} \int_{0}^{l_{0}}|d \hat{\mathbf{t}} / d s|^{2} d s}_{\text {bending }}+\underbrace{\frac{B}{2} \int_{0}^{l_{0}}\left(\left|\mathbf{r}^{\prime}(s)\right|-1\right)^{2} d s}_{\text {stretching }},
$$

where $\mathbf{r}(s)$ is the coordinate vector of the space curve describing the rod and $\hat{\mathbf{t}}(s)=\mathbf{r}^{\prime}(s) /\left|\mathbf{r}^{\prime}(s)\right|$ is the unit tangent vector; $A$ and $B$ are referred to as the bending and stretching modulus, respectively. One can further show that $A$ and $B$ are related via $A=(\pi / 4) E a^{4}, B=\pi E a^{2}$, where $E$ is the material's Young's modulus and $a$ is the radius of the rod $[9]$.

One important observation is the fact that polymers are usually embedded into a thermal environment and are therefore steadily hit by molecules with kinetic energy of the order $k_{\mathrm{B}} T[2$. Relating this thermal energy scale to the elastic energy of the 
Table 1.1.: Length scales concerning the bending elasticity of biopolymers.

\begin{tabular}{llll}
\hline Chain type & Chain length & Persistence length & $l_{0} / l_{\mathrm{p}}$ \\
& $l_{0} / \mathrm{nm}$ & $l_{\mathrm{p}} / \mathrm{nm}$ & \\
\hline DNA $\sqrt{10}$ & $10^{4}-10^{5}$ & $\sim 50$ & $10^{2}-10^{3}$ \\
Actin filament $\sqrt{11}$ & $\sim 10^{4}$ & $\sim 10^{3}$ & $10^{1}$ \\
Microtubule $[1]$ & $\sim 10^{3}$ & $\sim 10^{6}$ & $10^{-3}$ \\
\hline
\end{tabular}

rod, it turns out that, e.g., a $90^{\circ}$ bend into a quarter circle of radius $R$ costs an energy $\mathcal{E}_{\text {bend }}^{90^{\circ}}=\pi A /(4 R)\left[8\right.$. If we now define the persistence length $l_{\mathrm{p}}:=A /\left(k_{\mathrm{B}} T\right)$, then $\mathcal{E}_{\text {bend }}^{90^{\circ}} /\left(k_{\mathrm{B}} T\right)=\pi l_{\mathrm{p}} /(4 R)$. This shows that a polymer will randomly bend given that its contour length $l_{0}$ exceeds its persistence length $l_{\mathrm{p}}$ 8. Table 1.1 shows empirical values for the persistence and contour lengths of some important biopolymers. There is a wide spectrum of stiffnesses possible: A long DNA strand will naturally occur in a random coil state, whereas a suspended microtubule has a relatively well defined polymer axis. One usually categorizes into flexible $\left(l_{\mathrm{p}} / l_{0} \ll 1\right)$, semiflexible $\left(l_{\mathrm{p}} / l_{0} \sim 1\right)$ and stiff $\left(l_{\mathrm{p}} / l_{0} \gg 1\right)$ polymers. Note, however, that thermal bending fluctuations, in general, are significant even on length scales smaller than the persistence length [12].

In Appendices A.1 and A.2 we discuss the effect of thermally excited bending fluctuations in the context of statistical physics. In fact, we introduce the freelyjointed chain (FJC) model (Appendix A.1), which treats the polymer as a collection of uncorrelated elements with a fixed characteristic length (similar to the paper clip model shown in Fig. 1.1) and the wormlike chain (WLC) model 13 (Appendix A.2), which treats the polymer as an inextensible elastic rod (Eq. (1.1) without the stretching term). In a nutshell, we demonstrate that polymers possess a so-called entropic elasticity that acts against externally applied stretching forces. There are many more possibilities to realize a coiled up polymer configuration (large entropy) than a stretched out configuration (small entropy). So the force that is needed to hold the polymer ends at a certain distance reflects the competition between entropy and energy, which manifests itself in the fact that the system minimizes its free energy. Our discussion in Appendices A.1 and A.2 can be summarized in the following so-called force-extension relation for the WLC model [6]:

$$
f(u)=\frac{k_{\mathrm{B}} T}{l_{\mathrm{p}}}\left(\frac{u}{l_{0}}+\frac{1}{4\left(1-u / l_{0}\right)^{2}}-\frac{1}{4}\right),
$$




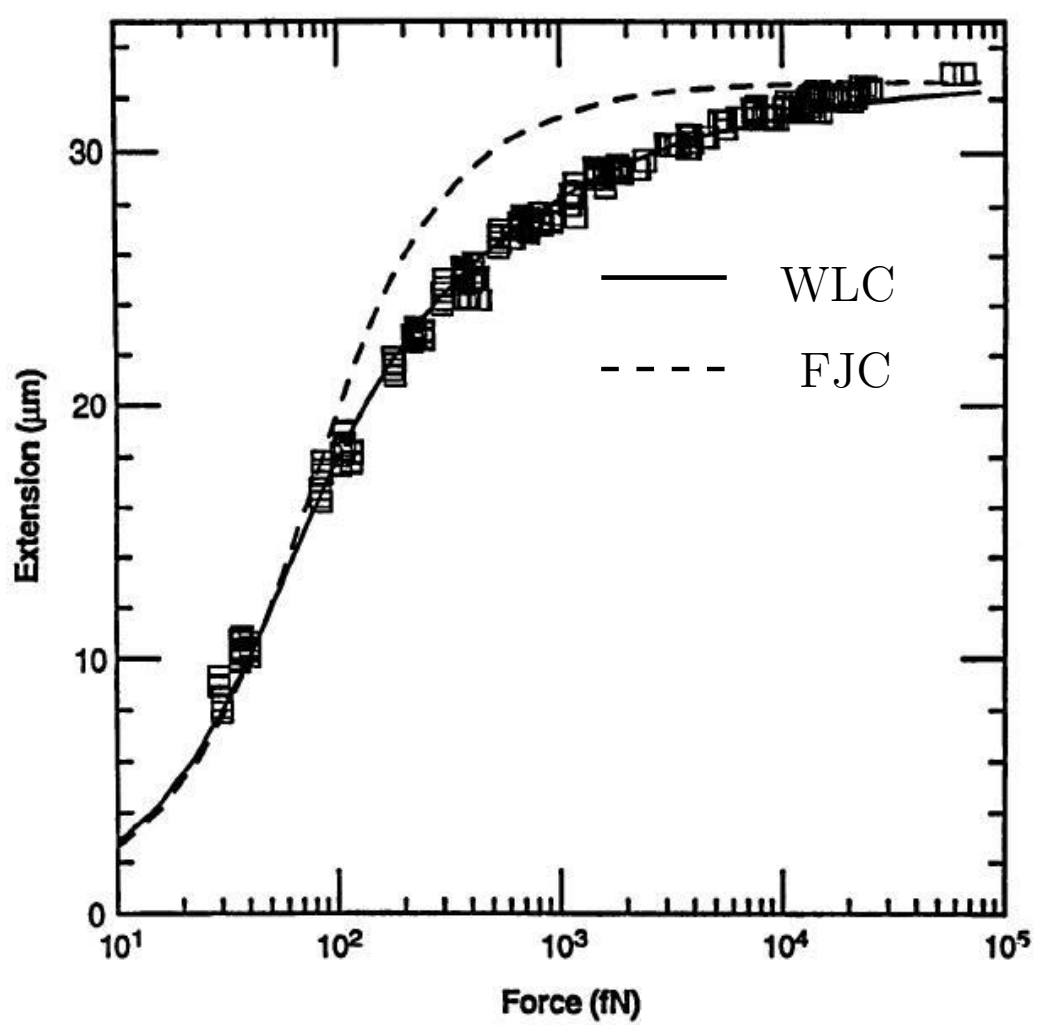

Figure 1.4.: Experimental force-extension data for DNA fitted by the numerical solution of the WLC model (solid curve). For comparison, the FJC model (dashed curve) is shown, with parameters chosen to fit the small extension data. The interpolation formula Eq. (1.2) comes very close to the numerical exact solution. Figure adapted from [14]. Reprinted with permission from AAAS.

where $f$ is the externally applied stretching force and $u$ is the end-to-end distance of the polymer. Equation (1.2) is an interpolation formula since there is no analytic solution to the WLC model known. However, Eq. (1.2) is asymptotically exact in the limit of small and large forces and does not differ from the numerical exact solution to the model by more than $16 \%[12$. Equation 1.2 is taylored to be applied for flexible polymers $\left(t_{0} / l_{\mathrm{p}} \gg 1\right)$ because there is no force at zero extension and the linear spring constant is that of the FJC, i.e., $k_{\mathrm{FJC}}=3 k_{\mathrm{B}} T /\left(2 l_{0} l_{\mathrm{p}}\right)=\partial f /\left.\partial u\right|_{u=0}$ (see Eqs. A.7) and (A.26). Single molecule experiments have shown that Eq. 1.2 captures the stretching response of, e.g., DNA 14 very well (see Fig. 1.4). Similarly, one can write interpolation formulas for semiflexble or stiff polymers, which have the same functional form, but realize a finite rest-length at zero force and have a different linear spring constant, i.e., $k_{\text {stiff }}=90 A^{2} /\left(k_{\mathrm{B}} T l_{0}^{4}\right)$ (for both see Eq. (A.24)). 


\section{Introduction}

A closed-form expression for the force-extension relation, like Eq. (1.2), is very practical for the implementation in the context of numerical simulations, as well as for analytical theories (see, e.g., [15, 16]). The characteristic features of Eq. (1.2) are the linear force response for small extensions and the characteristic divergence of force as $u \rightarrow l_{0}$, with $f(u) \sim\left(1-u / l_{0}\right)^{-2}$. Consequently, the elastic response of a polymer in the WLC model is, in general, nonlinear; it shows pronounced stiffening under longitudinal load, which can be characterized via the so-called differential stiffness, $k_{\mathrm{WLC}}(u):=(\partial f / \partial u)(u)$, with

$$
k_{\mathrm{WLC}} \sim\left(1-u / l_{0}\right)^{-3} \sim f^{3 / 2}, \quad \text { as } u \rightarrow l_{0}
$$

The scaling relation above is a characteristic feature that is important in the context of polymer networks as well.

Of course, any real polymer will not resist arbitrarily large forces - at some point it will rupture and, even before that, stretch along its backbone (energy contribution from the second term in Eq. (1.1)). Models that take into account the enthalpic stretching contribution can be found in, e.g., 77, 17]. Fig. 1.5 shows the differential stiffness of the model presented in [17] together with the asymptotic (large force) response of the WLC model.

The mechanical properties of individual polymers are crucial for the study of polymer networks. In particular, it is the nonlinearity in the response to external stretching that has significant effects on the asymptotic network elasticity and structure.

\subsection{Biopolymer networks}

We now turn to collections of biopolymers embedded into an aqueous solution. In the cell, this solution is called the cytosol and consists of $\sim 70 \%$ water 18. Depending on concentration and length, polymer assemblies can form dilute or entangled solutions, where polymers have no permanent interactions [19]. Adding crosslinking agents that form, possibly permanent, bonds between filaments gives rise to what we call a crosslinked polymer network (see Fig. 1.6) that, together with the solvent, forms a polymer gel [2]. In the biological context, crosslinkers are usually proteins, which bind non-covalently to their corresponding biopolymers. They can be either mostly rigid, i.e., not show significant compliance before rupturing, or flexible, meaning 


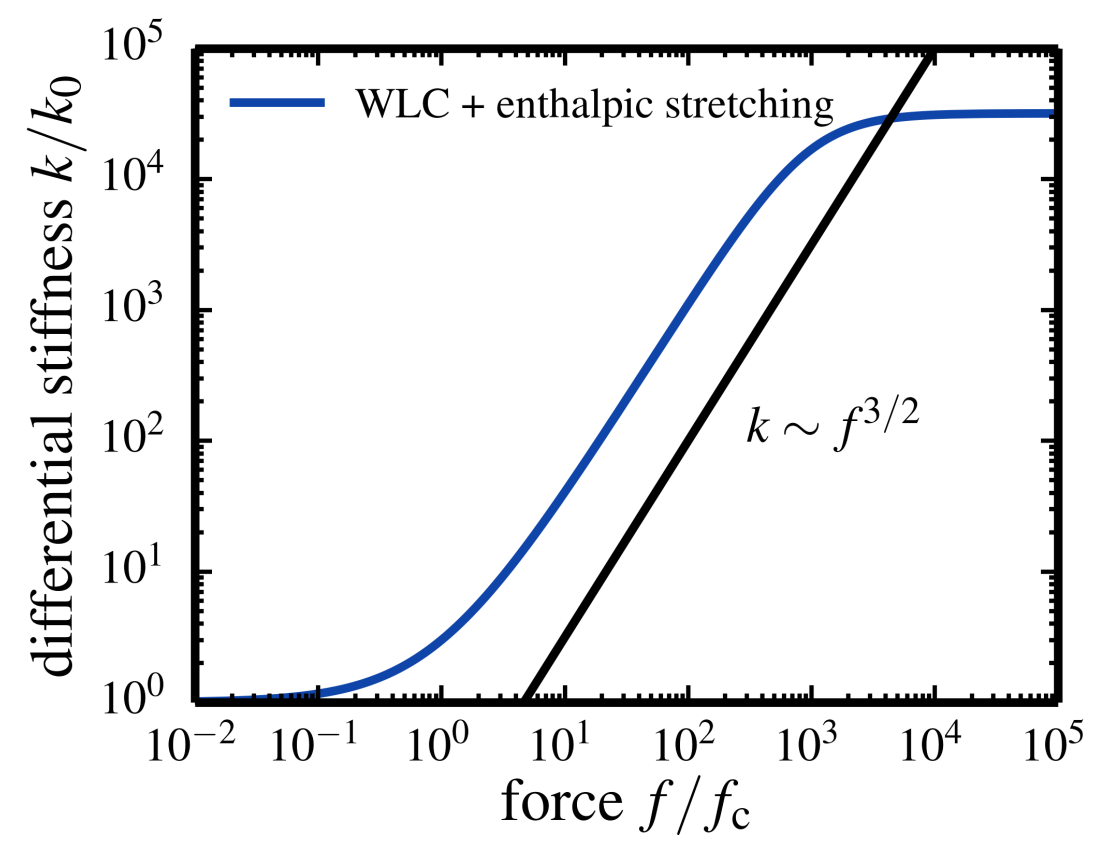

Figure 1.5.: Differential stiffness $k=d f / d u$ of an extensible wormlike chain (based on Eq. 35 in [17]), normalized by the linear stiffness $k_{0}$, as a function of force $f$, normalized by the critical force $f_{\mathrm{c}}$, at which the stiffness has increased by a factor of three. The black solid line shows the large force scaling, $k \sim f^{3 / 2}$, of an inextensible wormlike chain.

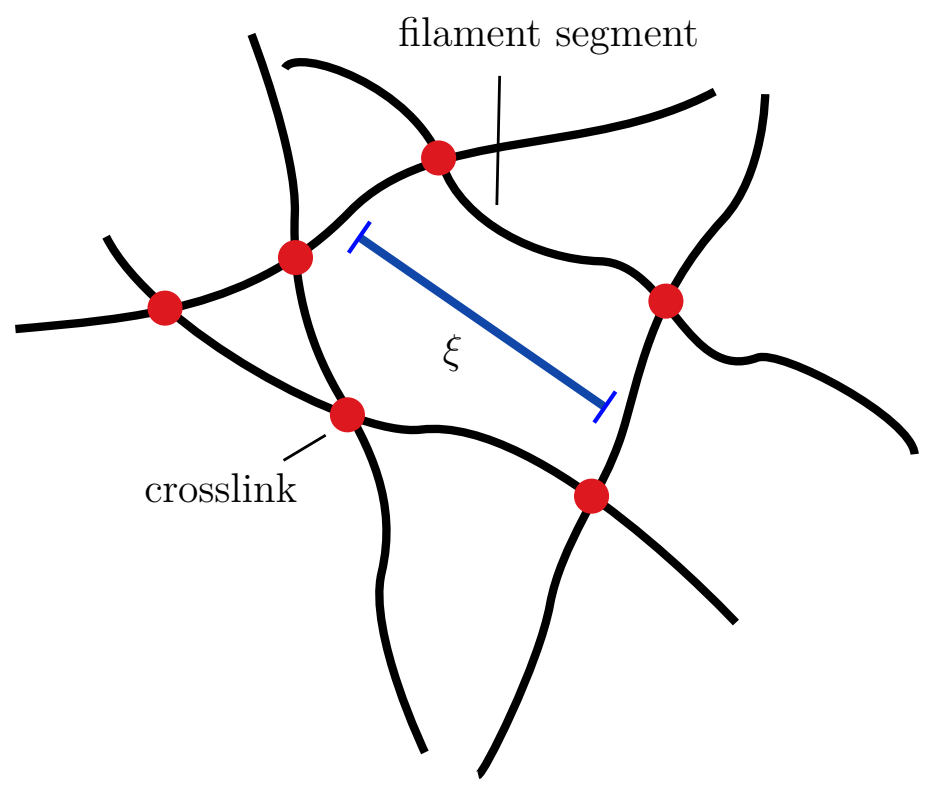

Figure 1.6.: Sketch of a network of semiflexible polymers connected by rigid pointlike crosslinks, with average mesh size $\xi$. 


\section{Introduction}

that they can contribute significantly to the network compliance. There even exist so-called molecular motors, which - apart from crosslinking biopolymers - are further able to actively generate forces under consumption of ATP, the energy currency of the cell [1].

Depending on the assembly dynamics and the properties of the involved constituents and their relative concentrations, biopolymer networks show a variety of complex architectures. We are interested in the mechanical properties of these complex, usually highly dynamic assemblies. The field of study that deals with the material properties of soft matter is commonly called rheology. It deals with the viscoelastic properties of complex materials. The term "viscoelastic" emphasizes the fact that the mechanical response, of, e.g., a polymer gel, is characterized by a viscous contribution due to the solvent as well as an elastic part from the polymer network.

A common - and quite general - scheme, which is used to gain understanding about the collective properties of complex matter, is a combination of experimental studies and modeling approaches. We first mention some experimental results relevant for our later discussions followed by an overview of different modeling techniques that are present in the field.

\subsubsection{Experimental studies}

There is a variety of techniques that can be applied to quantitatively study the viscoelastic properties of polymer gels (see, e.g., [20]). Here, we focus on bulk rheology measurements, where one uses a device called a rheometer (see Fig. 1.7). The sample is located in between two plates, where the upper plate can rotate. Thereby, it is possible to externally apply a shear deformation, which is potentially oscillatory in nature, with a certain frequency. By measuring the torque on the upper plate one can then relate shear stress and shear strain as a function of time, giving access to quantities like the shear modulus, which has a viscous and an elastic part. Here we focus on the elastic response of the material, i.e., the zero frequency or quasistatic limit, where all stresses due to the viscous response can relax. The elastic shear modulus $G$ assumes a linear relation between shear stress $\sigma$ and strain $\gamma$, in particular, $\sigma=G \gamma$.

However, we have already noticed that single biopolymers have a nonlinear forceextension relation; hence we expect biopolymer networks to stiffen under strain, too. There exist several experimental techniques that are able to capture this nonlinear elastic response. Here we present a few, which are all based on bulk rheometry: (i) 


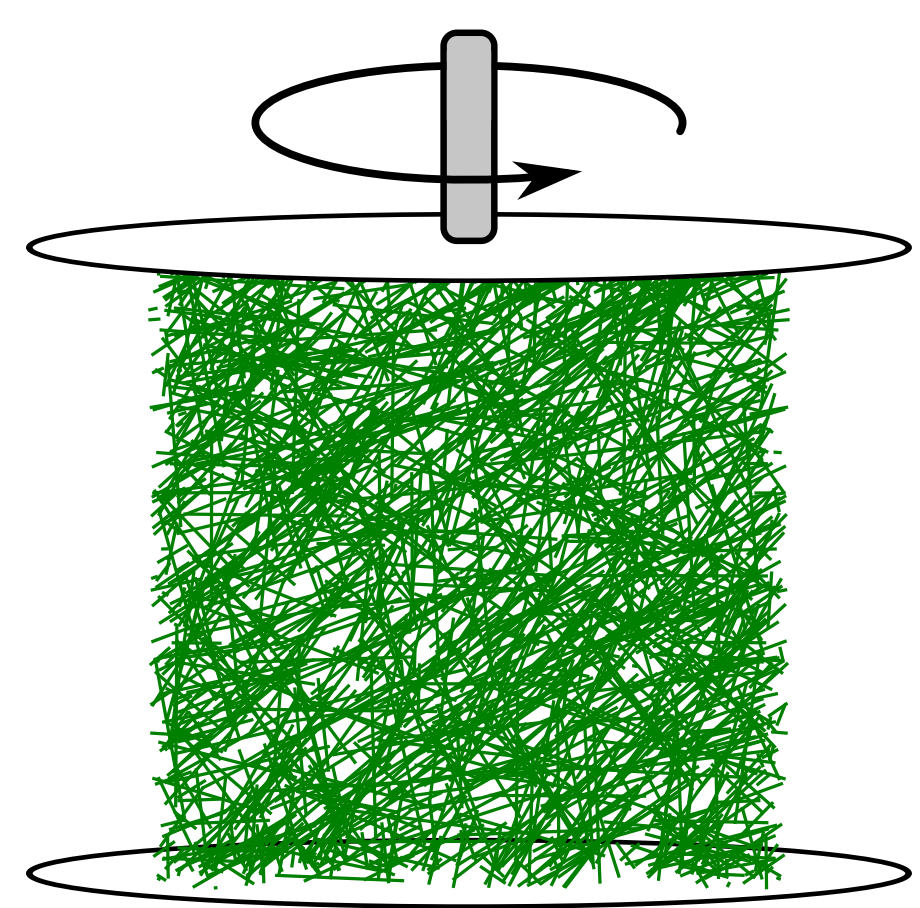

Figure 1.7.: Bulk rheology: A polymer network (shown in green) is sheared between two plates of a rheometer. The upper plate usually rotates in an oscillatory fashion.

LAOS (large amplitude oscillatory shear) experiments take into account higher order contributions to the shear stress via a Fourier decomposition of the oscillatory signals 21 23]; (ii) Strain ramp measurements increase the applied shear strain at a small rate (without oscillating) and measure the (possibly nonlinear) increase in stress 22 . 24. 25; (iii) The prestress method applies a constant stress $\sigma_{0}$ in order to bring the network into a nonlinear regime. Then a small oscillatory stress is superimposed, which, ideally, results in an elastic response that is now linear and can be analyzed by the usual procedure 24, 26,31].

Figure 1.8 shows an example of an experimental result for the nonlinear elastic response of biopolymer networks obtained by prestress measurements. The shown quantity is the differential elastic modulus, $K^{\prime}:=d \sigma_{0} / d \gamma$, which is equal to the conventional elastic shear modulus given that the stress-strain relationship is linear. One can clearly see, that the networks show pronounced stiffening behavior, i.e., $K^{\prime}$ increases drastically with the applied prestress $\sigma_{0}$. This is not surprising since we already saw in Section 1.1 that individual biopolymers stiffen under extensional load. Since Fig. 1.8 is double-logarithmic, it reveals the characteristic scaling properties of the modulus with stress. In particular, there is a regime with $K^{\prime} \sim \sigma_{0}^{3 / 2}$, which is the same scaling as has been observed for the differential stiffness of single biopolymers 


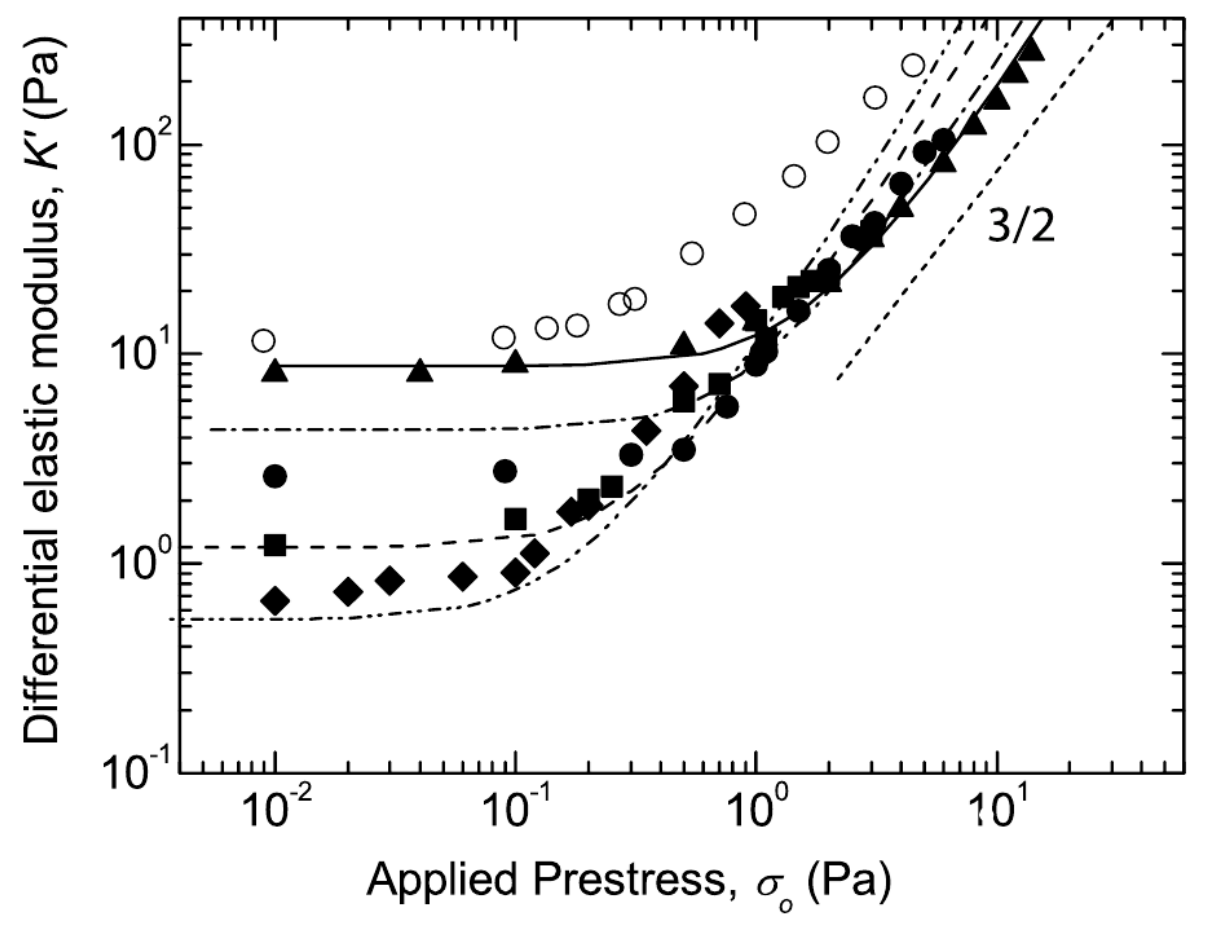

Figure 1.8.: Differential elastic modulus $K^{\prime}$ as a function of shear stress $\sigma_{0}$ for actin networks irreversibly crosslinked with the noncompliant crosslinker scruin. The symbols correspond to different actin concentrations and crosslink densities. The dashed line indicates power law scaling $K^{\prime} \sim \sigma_{0}^{3 / 2}$. Adapted from [31]. Reprinted with permission from AAAS. 
(see Eq. (1.3)). This observation suggests that one can explain the network's elasticity with the help of the individual polymer response.

At this point, modeling comes into play. Experimentally, one can relate stress and strain in order to obtain bulk properties like the shear modulus. These measurements, however, correspond to averages, i.e., measure the average shear stress over the whole sample - or, equivalently, the amount of elastic energy that is introduced due to the shear deformation. Bulk elastic measurements do not determine how stresses are distributed within the network locally, e.g., which filaments carry most of the energy and in which deformation mode.

\subsubsection{Modeling strategies}

One important task of a model is to capture prominent features of experimentally observed phenomena based on simplifying assumptions about the system under consideration. Ideally, a simple model is equipped with a few parameters allowing for a comprehensive understanding of the physics in different regimes. A model that is as close to reality as possible may potentially be able to capture most of the experimentally observed features. However, it can be difficult to grasp the physical mechanisms that underly a certain observation. In particular, very complex models often lack an analytical approach and are potentially more challenging in terms of simulations. Note further that the fact that a specific model is able to reproduce a certain experimental observation does not prove that it describes the correct physics. It must be internally consistent and should provide further predictions that can be tested in experiment, in order to provide more evidence for its applicability.

Here, we want to distinguish between two classes of models for polymer networks that follow different simplification approaches: (i) Continuum models, which often define a so-called elementary or unit cell and then derive a continuum formulation of the problem, and (ii) Network models, which take into account the connectivity, i.e., topology, of the particular polymer network.

For reviews on state of the art modeling techniques for polymer networks see, for example, 12,32 .

\section{Continuum models}

Continuum models usually take into account the force-extension relation of the individual polymers but not the network topology, i.e., the mechanical coupling 


\section{Introduction}

between the crosslinked polymer strands in the network. Instead of solving for force balance at each node in the network, one assumes that one can treat the individual fibers or fractions of the network on a certain length scale, e.g., the network's mesh size (see Fig. 1.6), as independent of each other.

Storm et al., for example, average over all possible filament orientations while assuming that every filament deforms affinely [33], i.e., follows the externally applied simple shear deformation. Under these assumptions, this so-called affine model allows for the calculation of the elastic energy in the network as a function of deformation and hence the bulk elastic properties, like the shear modulus.

Other approaches define a unit or elementary cell (see, e.g., [34, 35]), which consists of several polymer chains; this cell is then repeated in order to form the effective network. Based on the deformation modes assigned to the unit cell elements one can use homogenization techniques to derive an expression for a continuous strain energy density. With this at hand, one has access to the elastic response of this effective material under various deformations and geometries via the framework of continuum mechanics.

We have seen that continuum models do not take into account network topology. Moreover, they may assume that the network deforms affinely or treat the network as homogeneous above a certain length scale. Although these models do capture essential bulk elastic properties of various types of polymer networks [15, 16, 34], they lack the capability of describing long range correlations between filaments or stress localization along paths at large deformations - especially when the nonlinear response (see Eq. 1.2p) of the filaments comes into play.

\section{Network models}

Network models take into consideration the network structure explicitly. A crosslinked network is represented as a collection of nodes that are connected via polymer segments (see, e.g., Fig. 1.9). One assigns an elastic energy based on the degrees of freedom, i.e., the node positions. The elastic properties can be probed by application of quasistatic deformations. One starts with a system in force balance (energy minimum), applies a small deformation (e.g., simple shear), so that the node forces are imbalanced, and minimizes the energy via an appropriate algorithm. By continuing this procedure one gains access to the system's elastic energy as a function of applied deformation - so the bulk elastic response of the network - as well as local properties like node displacements or forces in individual polymer segments. 

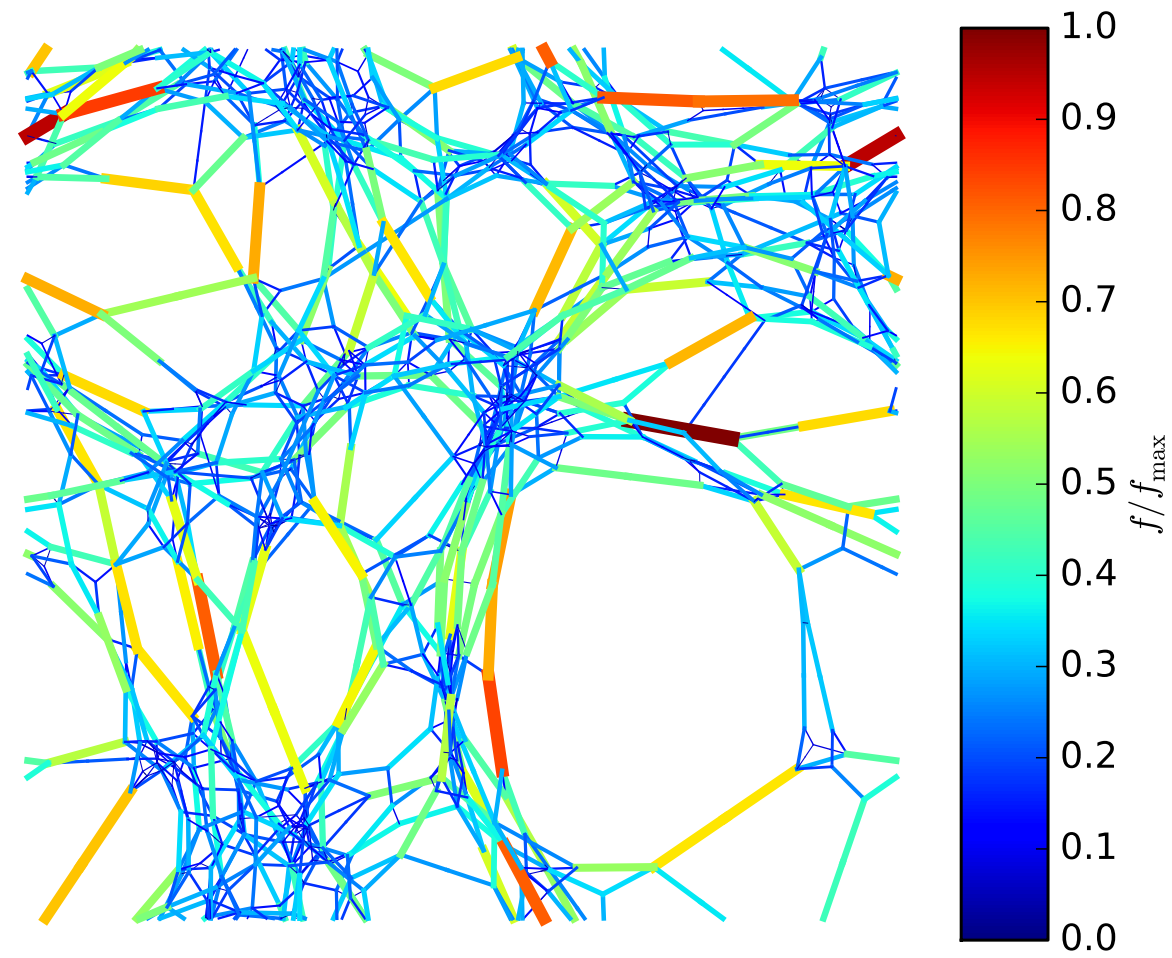

Figure 1.9.: Example of a 2D wormlike chain network model, with the tensional forces color coded and highlighted via line thickness. Periodic boundary conditions are applied.

One can differentiate between various network models by looking at which deformation modes of the individual filaments are taken into account. A commonly used framework includes bending as well as stretching degrees of freedom, with quadratic energy terms, i.e., without the nonlinear response to stretching for large deformations. The initial network for 2-dimensional (2D) simulations is often constructed as a (disordered) mikado network (see Fig. 1.7), meaning that one starts with a certain number of filaments of a given length and crosslinks at the intersection sites. One can then study which deformation modes (bending or stretching) are excited upon the application of, e.g., a simple shear deformation - depending on crosslink density or filament length 36 39]. Others have extended this approach to 3-dimensional (3D), mostly lattice-based, systems (see, e.g., [40]), or further took into account the full nonlinear force-extension relation of the polymer segments [41, 42] leading to strongly heterogenous elastic properties 42 . Moreover, composite systems consisting of polymers of varying stiffnesses have been introduced [15, 43]. 


\subsection{Outline of the thesis}

This thesis is divided into two parts, which deal with different types of polymer networks. In both, we perform full network simulations as well as analytical modeling.

Chapter 2 deals with composite networks of rigid rods connected by flexible crosslinks, for which we take into account the full nonlinear force-extension relation. We perform quasistatic simulations in order to characterize the bulk elastic properties as well as the pronounced heterogeneous force distributions within these networks. Additionally, we develop a single filament model, which is based on affine deformations.

In Chapter 3, we analyze random spring networks on the unit circle. We develop a simulation framework that guarantees conservation of network topology when simulating on periodic structures, e.g., when periodic boundary conditions are applied. Furthermore, we provide an analytical theory that provides force distributions in these networks by taking into account the entire network topology. It is based on probability theory and graph theoretical considerations. 


\section{Networks with rigid filaments and compliant crosslinks}

This chapter contains the results from the publication:

- K. M. Heidemann, A. Sharma, F. Rehfeldt, C. F. Schmidt \& M. Wardetzky. Elasticity of 3D networks with rigid filaments and compliant crosslinks. Soft Matter 11, 343-354 (2015)

We give a short summary of the key findings obtained within the corresponding study, before presenting the full article.

\subsection{Key results}

In this study, we consider composite networks composed of rigid rods connected by flexible crosslinks. In particular, the rods are considered to be perfectly rigid, i.e., do not carry any elastic energy, and the crosslinks are modeled via the nonlinear forceextension relation of a flexible wormlike chain (see Eq. (1.2)). In this model system, the elastic response is due to the crosslinks, whereas the rods act as scaffolding agents. We carry out 3D simulations (see Fig. 2.1) and characterize the network response under quasistatic simple shear deformations. Moreover, we develop an affine theory that considers a single representative rod - decorated with crosslinks - and averages over all possible rod orientations in order to obtain the total elastic energy in the system (see Fig. 2.2 for a schematic comparison of the two approaches).

By combining these two modeling strategies, we are in the comfortable situation of having access to the entire modeling spectrum discussed in Section 1.2.2. On the one hand, a full network model that considers the actual topology of the network and therefore accounts for the discrete and disordered nature of the polymer network, as well as the correlations between the filaments during deformation. On the other hand, a simplified continuum model that allows for an analytical treatment of the problem 


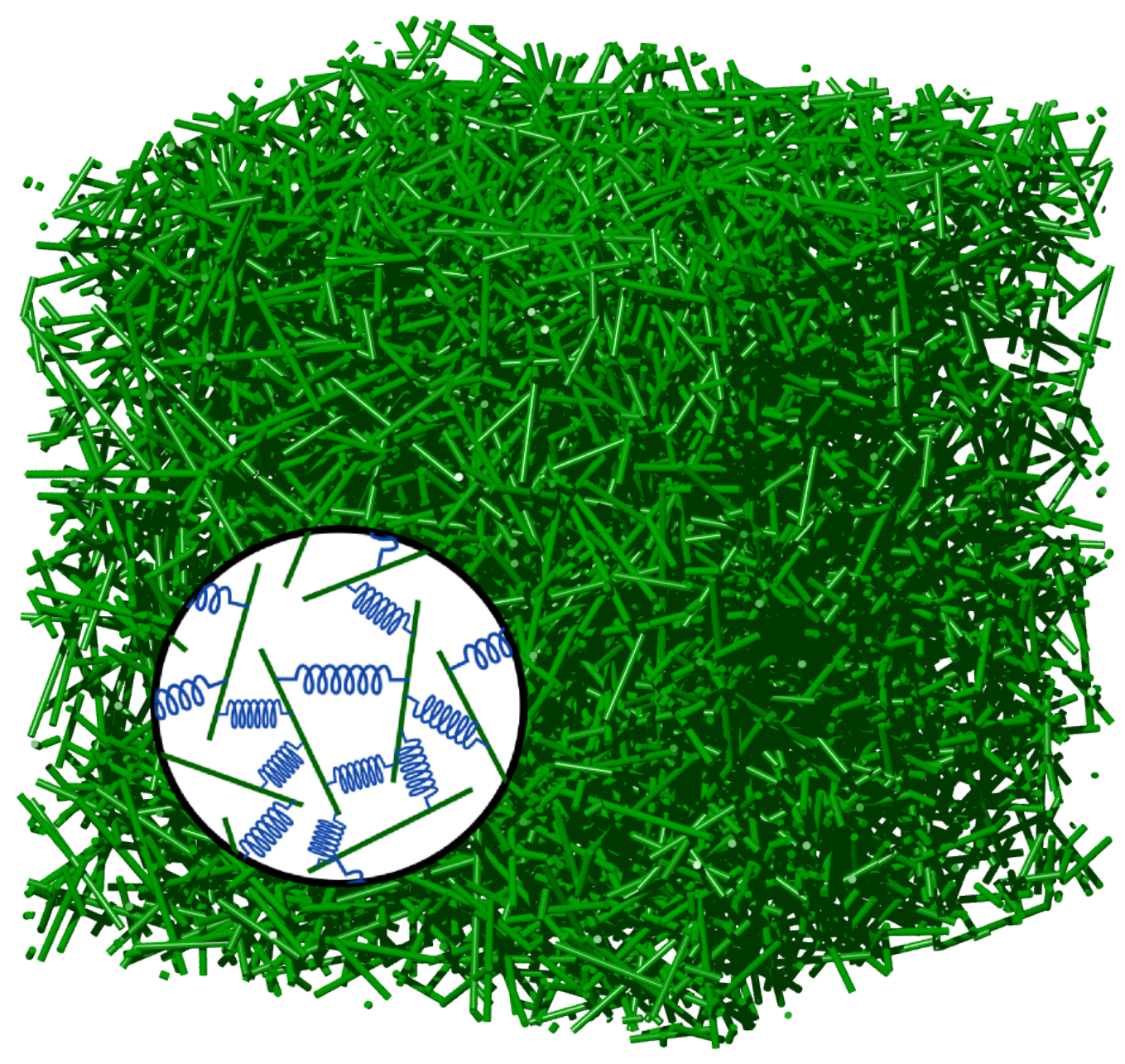

Figure 2.1.: Snapshot of the 3D simulation in the undeformed state. Rigid rods are shown in green, crosslinks have been omitted, but are shown in the (zoomed in) schematic as blue (nonlinear) springs. We further apply periodic boundary conditions in all spatial dimensions. 
(a)

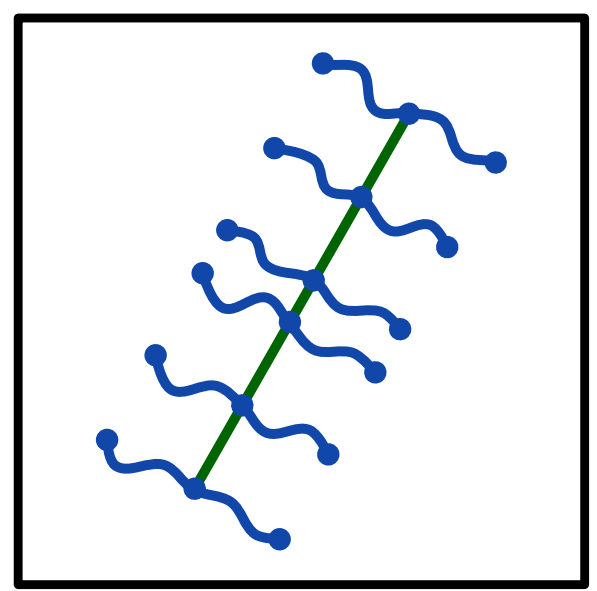

(b)

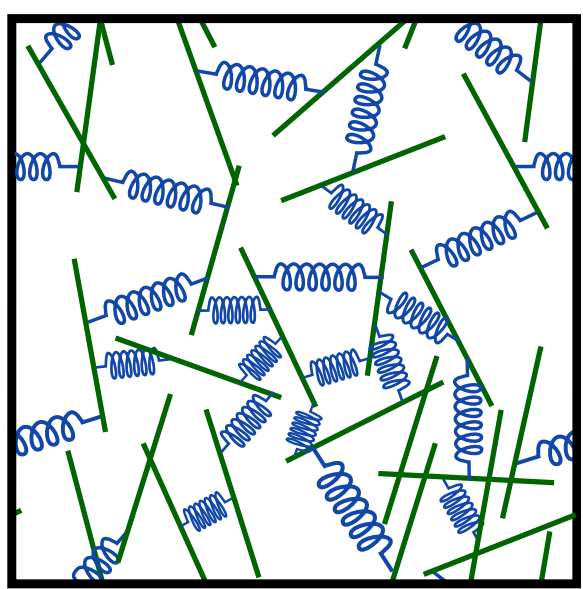

Figure 2.2.: (a) Affine theory: The network is represented by a single rigid rod (green) with flexible crosslinks (blue). (b) Simulations: The network topology is explicitly taken into account by connecting a collection of rigid rods (green) via flexible crosslinks (blue springs). Note that we apply periodic boundary conditions.

and thereby enables us to get further understanding of some of the fundamental mechanisms that might determine the elastic response of the model system under consideration. We can compare both approaches and thereby check which predictions from the analytical theory can indeed be observed in the network simulations - and, likewise, identify network phenomena that cannot be captured by the continuum model.

In the context of the affine theory, we first consider the continuum limit, which assumes a continuous distribution of crosslinks along a single rod, as well as a continuous distribution of rod orientations in the system. Note further, that there must be a maximum strain $\gamma_{\mathrm{d}}$ because the crosslinks have finite contour length and do not rupture, i.e., respond with an infinite force at finite extension (see Eq. (1.2)). We can derive analytical expressions for the total elastic energy in the large strain limit, $\gamma \rightarrow \gamma_{\mathrm{d}}$. In Table 2.1, we summarize the analytical results. First of all, we notice that the divergence of stress $\sigma$ with strain $\gamma$ becomes weaker with an increase in dimensionality of the system. This is rationalized by the fact that an increased amount of orientational averaging - as happens for higher dimensions - leads to smaller fractions of crosslinks that are close to maximum extension and therefore dominate the stiffening of the network. However, the dependence of the differential modulus $K$ on stress $\sigma$ becomes stronger; surprisingly, we find exponential stiffening of the modulus with stress in three dimensions. 
Table 2.1.: Asymptotic scaling behavior of the bulk elastic quantities - shear stress $\sigma$ and differential elastic shear modulus $K$-as the shear strain $\gamma$ approaches its maximum value $\gamma_{d}$. The scaling behavior depends on the dimensionality (Dim.) of the system.

\begin{tabular}{clll}
\hline $\operatorname{Dim}$. & $\sigma(\gamma)$ & $K(\gamma)$ & $K(\sigma)$ \\
\hline 1D & $\sigma \sim\left(1-1 / \gamma_{\mathrm{d}}\right)^{-1}$ & $K \sim\left(1-1 / \gamma_{\mathrm{d}}\right)^{-2}$ & $K \sim \sigma^{2}$ \\
2D & $\sigma \sim\left(1-1 / \gamma_{\mathrm{d}}\right)^{-1 / 2}$ & $K \sim\left(1-1 / \gamma_{\mathrm{d}}\right)^{-3 / 2}$ & $K \sim \sigma^{3}$ \\
3D & $\sigma \sim-\log \left(1-1 / \gamma_{\mathrm{d}}\right)$ & $K \sim\left(1-1 / \gamma_{\mathrm{d}}\right)^{-1}$ & $K \sim e^{c \sigma}$ \\
\hline
\end{tabular}

Taking into account the discrete nature of the network, i.e., noticing that there is only a finite number of crosslinks per rod as well as a finite number of rod orientations present in the network, we find numerically (replacing integrals by finite sums) that the only asymptotic scaling behavior is that of the single wormlike chain $\left(K \sim \sigma^{3 / 2}\right)$-independent of the dimensionality of the system (see Fig. 2.3. . Still, the stronger dependence of the modulus on stress can be observed for intermediate stresses (see Fig. 2.3. and Fig. 2.6 for more details). There is an intuitive explanation for the breaking down of the continuum theory: Due to the divergence of the crosslink force-extension relation any calculation of the energy via integration, i.e., orientational averaging and integrating along the backbone of the rod (see Section 2.2.2), will eventually fail since individual rods/crosslinks contribute an arbitrarily larger amount of energy than others. Therefore, the integrals do not represent the true elastic energy of any finite system for large deformations.

The bulk elasticity results of our 3D network simulations are in good agreement with the affine theory. We observe the asymptotic power law scaling of the modulus with stress with an exponent $3 / 2$ and a stronger dependence on stress in the intermediatestress regime (see Fig. 2.3). However, our results are in stark contrast to a study by Broedersz et al. 44, 45], which inspired the assumptions in our model system, but considers an additional effective medium that is coupled to the single rod (like two springs in series). Their model results in a linear scaling $K \sim \sigma$ independent of the dimensionality of the system. We argue that this result is inconsistent with the model itself since it suggests that one can apply arbitrarily large strains without a divergence in stress $\left(\sigma \sim e^{b \gamma}\right)$. This, however, contradicts the model assumption that crosslinks and rods have a finite length, i.e., have a diverging force-extension relation. 


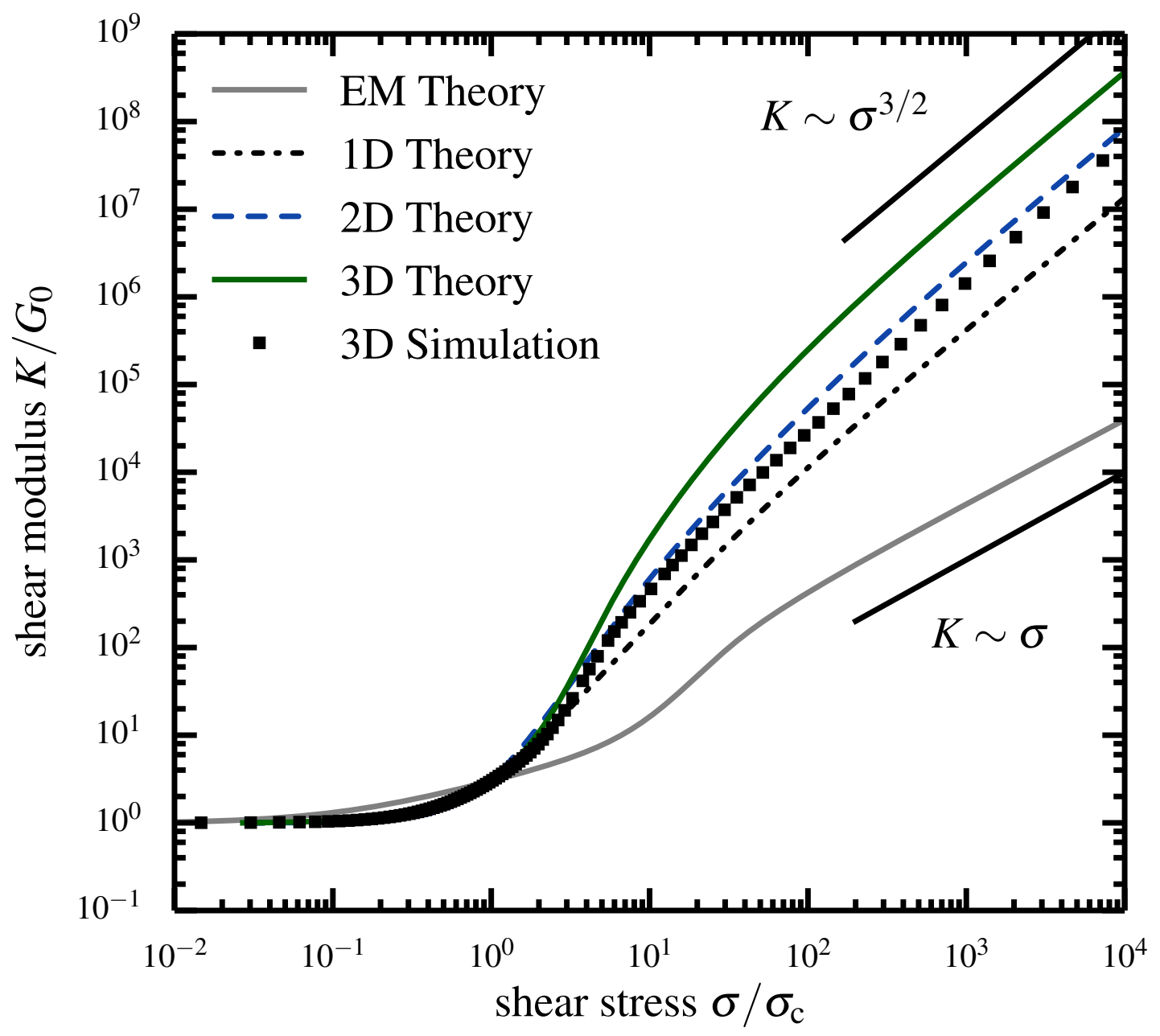

Figure 2.3.: Differential modulus $K=d \sigma / d \gamma$ as a function of shear stress $\sigma$, rescaled by the linear elastic modulus $G_{0}:=\left.K\right|_{\gamma=0}$ and critical stress $\sigma_{\mathrm{c}}:=\sigma\left(\gamma_{\mathrm{c}}\right)$, respectively, where $\gamma_{\mathrm{c}}$ is defined via $K\left(\gamma_{\mathrm{c}}\right)=3 G_{0}$. The effective medium ("EM Theory") result is that of Broedersz et al. 44, 45. The other three theoretical results ("1D/2D/3D Theory") correspond to a numerical evaluation of our affine theory for a finite crosslink density (number of crosslinks per rod) of 60 . The simulation result has been obtained for a system of 3000 rods with crosslink density 60 . 
Lastly, we consider an aspect that can only be captured by our network simulations that take into account network topology explicitly. We have already noticed that the asymptotic network response is dominated by individual WLC crosslinks. However, we did not consider the distribution of forces within the network, which we can access in our simulations. A striking feature is the emergence of, what is commonly called, force chains $42,46,48]$. Any network will eventually develop a system spanning stretched out path of rods and crosslinks that carries an exceedingly large fraction of the system's total energy and therefore dominates the elastic response (see Fig. 2.4). The nonlinear crosslink force-extension relation leads to more and more energy being accumulated; at the same time, the remaining part of the network cannot reduce the amount of stretch in the force chain, since additional undulations in the path would only further increase its length. The fact that the transition to $K \sim \sigma^{3 / 2}$ scaling happens at smaller stresses in the $3 \mathrm{D}$ simulations than in the $3 \mathrm{D}$ affine theory (see Fig. 2.3 can be partly attributed to the emergence of force chains, since such a stretched out path leads to single WLC scaling at, possibly, smaller stresses than in the affine theory, which does not consider network topology, i.e., force chains.

Localization of large forces in individual chains can be particularly crucial when it comes to network remodeling. Presumably, crosslinks within the force chains rupture or unbind first and thereby might give rise to large rearrangements. It would thus be interesting to analyze the plastic response of nonlinear polymer networks in a future study. 


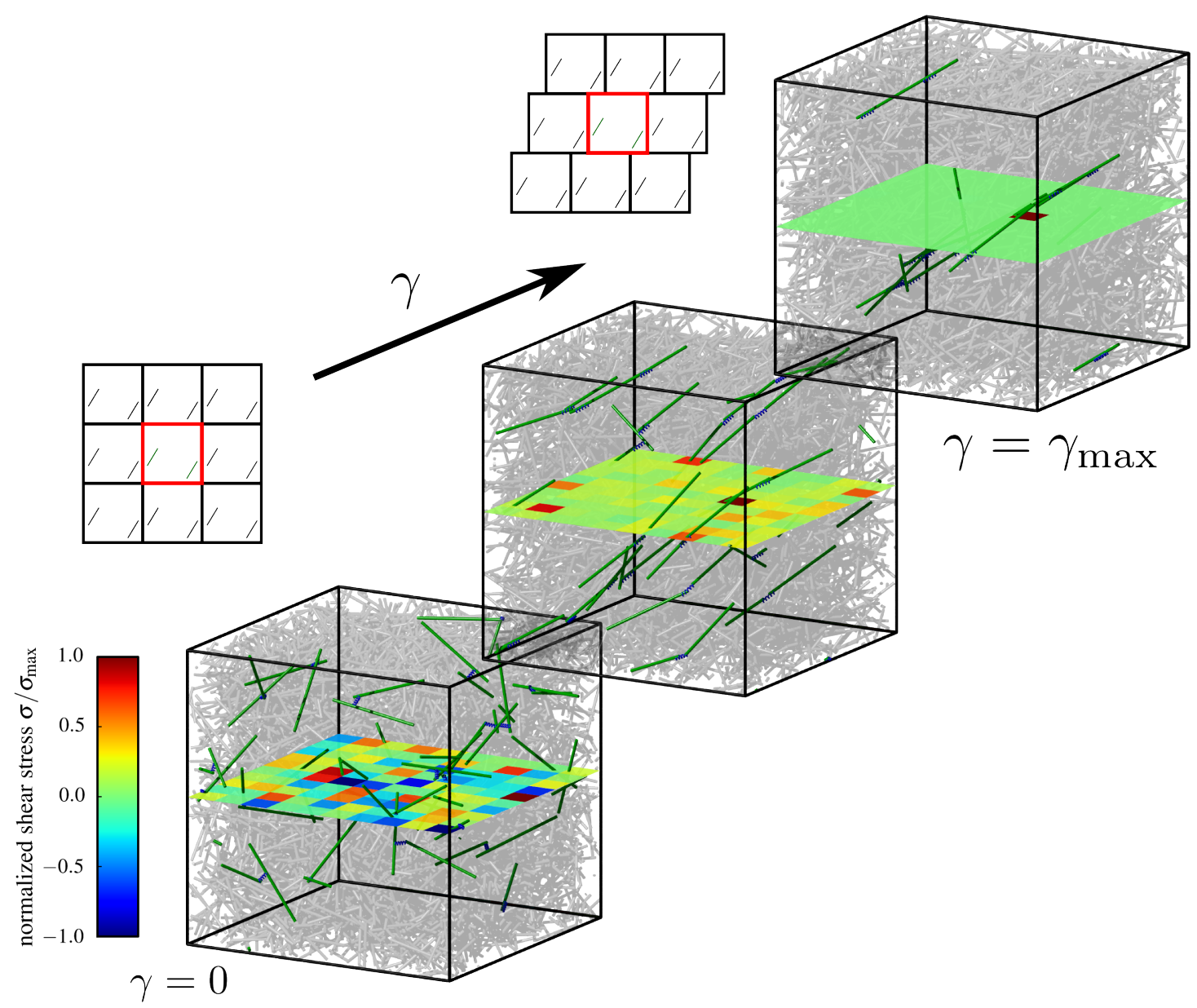

Figure 2.4.: Emergence of a force chain: 3D simulation with the 20 most stretched crosslinks and the corresponding filaments highlighted in color, at increasing shear strain (from left to right). The schematics on the top visualize Lees-Edwards boundary conditions [49], which are used for shearing with periodic boundary conditions. Furthermore, the shear stress is mapped for one plane, showing that it is eventually dominated by the force chain. 


\title{
2.2. Publication
}

\begin{abstract}
Disordered filamentous networks with compliant crosslinks exhibit a low linear elastic shear modulus at small strains, but stiffen dramatically at high strains. Experiments have shown that the elastic modulus can increase by up to three orders of magnitude while the networks withstand relatively large stresses without rupturing. Here, we perform an analytical and numerical study on model networks in three dimensions. Our model consists of a collection of randomly oriented rigid filaments connected by flexible crosslinks that are modeled as wormlike chains. Due to zero probability of filament intersection in three dimensions, our model networks are by construction prestressed in terms of initial tension in the crosslinks. We demonstrate how the linear elastic modulus can be related to the prestress in these networks. Under the assumption of affine deformations in the limit of infinite crosslink density, we show analytically that the nonlinear elastic regime in 1- and 2-dimensional networks is characterized by power-law scaling of the elastic modulus with the stress. In contrast, 3-dimensional networks show an exponential dependence of the modulus on stress. Independent of dimensionality, if the crosslink density is finite, we show that the only persistent scaling exponent is that of the single wormlike chain. We further show that there is no qualitative change in the stiffening behavior of filamentous networks even if the filaments are bending-compliant. Consequently, unlike suggested in prior work, the model system studied here cannot provide an explanation for the experimentally observed linear scaling of the modulus with the stress in filamentous networks.
\end{abstract}

\subsubsection{Introduction}

The mechanical properties of biological cells are governed by the cytoskeleton, a viscoelastic composite consisting of three main types of linear protein polymers: actin, microtubules, and intermediate filaments. These filamentous polymers are crosslinked by various binding proteins and constitute a dynamic complex network that maintains the structural integrity of the cell with the capacity for dynamic reorganization needed for active processes. Many in vitro studies have focused on reconstituted networks with rigid crosslinks $[1,12$. In the cytoskeleton, however, many of the crosslinks are themselves extended and highly compliant proteins. Such flexible crosslinks can strongly affect the macroscopic network elasticity [13 21]. Indeed, experimental 
studies show that composite networks can have a linear modulus as low as $\sim 1 \mathrm{~Pa}$, while being able to stiffen by up to a factor of 1000 [11, 14.

Here we analyze 3-dimensional (3D) composite networks theoretically, and we offer physical simulations thereof. Our networks are composed of randomly oriented rigid filaments that are connected by highly flexible crosslinks, each of which is modeled as a wormlike chain (WLC) [22, 23], which has been shown to accurately describe flexible crosslinkers, such as filamin 24,25$]$. In our approach we assume that the filaments are much more rigid than the crosslinks, meaning that the network elasticity is dominated by the entropic stretching resistance of the crosslinks.

In our theoretical analysis we adopt the widely employed assumption of affine deformations 16, 19, 26]. Under this premise, the network is assumed to deform affinely on the length scale of the filaments, which in turn is assumed to be much longer than the contour length of the crosslinks. Using a single filament description in the limit of a continuous distribution of crosslinks along the filament, we obtain the asymptotic scaling behavior of the elastic modulus with the stress in the nonlinear regime. We show that in 1-dimensional (1D) networks, the elastic modulus scales with the second power of the stress, whereas it scales with the third power in 2dimensional (2D) networks. Remarkably, there is no power law scaling in 3D - in fact, the elastic modulus of a 3D composite network increases exponentially with the stress. Numerical evaluation of the affine theory at finite crosslink densities - as opposed to a continuous distribution of crosslinks - shows that (i) the only asymptotic scaling is that of the modulus scaling with an exponent $3 / 2$ with the stress and that (ii) the dependence on dimensionality of the system is limited to an intermediate-stress regime. These findings are in agreement with our extensive physical simulations of 3D composite networks. For all cases, the elastic modulus diverges at a finite strain.

Our theoretical analysis is inspired by the mean-field model proposed by Broedersz et al. [16, 26]. In sharp contrast to our theoretical analysis and to the results of our physical simulations, however, these authors predict linear scaling of the elastic modulus with applied stress. In particular, for any finite strain, the elastic modulus remains finite in their model. While this linear scaling of the elastic modulus is in accordance with what has been observed experimentally [13, 20, 21], we here argue that this model does not adequately capture the elastic response of networks with rigid filaments and permanent (i.e., non rupturing or rebinding) crosslinks of finite length. 
In Ref. [19], the authors ruled out that the experimentally observed approximate linear scaling of the modulus with the stress might be due to enthalpic (linear) stretching compliance of the crosslinks or filaments. Here, we complement their analysis by physical simulations that take into account bending of filaments. Our results empirically show that the inclusion of bending rigidity does not impact the nonlinear stiffening behavior of composite networks either. We therefore conclude that the theoretical explanation for the linear scaling of the modulus with stress in experiments remains an challenging open problem.

By physical simulations, we also study the role of prestress. We show that in contrast to $1 \mathrm{D}$ and $2 \mathrm{D}$ networks, 3D networks experience an initial tension due to non-intersecting filaments resulting in initially stretched crosslinks, and are therefore prestressed. The modulus in the linear deformation regime is then governed by this prestress; indeed, it is higher than the modulus expected from the affine theory. Our simulations additionally indicate that if the network is allowed to relax initial tension by unbinding and rebinding of crosslinks, the impact of prestress on the elastic modulus in the linear regime becomes insignificant, although the prestress does not relax all the way to zero.

The remainder of the article is organized as follows. First, we present the affine theory of composite networks in Section 2.2.2. Under the assumption that deformations of the network are affine on the length scale of the filaments, we derive expressions for the stress and modulus in 1D, 2D, and 3D. We then present our physical simulation model and describe our network generation procedure in Section 2.2.3. We expand on the implications of our 3D simulation procedure in Section 2.2.4 in particular, we explain the emergence of prestress. We then discuss the results of our simulations in the linear deformation regime in Section 2.2.5 and indicate which results from the affine theory are still valid. Finally, we analyze the simulation results in the nonlinear regime in Section 2.2.6.

\subsubsection{Theory}

In this section we analytically derive the stress and modulus of a composite network under the assumption of affine deformations on the length scale of the filaments. We consider a collection of $N$ rigid filaments of length $L$ that are permanently connected by $n N / 2$ flexible crosslinks of contour length $l_{0}$, where $n$ is referred to as the crosslink density, i.e., the number of crosslinks per filament. The filaments are assumed to be 
perfectly rigid, i.e., they neither bend nor stretch, and the crosslinks are modeled via the WLC interpolation formula 23

$$
f_{\mathrm{cl}}(u)=\frac{k_{\mathrm{B}} T}{l_{\mathrm{p}}}\left(\frac{1}{4\left(1-\frac{u}{l_{0}}\right)^{2}}-\frac{1}{4}+\frac{u}{l_{0}}\right),
$$

where $k_{\mathrm{B}} T$ is the thermal energy, $l_{\mathrm{p}}$ the persistence length and $u \geq 0$ the end-to-end distance of the crosslink. Assuming $l_{0} \gg l_{\mathrm{p}}$ this force-extension relation implements a crosslink rest-length of zero and shows a characteristic stiffening with divergence of force as $u \rightarrow l_{0}$. Equation (2.1) can be integrated to yield the energy ${ }^{2}$ (up to a constant)

$$
E_{\mathrm{cl}}(u)=\frac{k_{\mathrm{B}} T}{l_{\mathrm{p}}}\left(\frac{l_{0}}{4\left(1-\frac{u}{l_{0}}\right)}-\frac{l_{0}}{4}-\frac{u}{4}+\frac{u^{2}}{2 l_{0}}\right) .
$$

Imposing affine deformations on the filament level fully determines the deformation field $u$ on the subfilament level. In the following analysis, we consider a single representative filament subject to an extensional strain of the surrounding medium that it is embedded in and crosslinked to.

\section{D network calculation}

We start with a one-dimensional system, i.e., 1D extensional strain $\epsilon$, and advance in dimensionality by converting an applied shear strain $\gamma$ to the orientation dependent extensional strain $\epsilon(\gamma)$ felt by the filament.

In the rest frame of the filament, the end-to-end distance of a crosslink at distance $x$ from the center of the filament is given by $|u(x, \epsilon)|=|\epsilon x|$ (see Fig. 2.5 (a)). For notational convenience, we consider positive $\epsilon$ only. Under the assumption that the crosslink density is high enough that one can consider the associated distribution as uniformly continuous, the total energy of a filament in 1D is given by

$$
E_{1 \mathrm{D}}(\epsilon)=2 \frac{n}{L} \int_{0}^{L / 2} E_{\mathrm{cl}}(\epsilon x) d x
$$

Substituting Eq. (2.2) into Eq. (2.3), this expression can be integrated analytically (see Section 2.2.9).

\footnotetext{
${ }^{2}$ More precisely, it is a free energy, which includes both, energetic (bending) and entropic terms for the crosslinks (not for the filaments).
} 
(a)
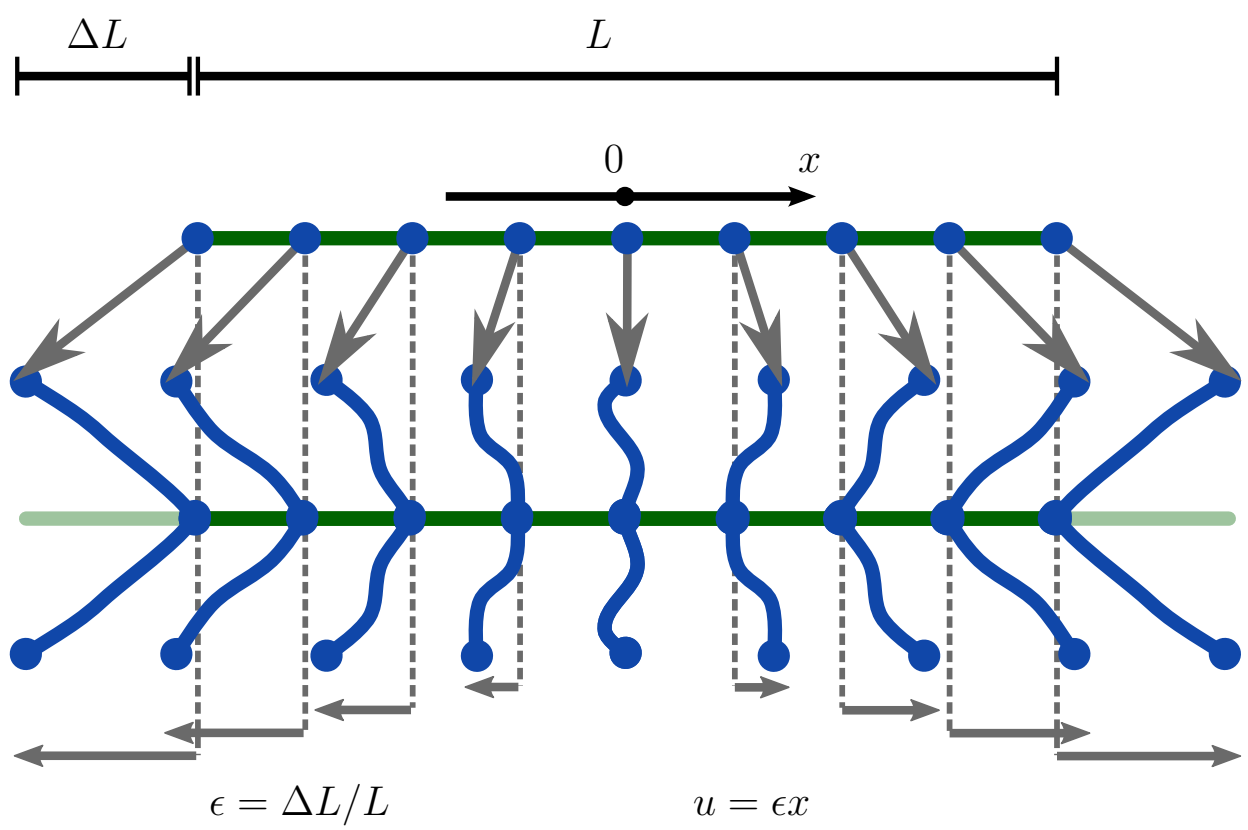

(b)
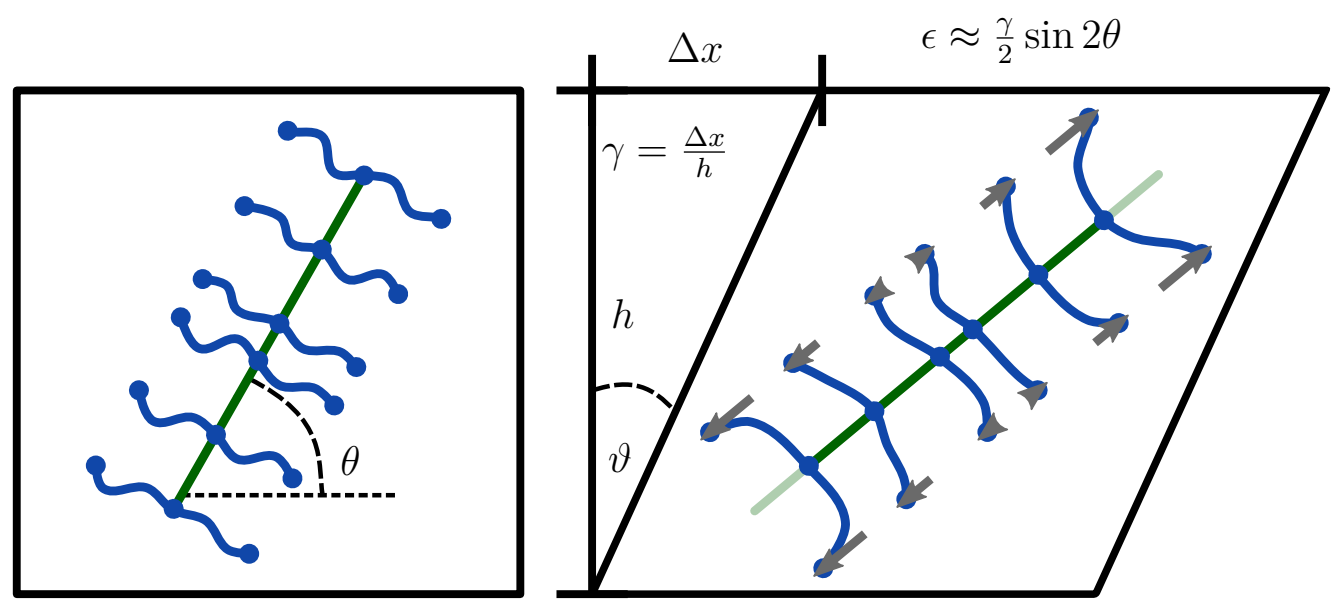

Figure 2.5.: Sketch of the assumptions of the affine theory: (a) 1D: A filament (green) of length $L$ is connected to its surrounding through $n$ crosslinks (blue) that have zero extension at zero strain. The surrounding of the filament is subject to a uniform extensional strain $\epsilon=\Delta L / L$. Since the filament itself is assumed to be perfectly rigid, all deformation goes into the crosslinks (drawn in y-direction for better visualization). The deformation of a crosslink at distance $x$ from the center of the filament is given by $u=\epsilon x$ (deformation field depicted by the horizontal gray arrows). (b) For a $2 \mathrm{D}$ system, the extensional strain on a filament at angle $\theta$ with the axis in shear direction is given by $\epsilon \approx(\gamma / 2) \sin 2 \theta$, for a small shear strain $\gamma=\Delta x / h=\tan \vartheta$. 
Following the described approach for the linear regime of the WLC force-extension relation, i.e., for $u \ll l_{0}$, the linear modulus may be extracted as $G_{0}^{\text {aff }}=\frac{2 E}{V \epsilon^{2}}$, where $E / V$ is the energy per unit volume $V$ in the network and $\epsilon$ is a small strain 27. For a $1 \mathrm{D}$ system this yields $G_{0}^{\text {aff }}=\frac{1}{8} \rho n k_{\mathrm{cl}} L$, with $k_{\mathrm{cl}}=\frac{3}{2} \frac{k_{\mathrm{B}} T}{l_{\mathrm{p}} l_{0}}$ being the linear spring constant of a crosslink and $\rho:=N L / V$ the total length of filaments per unit volume. The same holds for the modulus in $2 \mathrm{D}$ and $3 \mathrm{D}$, but with different numerical prefactors: $1 / 96$ and $1 / 192$, respectively $[16,19,26]$.

Next we show that one can extract a functional relation between nonlinear modulus and stress in the nonlinear regime, based on simple asymptotic scaling analysis. It follows from above that there is a strain $\epsilon_{\mathrm{d}}:=l_{0} /(L / 2)$ at which the outer most crosslink (at $x=L / 2$ ) reaches maximum extension. For $\epsilon \rightarrow \epsilon_{\mathrm{d}}$ the energy diverges as

$$
E_{1 \mathrm{D}}^{\mathrm{div}}(\epsilon) \sim-\frac{1}{\epsilon} \ln \left(1-\frac{\epsilon}{\epsilon_{\mathrm{d}}}\right)
$$

with ' $\sim$ ' defined via $E \sim f \Leftrightarrow E / f \rightarrow$ const. The upper index 'div' always indicates that we are only taking into account the diverging part of the $1 \mathrm{D}$ filament energy. We express stress and differential elastic modulus via $\sigma=\frac{1}{V} \frac{d E}{d \epsilon}$ and $K=\frac{1}{V} \frac{d^{2} E}{d \epsilon^{2}}$, respectively, in order to obtain $\sigma_{1 \mathrm{D}} \sim 1 /\left(1-\epsilon / \epsilon_{\mathrm{d}}\right)$, and $K_{1 \mathrm{D}} \sim 1 /\left(1-\epsilon / \epsilon_{\mathrm{d}}\right)^{2}$. We arrive at the asymptotic scaling relation

$$
K_{1 \mathrm{D}} \sim\left(\sigma_{1 \mathrm{D}}\right)^{2}
$$

This scaling relation between modulus and stress in 1D has also been derived in previous work [19]. Next we consider scaling relations in 2D and 3D.

\section{D network calculation}

We perform similar calculations as in $1 \mathrm{D}$, while taking into account that the extensional strain $\epsilon$, which results from a shear strain $\gamma$ on a $2 \mathrm{D}$ system, depends on the orientation of the filament under consideration. In the small-strain limit one thus obtains

$$
|\epsilon(\gamma, \theta)|=|(\gamma / 2) \sin 2 \theta|
$$

where $\theta \in[0, \pi]$ is the angle between the filament and the shear direction (see Fig. $2.5(\mathrm{~b})$ ). 
Substituting this expression into Eq. (2.4) and averaging over all orientations leads to

$$
\left\langle E_{2 \mathrm{D}}^{\mathrm{div}}\right\rangle_{\theta}(\gamma) \sim \int_{0}^{\pi / 2} \frac{-\ln \left(1-\frac{\gamma L}{4 l_{0}} \sin 2 \theta\right)}{(\gamma / 2) \sin 2 \theta} d \theta
$$

where we assume $\gamma \geq 0$ for notational convenience; the upper integration limit is reduced to $\pi / 2$ because $|\sin 2 \theta|$ is $\pi / 2$-periodic. Note that we do not take into account a redistribution of filament orientations under the shear transformation. This approach, as well as the small-strain approximation for $\epsilon(\gamma, \theta)$, are justified if $L \gg l_{0}$, since then the strain $\gamma_{\mathrm{d}}:=4 l_{0} / L$ at which the integrand diverges is small.

Differentiating Eq. (2.7) with respect to $\gamma$ and neglecting the weaker (logarithmically) diverging part of the integrand leads to an expression for the stress, as $\gamma \rightarrow \gamma_{\mathrm{d}}$ :

$$
\begin{aligned}
\left\langle\sigma_{2 \mathrm{D}}\right\rangle_{\theta}(\gamma) & \sim \int_{0}^{\pi / 2} \frac{d \theta}{1-\left(\frac{\gamma}{\gamma_{\mathrm{d}}}\right) \sin 2 \theta}, \\
& =\frac{\pi-\arccos \left(1-\gamma / \gamma_{\mathrm{d}}\right)}{\sqrt{1-\left(\gamma / \gamma_{\mathrm{d}}\right)^{2}}} .
\end{aligned}
$$

The divergence of the stress is of the form $\sigma_{2 \mathrm{D}} \sim 1 /\left(1-\left(\gamma / \gamma_{\mathrm{d}}\right)\right)^{1 / 2}$ and hence $K_{2 \mathrm{D}} \sim 1 /\left(1-\gamma / \gamma_{\mathrm{d}}\right)^{3 / 2}$. Therefore, the asymptotic scaling behavior for the differential modulus in two dimensions is given by

$$
K_{2 \mathrm{D}} \sim\left(\sigma_{2 \mathrm{D}}\right)^{3}
$$

Note the difference of the scaling relations to the ones in the 1D scenario. Stress shows a weaker divergence with strain than in 1D but a stronger dependence on the differential modulus. Integration of the diverging part of the stress further shows that the energy at maximum strain is finite - in contrast to the 1D setting, where the energy diverges at the critical strain. This is an effect introduced by orientational averaging only. 


\section{D network calculation}

For a 3D network, the extensional strain on a filament in the small-strain limit is given by

$$
|\epsilon(\gamma, \theta, \phi)|=|(\gamma / 2) \sin 2 \theta \cos \phi|
$$

in the usual spherical coordinates. In direct analogy to the 2D case (see Eq. (2.9p), the averaged stress close to $\gamma_{\mathrm{d}}=4 l_{0} / L$ can be written as

$$
\left\langle\sigma_{3 \mathrm{D}}\right\rangle_{\theta, \phi}(\gamma) \sim \int_{0}^{\pi / 2} \int_{0}^{\pi / 2} \frac{\sin \theta d \phi d \theta}{1-\left(\frac{\gamma}{\gamma_{\mathrm{d}}}\right) \sin 2 \theta \cos \phi},
$$

with $\gamma \geq 0$; the upper integration limit for the $\phi$ integration is reduced to $\pi / 2$ because $|\cos \phi|$ is $\pi$-periodic and symmetric about $\pi / 2$ on $[0, \pi]$. If we carry out the $\phi$ integral and expand the integrand around $\theta=\pi / 4$, in order to integrate over $\theta$ (see Section 2.2 .9 for details), we obtain $\sigma_{3 \mathrm{D}} \sim-\ln \left(1-\gamma / \gamma_{\mathrm{d}}\right)$ and hence $K \sim 1 /\left(1-\gamma / \gamma_{\mathrm{d}}\right)$. Consequently, $K$ does not scale with $\sigma$ as a power law; instead, one obtains

$$
K_{3 \mathrm{D}} \sim e^{c \sigma_{3 \mathrm{D}}}
$$

with a real constant $c$. The absence of asymptotic power law scaling sets $3 \mathrm{D}$ networks apart from $1 \mathrm{D}$ and 2D networks. In 3D, we observe the weakest (logarithmic) divergence of stress with strain. Integrating the diverging part of the stress shows that the energy again remains finite for $\gamma \rightarrow \gamma_{\mathrm{d}}$.

Finite crosslink density. By considering the limit of infinite crosslink density, we have derived theoretical scaling relations for strain stiffening by integrating along a filament's backbone (see Eq. (2.3)). For any real system, however, the crosslink density is finite and Eq. (2.3) turns into a sum

$$
E=\sum_{i=1}^{n} E_{\mathrm{cl}}\left(\epsilon x_{i}\right)
$$

where $\left\{x_{i}\right\}$ are the crosslink binding sites along the filament. Fig. 2.6 shows numerical results for the behavior of the corresponding differential modulus $K$ for finite $n$, obtained by numerical evaluation of Eq. (2.14) and proper orientational averaging. 
Note that the asymptotic scaling behavior of $K$ in the limit of infinite crosslink density influences a finite network's behavior in the intermediate-stress regime (see inset of Fig. 2.6); however, near the critical strain, the differential modulus scales as $K \sim \sigma^{3 / 2}$, i.e., like the response of a single WLC. Furthermore, for 1D and 2D systems the theoretical scaling exponents in the limit of infinite crosslink densities can (in the intermediate regime) indeed be approached by increasing $n$. In contrast, as shown above, in 3D the theoretically derived scaling of $K$ is exponential in $\sigma$. Such an exponential increase is quantified by an (in principle) indefinitely increasing maximal slope with increasing $n$ in the $\ln K$ versus $\ln \sigma$ plots; e.g., for $n=60$ the maximal slope is 3.49 , for $n=3000$ it is 5.82. However, for any finite $n$, eventually there is always a universal scaling of $K \sim \sigma^{3 / 2}$, resulting from the single WLC force-extension relation, independent of the dimensionality of the system. Indeed, for any given $n$, the integral representation Eq. 2.3 becomes invalid close to $\gamma=\gamma_{\mathrm{d}}$ due to the divergence of the WLC energy.

The numerical results in Fig. 2.6 have been obtained without the small-strain approximation for the extension of the filaments. However, redistribution of the filament orientations under shear has not been taken into account in Fig. 2.6. Calculations including this effect show that it may both decrease and increase the maximum intermediate slope in the $\ln K$ versus $\ln \sigma$ plot and shift the peak to larger stress values depending on the maximum strain $\gamma_{\mathrm{d}}$. In any case, the asymptotic scaling regime remains unchanged.

In the next section we introduce the simulation framework that we use to study 3D networks consisting of many filaments and crosslinks, relaxing the assumption of affine deformations.

\subsubsection{Simulation model}

We perform quasistatic simulations of 3D networks that consist of $N$ rigid filaments of length $L$, permanently crosslinked by a collection of $n N / 2$ crosslinks of length $l_{0}$. All lengths are measured in units of the side length of the cubic periodic simulation box. A typical set of parameters is $N=3000, L=0.3, n=60, l_{0}=0.03$.

Each filament is modeled as perfectly rigid, implying that its configuration can be described by its two endpoints only, which are constraint to stay at distance $L$. The flexible crosslinks are modeled as a central force acting between the two binding sites. In particular, we use the WLC interpolation formula (Eq. (2.1) ) and the corresponding energy (Eq. (2.2)). In all data that is presented, forces are measured in 


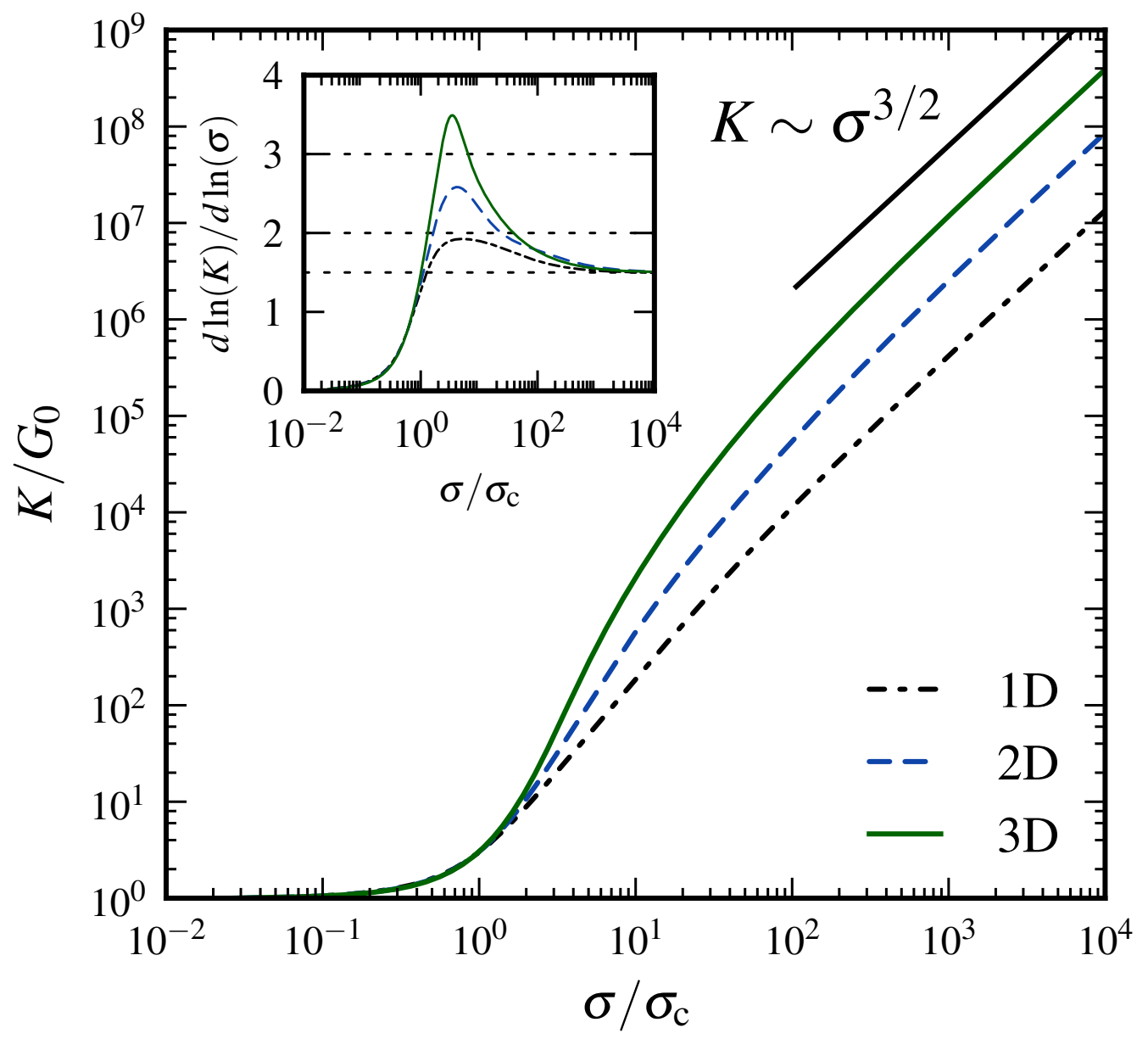

Figure 2.6.: Differential modulus $K$ as a function of shear stress $\sigma$ in the affine limit, with finite number of crosslinks $(n=60)$, rescaled by the linear elastic modulus $G_{0}:=\left.K\right|_{\gamma=0}$ and critical stress $\sigma_{\mathrm{c}}:=\sigma\left(\gamma_{\mathrm{c}}\right)$, respectively, where $\gamma_{\mathrm{c}}$ is defined via $K\left(\gamma_{\mathrm{c}}\right)=3 G_{0}$. Straight line indicates power law scaling $K \sim \sigma^{3 / 2}$. Inset shows local slope $d \ln K / d \ln \sigma$; dotted lines indicate power law scaling with exponents from affine theory $\{2,3\}$ and single WLC scaling $\{3 / 2\}$. Independent of dimensionality, the asymptotic large stress scaling is $K \sim \sigma^{3 / 2}$. In an intermediate-stress regime, the theoretical values for infinite crosslink densities are approached. 
units of $\left(k_{\mathrm{B}} T\right) / l_{\mathrm{p}}$. There are no additional degrees of freedom introduced through the crosslinks, since their configuration is represented via the endpoints of the filaments, in terms of barycentric coordinates.

In order to generate an initial network configuration we proceed as follows. We generate $N$ randomly distributed filaments by first randomly choosing their centers of mass in our simulation box and by then picking a random orientation for each filament. For crosslinking we apply the following iterative procedure. We randomly choose two points on distinct filaments and insert a crosslink if the corresponding point-to-point distance is shorter than a certain threshold $\alpha l_{0}$. Here $\alpha \in[0,1)$ serves as a parameter to vary the initially allowed crosslink lengths in the system. This procedure is repeated until the desired number of crosslinks is reached; see Fig. 2.7 for an illustration of the final configuration. Since we perform quasistatic simulations, the system must be at static equilibrium at all times. As practically all crosslinks will be stretched beyond their rest-length after the initial network generation, we minimize the energy (of the crosslinks) before subjecting the simulation box to any deformation ${ }^{3}$. For energy minimization we use the freely available external library IPOPT [28], which requires the gradient and the Hessian of the system's energy function. It might happen during the optimization process, that individual crosslinks reach extensions $u$ larger than their contour length $l_{0}$. Acceptance of these solutions is prohibited by setting the energy to infinity $\left(10^{19}\right)$ for $u \geq l_{0}$ in Eq. 2.2 ; without this modification it would become negative in that regime. The length constraints for the filaments are realized via Lagrange multipliers.

In order to extract elastic properties of the network we perform quasistatic shearing by applying an affine incremental shear strain $\delta \gamma$ to the network, with subsequent rescaling of filaments to length $L$ (see Fig. 2.5). We apply Lees-Edwards shearing periodic boundary conditions 29]. The magnitude of $\delta \gamma$ is determined by calculating the maximum affine shear that leaves all crosslinks below their contour length. Due to the rescaling of filament lengths, a nonaffine deformation component is introduced. This nonaffinity may lead to crosslinks being overstretched after all. In this case, we iteratively halve the shear strain until the length of all crosslinks remains below their contour length. After each shear increment, the energy is minimized. We apply a fixed upper bound of $1 \%$ strain on $\delta \gamma$ in order to stay reasonably close to the previous solution. This increases numerical efficiency and accelerates convergence because it allows us to use a warm-start procedure that reuses Lagrange multipliers

\footnotetext{
${ }^{3}$ We do neither take into account fluctuations of the filaments nor excluded-volume effects.
} 


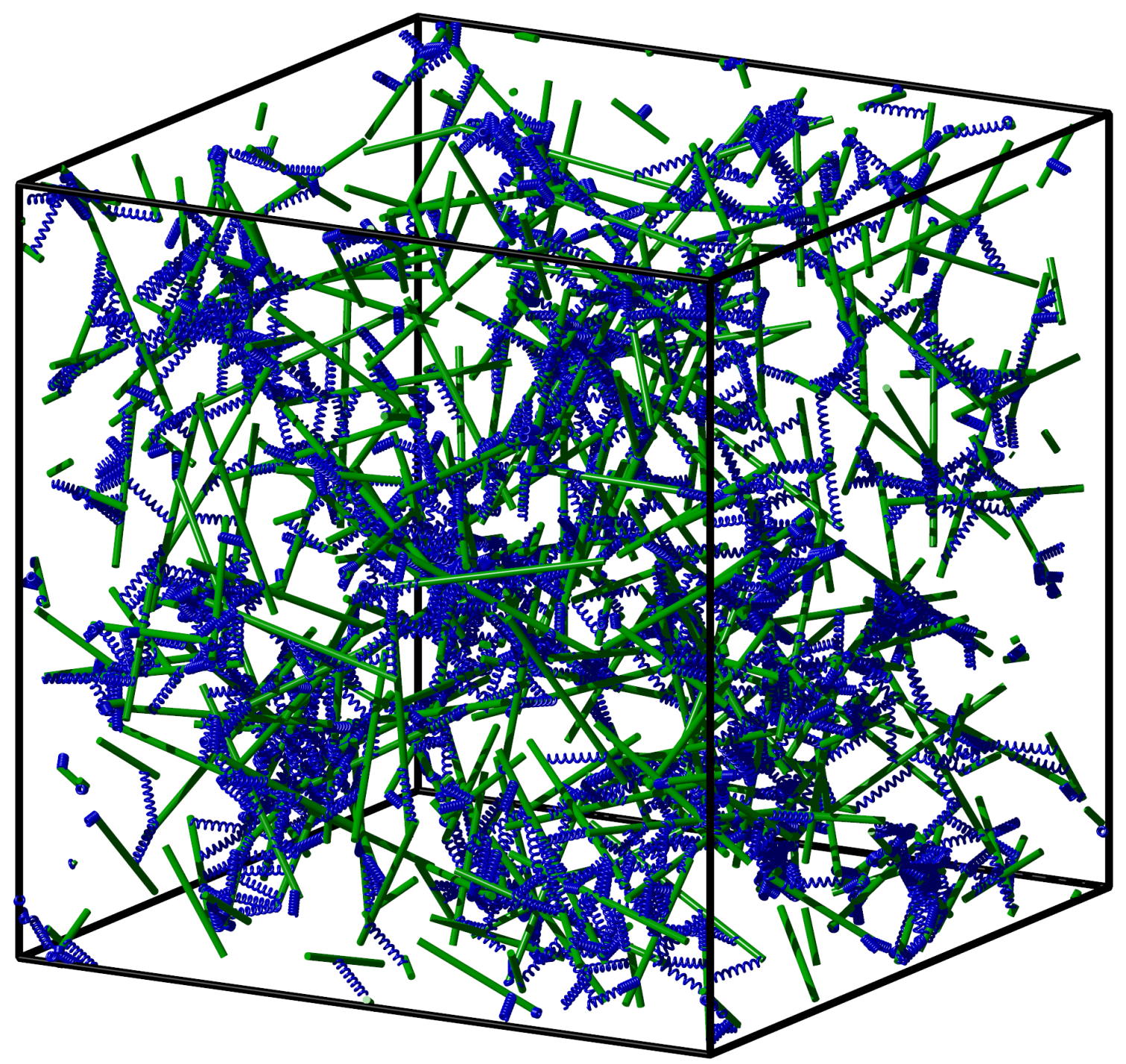

Figure 2.7.: Example of an initially generated network that has not been relaxed into static equilibrium yet. Rigid filaments are shown in green, flexible crosslinks in blue. Short crosslink or filament fragments correspond to filaments/crosslinks that cross the periodic boundaries of the simulation box. For the sake of visual appearance, the network is much sparser than the systems that are studied in the remainder of this article, and the ratio of filament to crosslink length is much smaller, $N=300, n=10, L=0.3, l_{0}=0.1, \alpha=0.9$. 
from one minimization as initial guesses for the next one. Moreover, the application of small shear steps reduces the likelihood of discontinuously jumping between local energy minima.

We stop shearing when the achievable increment in shear strain becomes smaller than a chosen threshold due to crosslinks that are very close to their maximum extension. During the entire simulation process, we record network parameters in the equilibrated states - in particular, the energy $E$ as a function of shear strain $\gamma$. This allows us to extract the shear stress $\sigma=\frac{1}{V} \frac{d E}{d \gamma}$ as well as the differential shear elastic modulus $K=\frac{d \sigma}{d \gamma}=\frac{1}{V} \frac{d^{2} E}{d \gamma}$. Derivatives are taken by first interpolating $E(\gamma)$ with a cubic spline. We define the linear shear elastic modulus as

$$
G_{0}:=\left.K\right|_{\gamma=0}
$$

In the following section we discuss the implications of our specific simulation model, in particular with respect to network structure, and contrast it with previous studies that have been carried out mostly in 2D.

\subsubsection{Initial tension and prestress}

As mentioned in Section 2.2.3, our network generation results in a non-zero initial energy $E_{0}$ at zero strain. Indeed, by randomly placing (zero-diameter) filaments in a 3D container, filaments have zero probability to intersect; thus, crosslinks have finite initial extension with probability one. This is different from $2 \mathrm{D}$, where randomly placed filaments mutually intersect with a probability approaching one as their number increases. Indeed, so-called Mikado models $[19,30,32$, where filaments are crosslinked at their intersection sites only, exhibit no forces at zero strain.

In contrast, the initial stretching of crosslinks in our networks results in an initial tension before any deformation. For a quantitative analysis we measure a global variant of this effect by what we call total prestress $\sigma_{0}$, which measures the normal stress $s^{4}$ component orthogonal to the shear planes 5 . More precisely, we measure the single sided (e.g., upward) normal component of the force that is acting on a given shear plane, by summing up the normal components of the forces exerted by each

\footnotetext{
${ }^{4}$ Note that our notion of prestress is not to be confused with the constant prestress externally applied in bulk rheology experiments, which is a shear stress in general.

${ }^{5}$ Although we could in principle define total prestress as the normal component of the stress acting on any plane in our system we prefer to use shear planes as this simplifies the forthcoming analysis.
} 
(a)

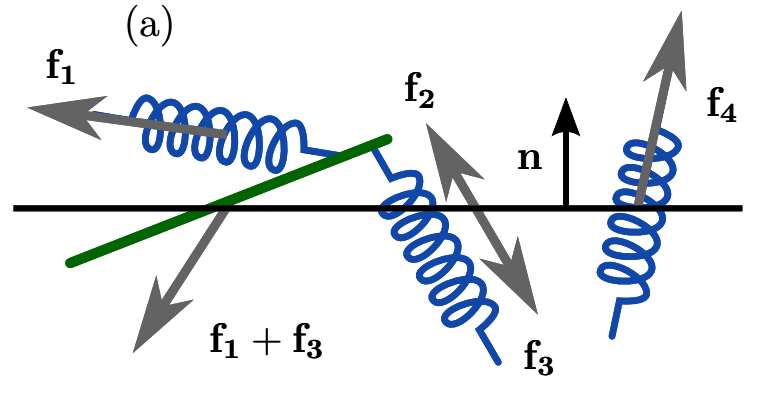

(b)

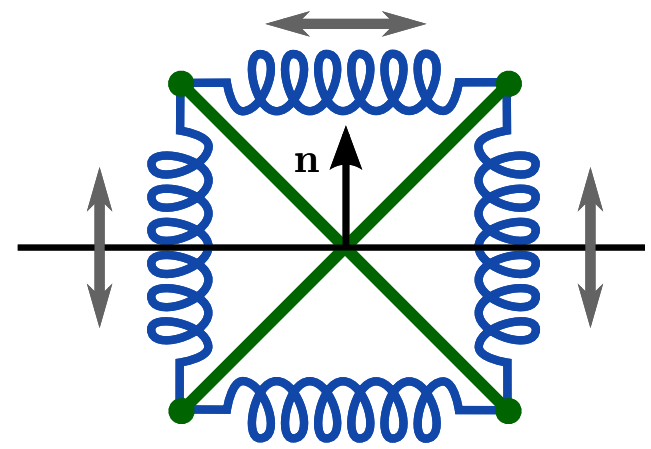

Figure 2.8.: (a) Measuring the total prestress $\sigma_{0}$ by extracting the normal component of the total force acting on a shear plane. We sum up all the forces acting on one side of the plane exerted by (i) the crosslinks passing through (here $\mathbf{f}_{\mathbf{2}}$ and $\mathbf{f}_{\mathbf{4}}$ ) and (ii) the filaments passing through (here $\mathbf{f}_{\mathbf{1}}+\mathbf{f}_{\mathbf{3}}$ ) - then we project onto the normal vector n. (b) A tensegrity structure (here: Snelson's $X$ [33]) remains in static equilibrium without application of boundary conditions. The forces acting on any plane add up to zero, i.e., no plane carries any total prestress although it is under tension locally.

crosslink and filament passing through the given shear plane, see Fig. 2.8 (a). The normal stress is then given by dividing by the surface area of the shear plane. Note that $\sigma_{0}$ does not depend on the choice of a particular shear plane; indeed, if the total stress was changing during vertical movement of a shear plane, then this would immediately contradict force balance in the system.

Intuitively, one might expect negative normal stresses (pulling down on the upper face of the simulation box), since crosslinks are contractile. However, since filaments withstand compression, it is possible to construct systems that exhibit positive normal stress. This suggests the existence of configurations with zero normal stres $\$^{6}$ Indeed, so-called tensegrity structures [34], which are in static equilibrium in the absence of boundary conditions satisfy this criterion - while still being able to store arbitrary amounts of energy (see Fig. 2.8(b)). Empirically, our simulations show that the random networks generated by the procedure described in Section 2.2.3 exhibit negative initial normal stresses throughout. Their integrity is provided through the application of periodic boundary conditions. Note in particular, that our setup enforces conservation of volume of the simulation box. In general, it would be possible to relax the prestress by letting the volume of the simulation box change. However,

\footnotetext{
${ }^{6}$ Note that individual crosslinks are still under tension; however, the total normal force acting on the shear plane vanishes.
} 
we did not follow this approach in the study presented here, in order to ensure that the filament length remains significantly smaller than the size of the simulation box.

In the following, we relate total prestress to the linear elastic response of our networks.

\subsubsection{Linear regime}

In previous work 16, 19, 26, an expression for the linear modulus in 3D was derived under the assumption of affine deformations and in absence of any initial tension in the network. Our simulations show that the linear elastic modulus depends on the initial tension in the network.

One scenario that clearly demonstrates the dependence of the linear modulus $G_{0}$ (defined in Eq. 2.15) on the initial tension is illustrated in Fig. 2.9 where the admissible maximum initial crosslink length was varied.

For a more quantitative analysis we have designed a method that allows us to change initial tension for a network with a fixed set of simulation parameters. We first randomly generate a network as described above and let it relax into static equilibrium. We then remove a given amount (5\%) of the most-stretched crosslinks in the system. Then we reconnect those crosslinks randomly again, and let the network relax. This procedure is repeated $N_{\text {rel }}$ times. Thereby, we successively decrease the system's initial tension, and therefore also its total energy, see inset of Fig. 2.10, Not only does the total energy decrease, we also observe a change in the distribution of forces (see Fig. 2.10). As long as one performs the crosslink binding-unbinding procedure over a small enough fraction of crosslinks, the network remains nearly isotropic.

It is apparent from the inset of Fig. 2.11 that the linear elastic modulus is reduced by increasing the number of relaxation steps, as expected. Fig. 2.11 also shows the dependence of linear modulus $G_{0}$ on the total prestress $\sigma_{0}$, which has been introduced in Section 2.2.4. We varied $\sigma_{0}$ via the above described procedure, and measured $G_{0}$ with the shearing protocol described in Section 2.2.3. After a certain number of relaxation steps the empirical value for $G_{0}$ equals the value $G_{0}^{\text {aff }}$ expected from affine theory (see Section 2.2.2). Relaxing initial tension further, we reach moduli even below $G_{0}^{\text {aff }}$. This is possible because the network can rearrange nonaffinely, thereby softening its response. Over a certain range of total prestresses, we observe linear scaling of $G_{0}$ with $\sigma_{0}$, a phenomenon, which has been discussed in other contexts before (see for example Ref. [35]). We explain the linear regime as follows. For 


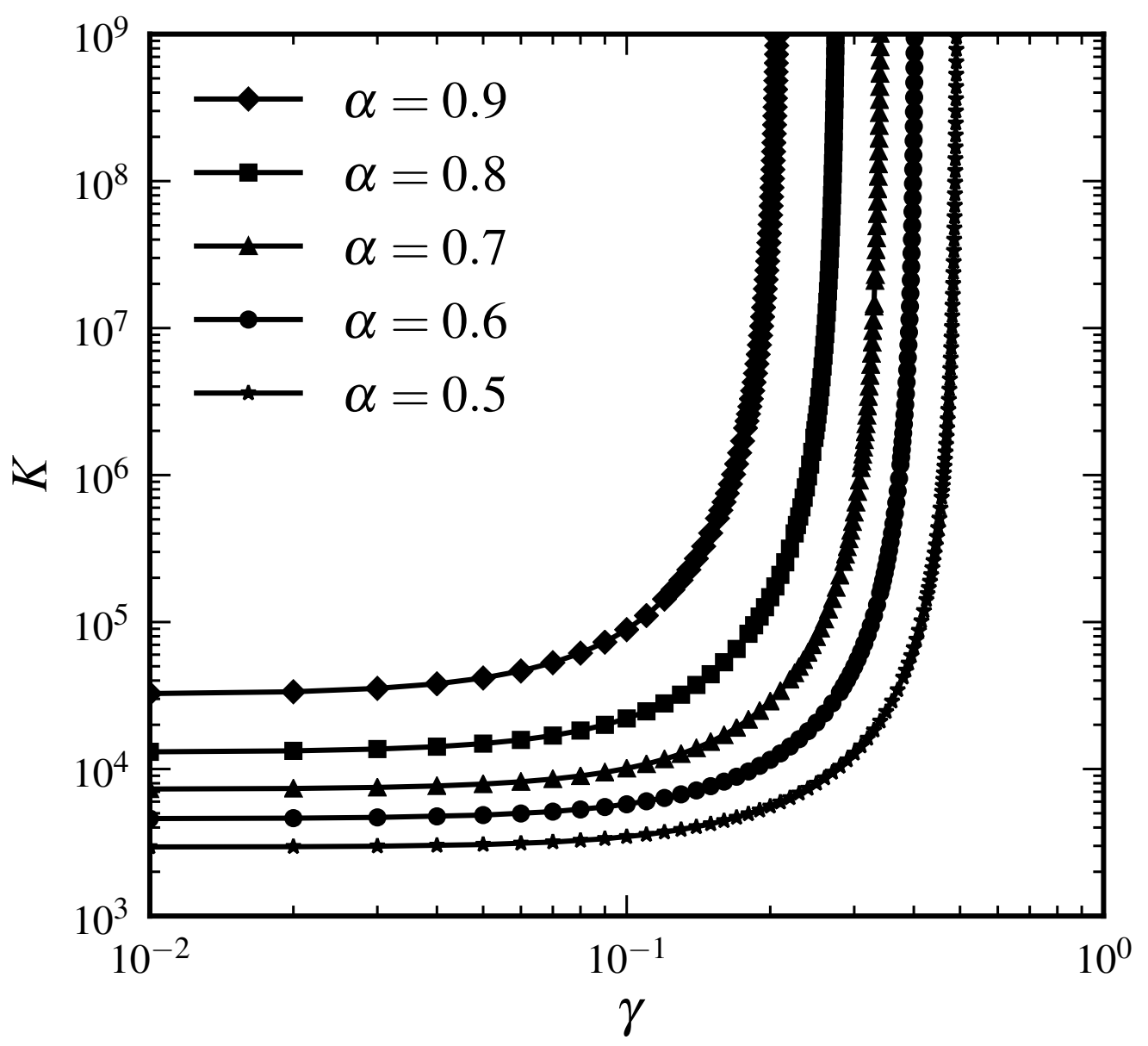

Figure 2.9.: Differential elastic modulus $K$ as a function of strain $\gamma$ for different levels of initial tension. The initial tension in the network is varied by changing the initially admissible maximal crosslink length $\alpha l_{0}$. The linear modulus $G_{0}=\left.K\right|_{\gamma=0}$ increases with the initial tension in the network (initial tension increases with $\alpha$ ). It is also evident that the divergence of $K$ occurs at a strain $\gamma_{\mathrm{d}}$ that decreases with increasing $\alpha$. Here: $N=3000, n=60, L=0.3, l_{0}=0.03$. 


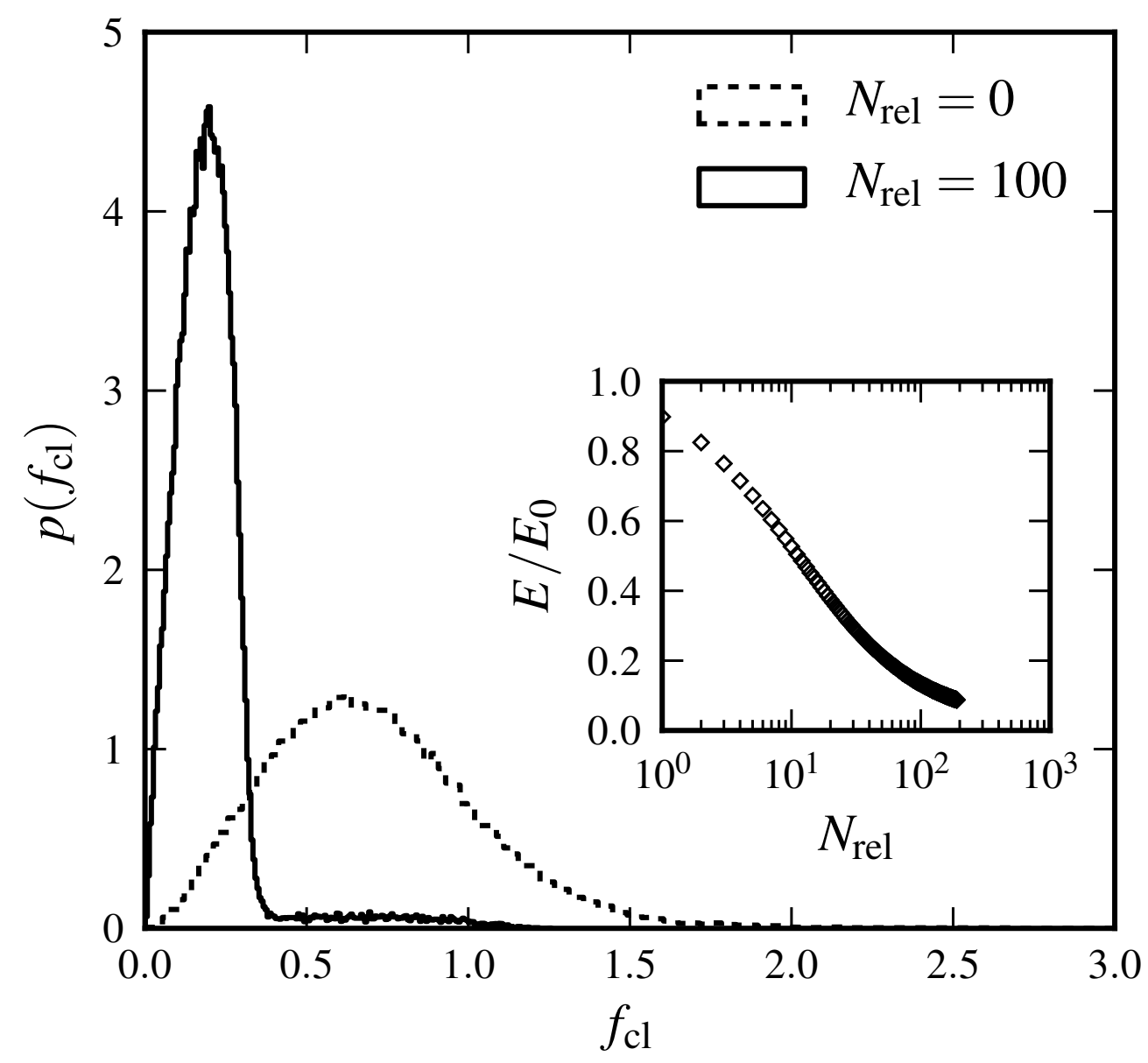

Figure 2.10.: Distribution of forces in crosslinks for a system without or with $N_{\text {rel }}=100$ relaxation steps. The relaxation procedure cuts the large force tail of the initial distribution and establishes a sharper peak at small forces. The inset shows the total energy $E$ in the system, normalized by the initial energy $E_{0}$, as a function of number of relaxation steps $N_{\text {rel }}$. 


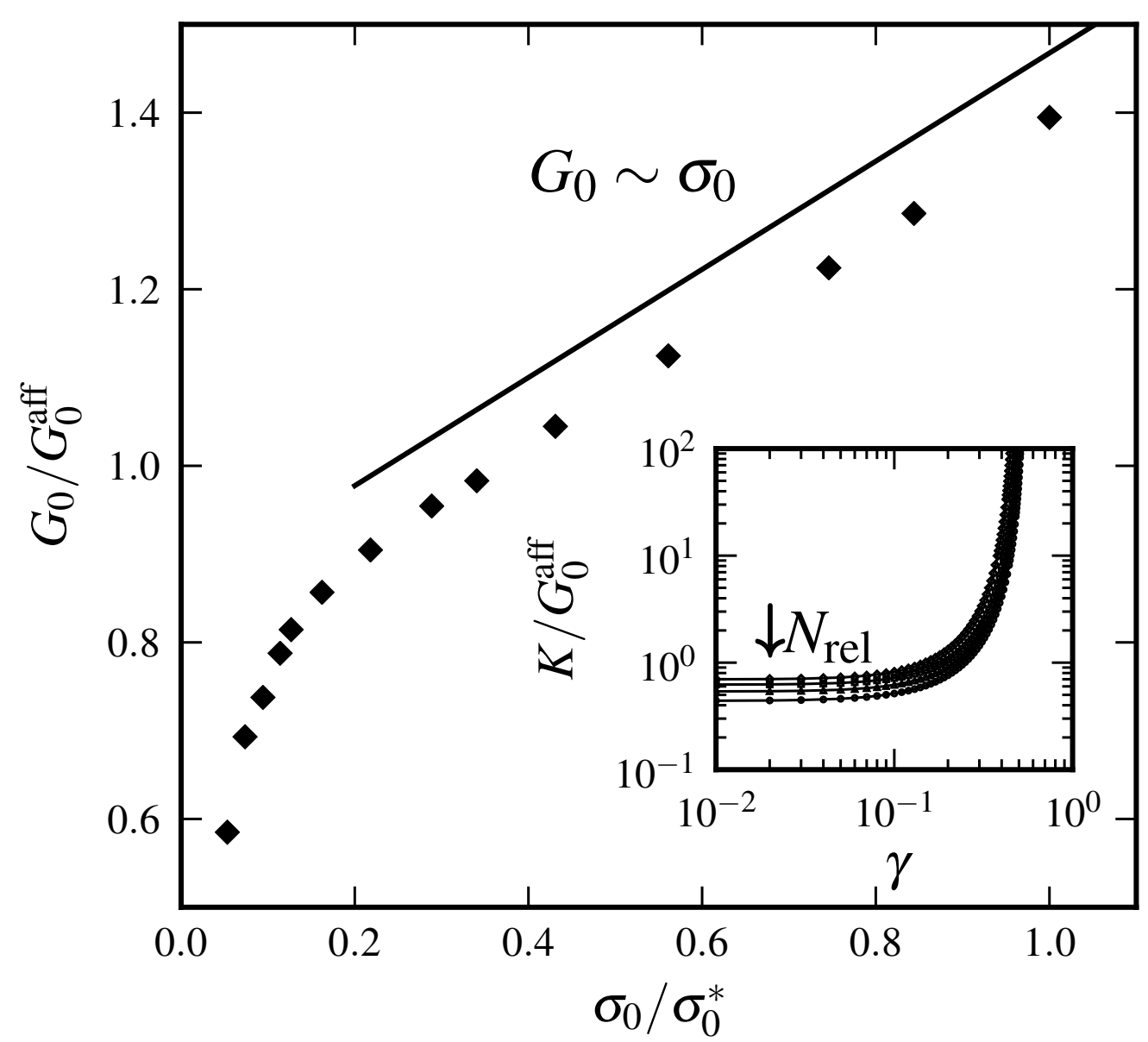

Figure 2.11.: Linear elastic modulus $G_{0}$ normalized by the affine prediction $G_{0}^{\text {aff }}$ as a function of total prestress $\sigma_{0}$ normalized by the total prestress $\sigma_{0}^{*}$ immediately after initial network generation. The total prestress is reduced via the procedure described in Section 2.2.5. For small total prestress, $G_{0}$ exhibits superlinear dependence on $\sigma_{0}$. Up to $\sigma_{0}=\sigma_{0}^{*}$, we observe linear scaling $G_{0} \propto \sigma_{0}$, as predicted by the model. The straight line is drawn as a guide to the eye, representing linear scaling. Parameters: $N=3000, n=60, L=0.3, l_{0}=0.06, \alpha=0.5$. The inset shows differential elastic modulus $K$ versus shear strain $\gamma$ for systems with varying number of relaxation steps $N_{\text {rel }} \in\{0,50,100,150\} . G_{0}$ goes down with increasing $N_{\text {rel }}$. Parameters: $N=3000$, $n=60, L=0.3, l_{0}=0.03, \alpha=0.5$. 

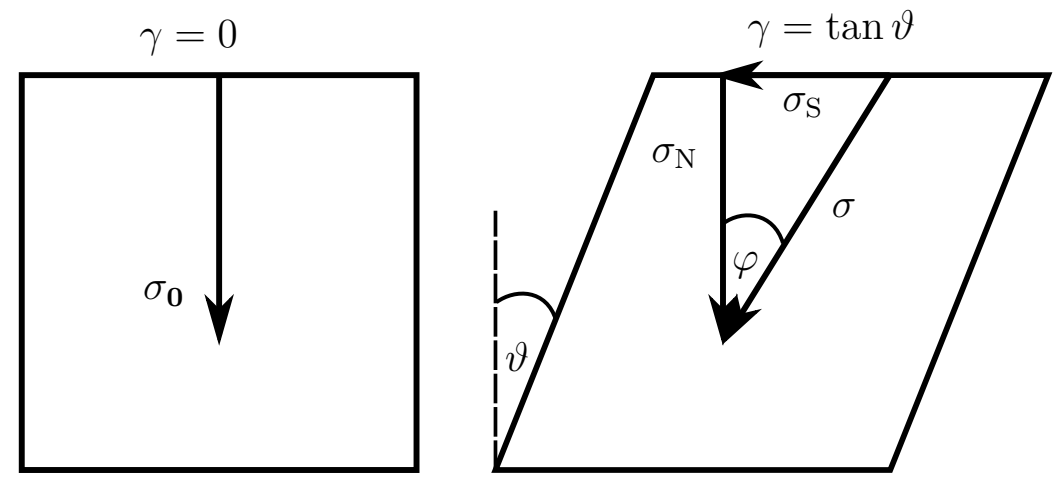

Figure 2.12.: The initial network carries a total prestress $\sigma_{0}$. After a small shear $\gamma=\tan \vartheta$ has been applied it exhibits a shear stress $\sigma_{\mathrm{S}}$ and normal stress $\sigma_{\mathrm{N}}$, with $\tan \varphi=\sigma_{\mathrm{S}} / \sigma_{\mathrm{N}}$.

small strains the normal component $\sigma_{\mathrm{N}}$ of the stress acting on shear planes is close in magnitude to the total prestress $\sigma_{0}$, i.e., $\sigma_{\mathrm{N}} \approx \sigma_{0}$. For small strains given by shear angles $\vartheta \approx 0$, total forces acting on the shear planes make an angle $\varphi$ with the direction normal to the shear planes (see Fig. 2.12). Our simulations show that $\tan \varphi \propto \tan \vartheta$ and that the constant of proportionality remains unchanged in the linear scaling regime. Therefore, shear satisfies

$$
\gamma=\tan \vartheta \propto \frac{\sigma_{\mathrm{S}}}{\sigma_{0}}
$$

where $\sigma_{\mathrm{S}}$ is the component of the stress acting on shear planes in the shear direction, see Fig. 2.12, Hence, the linear elastic shear modulus $G_{0}$ defined via $\sigma_{\mathrm{S}}=G_{0} \gamma$ is proportional to the total prestress $\sigma_{0}$ via Eq. (2.16). However, for very small total prestresses, i.e., after many relaxation steps, the modulus shows a steeper than linear dependence on $\sigma_{0}$. Indeed, in this regime the aforementioned constant of proportionality becomes larger. This effect might be attributed to the fact that for small $\sigma_{0}$, tensegrity type elements (see Fig. 2.8 (b)), which do not contribute to the total prestress but carry energy, contribute significantly to the measured shear stress, thereby increasing $\varphi$ (see Fig. 2.12).

Furthermore, affine theory predicts linear scaling of the modulus $G_{0}$ with crosslink density $n$. Fig. 2.13 shows that this linear scaling is indeed reproduced in our simulations, independent of the prestress. Moreover, by changing the prestress via our relaxation procedure it is possible to reach comparable slopes to what is predicted by the affine theory. 


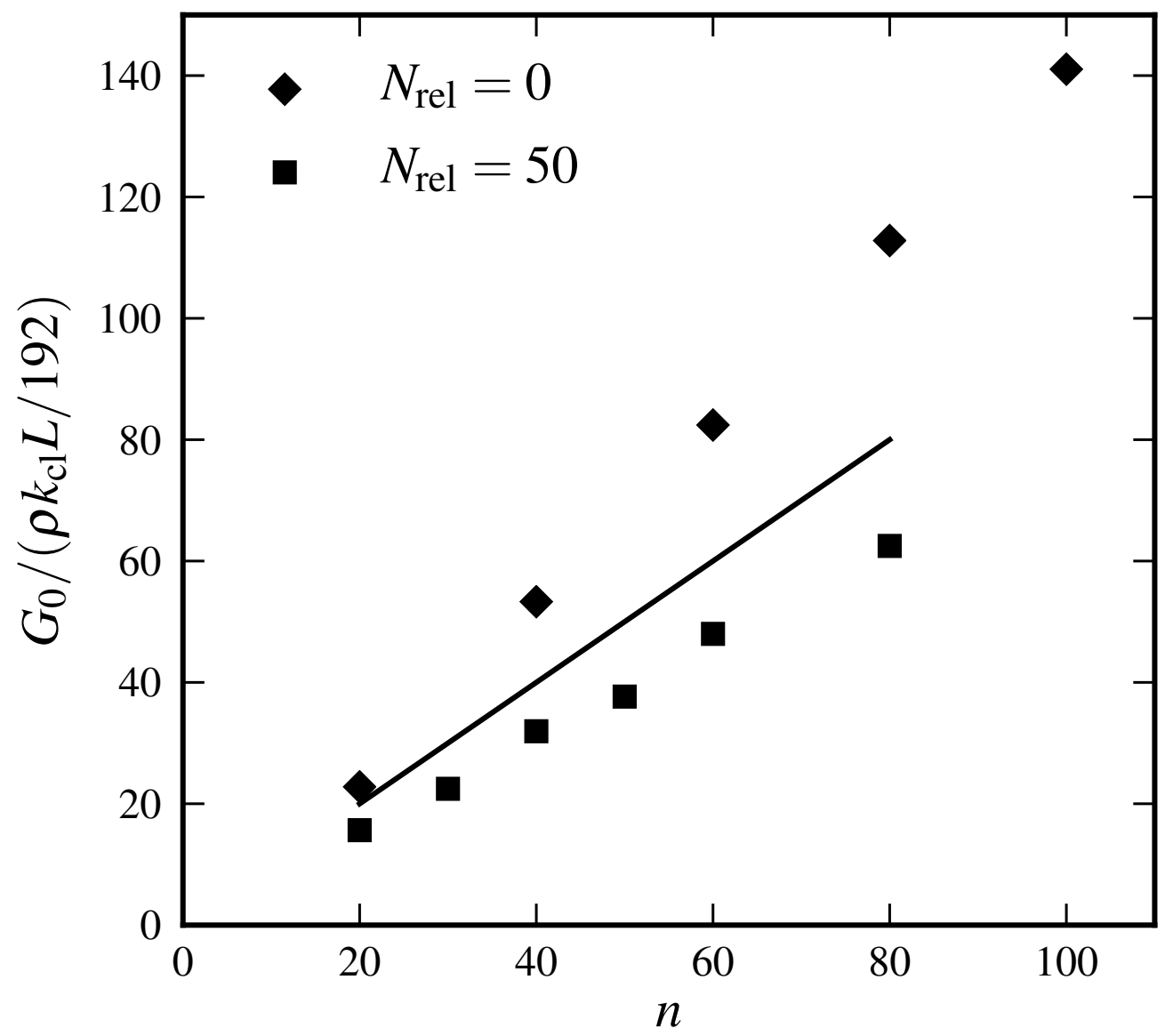

Figure 2.13.: Linear elastic modulus $G_{0}$ versus crosslink density $n$ for systems with different number of relaxation steps: $N_{\text {rel }}=0$ (diamonds) and $N_{\text {rel }}=50$ (squares). Solid line indicates values expected from affine theory: $G_{0}^{\text {aff }}=\rho n k_{\mathrm{cl}} L / 192$. Parameters: $N=3000, L=0.3, l_{0}=0.06, \alpha=0.5$. 
The next section deals with the nonlinear elastic response of the simulated networks, and relates it to the theoretical results that were derived in Section 2.2.2.

\subsubsection{Nonlinear regime}

\section{Critical strain}

The networks that we study are inherently nonlinear because crosslinks are WLCs with finite length $l_{0}$ (see Eq. (2.1)), resulting in pronounced strain stiffening at a critical strain $\gamma_{\mathrm{c}}$. Stress diverges at a higher strain $\gamma_{\mathrm{d}}$. In our simulations, we define the critical strain $\gamma_{\mathrm{c}}$ to be the strain where $K / G_{0} \approx 3$. In the affine theory, $\gamma_{\mathrm{d}}$ and $\gamma_{\mathrm{c}}$ scale linearly with the ratio of crosslink to filament length $l_{0} / L$. In our simulations, we cannot conclusively report on this dependence because the accessible ranges for $l_{0}$ and $L$ are quite limited. On the one hand, there exists an upper limit for $L$ (therefore also for $l_{0}$, since $l_{0} / L \ll 1$ should hold) to be significantly smaller than the simulation box. On the other hand, $L$ and $l_{0}$ are bounded from below due to computational limitations - this is because we need to increase the number of filaments in order to keep networks homogenous.

For ranges that are accessible to our simulations, we obtain the following results. If we fix $l_{0}$, then we observe linear scaling $\gamma_{\mathrm{c}} \propto 1 / L$ for systems where no relaxation procedure has been applied (see Fig. 2.14(a)). Relaxed systems, however, sometimes show a less than linear dependence. This effect might be due to anisotropies induced by the relaxation procedure. If we fix $L$, then the dependence of $\gamma_{\mathrm{c}}$ on $l_{0}$ is slightly less than linear (see Fig. 2.14 (b)).

\section{Differential modulus}

It remains to discuss the dependence of the differential modulus on stress, the affine theory of which has been derived in Section 2.2.2. For finite crosslink densities, the only persistent scaling behavior is $K \sim \sigma^{3 / 2}$, as $\gamma$ approaches $\gamma_{\mathrm{d}}$-due to the fact that eventually single WLC response dominates. In an intermediate regime, above the critical stress $\sigma_{\mathrm{c}}=\sigma\left(\gamma_{\mathrm{c}}\right)$, we observe slopes $(d \ln K / d \ln \sigma)>3 / 2$. The majority of the simulations shows intermediate slopes around 2 or slightly above, mostly independent of simulation parameters, but there are also realizations that show maximum slopes up to 3.5 (see Fig. 2.15). These higher slopes and the final scaling $K \sim \sigma^{3 / 2}$ are in accordance with the predictions of affine theory. Indeed, a slope of 3.5 is the maximum slope predicted by the affine theory when using the 


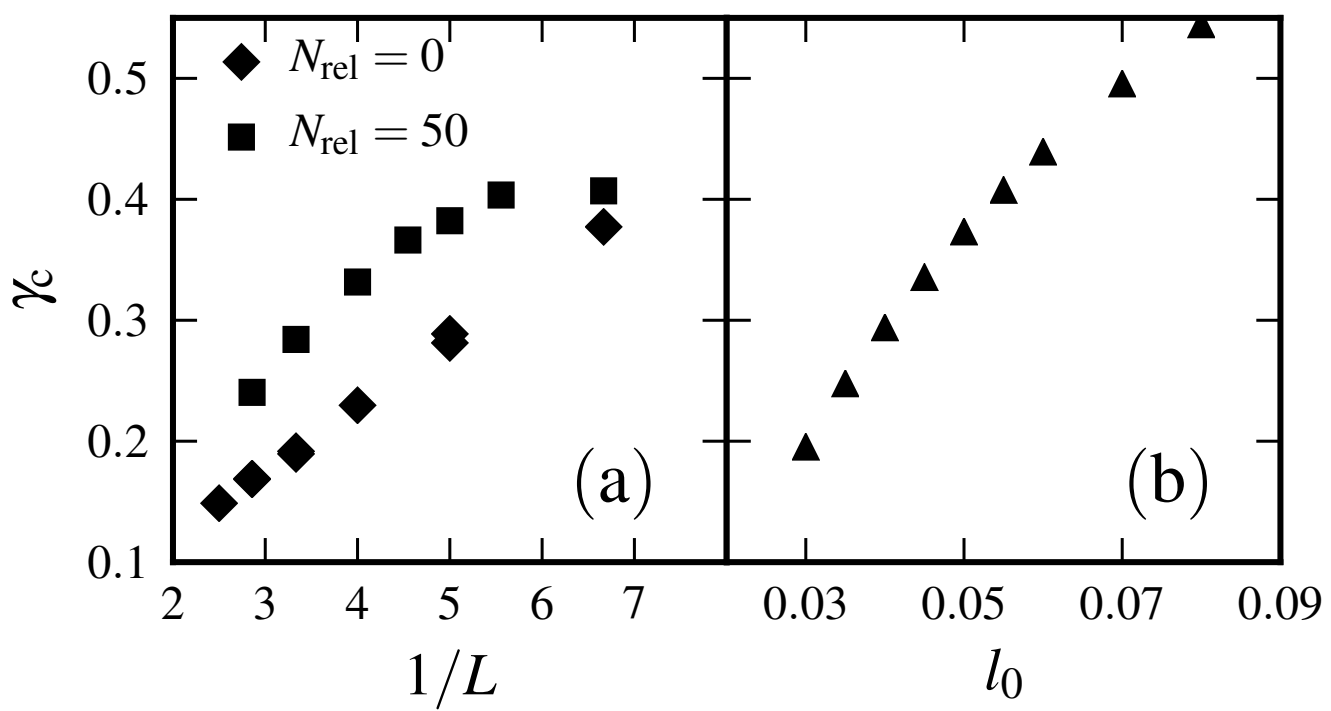

Figure 2.14.: (a) Critical strain $\gamma_{\mathrm{c}}$ versus inverse filament length $1 / L$ for $N_{\text {rel }}=0$ and $N_{\text {rel }}=50$. Other parameters: $N=5000, n=60, l_{0}=0.04, \alpha=0.7$. We observe linear scaling $\gamma_{\mathrm{c}} \propto 1 / L$ for $N_{\text {rel }}=0$; systems in which relaxation has been applied show deviations from this behavior (see $N_{\text {rel }}=50$ here). (b) Critical strain $\gamma_{\mathrm{c}}$ versus crosslink contour length $l_{0}$ for a system with $N=3000, n=50, L=0.3, \alpha=0.5$.

same crosslink density as in the simulation (Fig. 2.6). There are, however, differences between theory and simulation in terms of slope profiles since various assumption are made by the theory that do not hold in the simulations: A randomly generated network does not have a uniform crosslink density along the filaments, these systems are prestressed, and there is no perfect isotropy. Moreover, the networks do not deform perfectly affinely.

\section{Nonaffinity}

In order to study to what extent simulation results deviate from affine theory, apart from prestress, nonuniform crosslink density, and anisotropy, we analyze the nonaffinity of the network deformation under shear. For a single filament, we define its differential nonaffinity with respect to the center of mass by

$$
\frac{\left\|\delta r_{\mathrm{aff}}-\delta r\right\|^{2}}{\|\delta \gamma\|^{2}}
$$




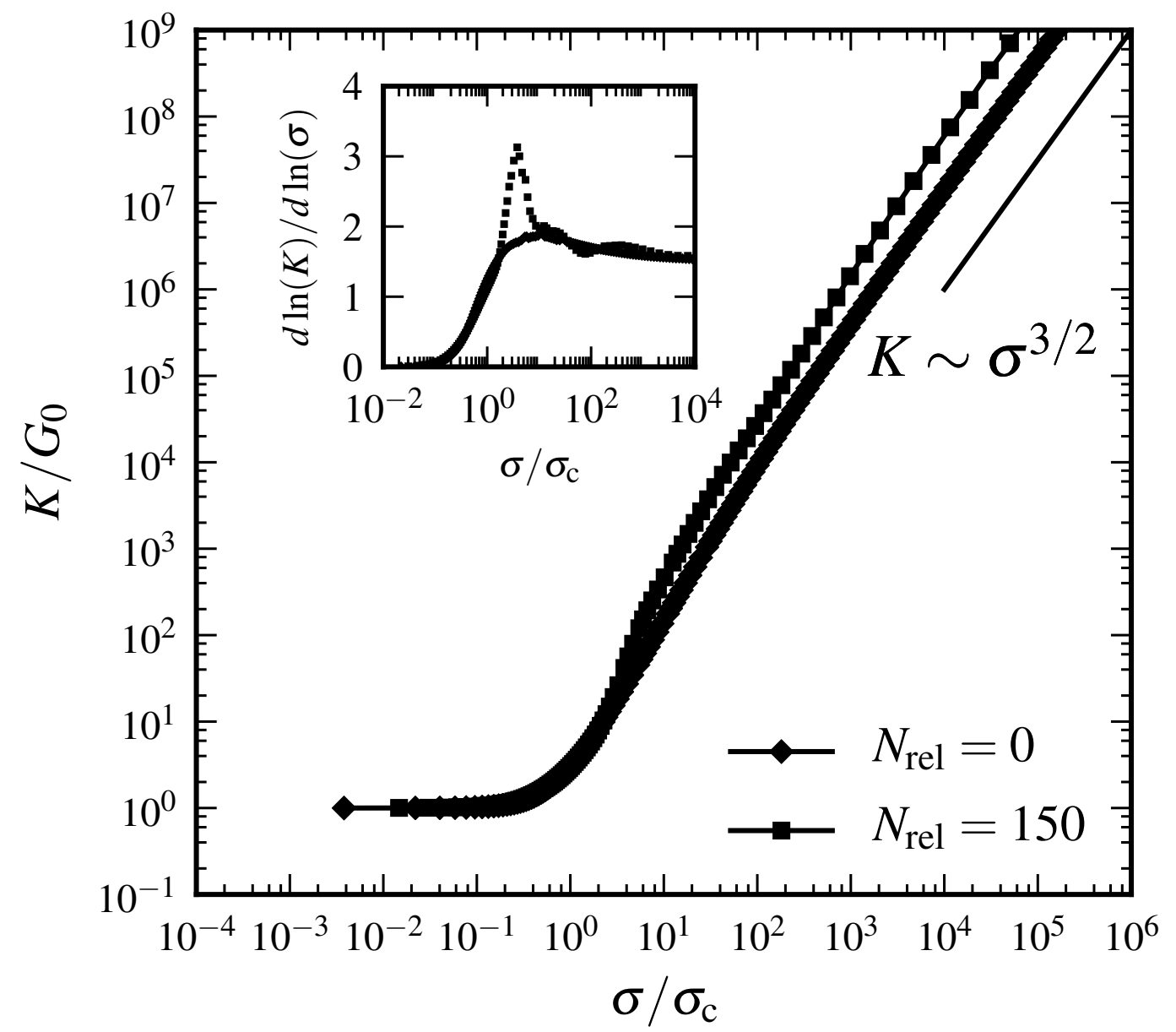

Figure 2.15.: Differential modulus as a function of shear stress, rescaled by linear modulus and critical stress $\sigma_{\mathrm{c}}=\sigma\left(\gamma_{\mathrm{c}}\right)$, respectively. Parameters: $N=3000, n=60$, $L=0.3, l_{0}=0.06, \alpha=0.5$, with $\left(N_{\text {rel }}=150\right)$ and without $\left(N_{\text {rel }}=0\right)$ relaxation. Inset shows the local slope $d \ln (K) / d \ln (\sigma)$ from the main plot. For large stresses, we observe power law scaling $K \sim \sigma^{3 / 2}$ (solid straight line). For intermediate stresses we recover slopes in the range of those derived from affine theory. 


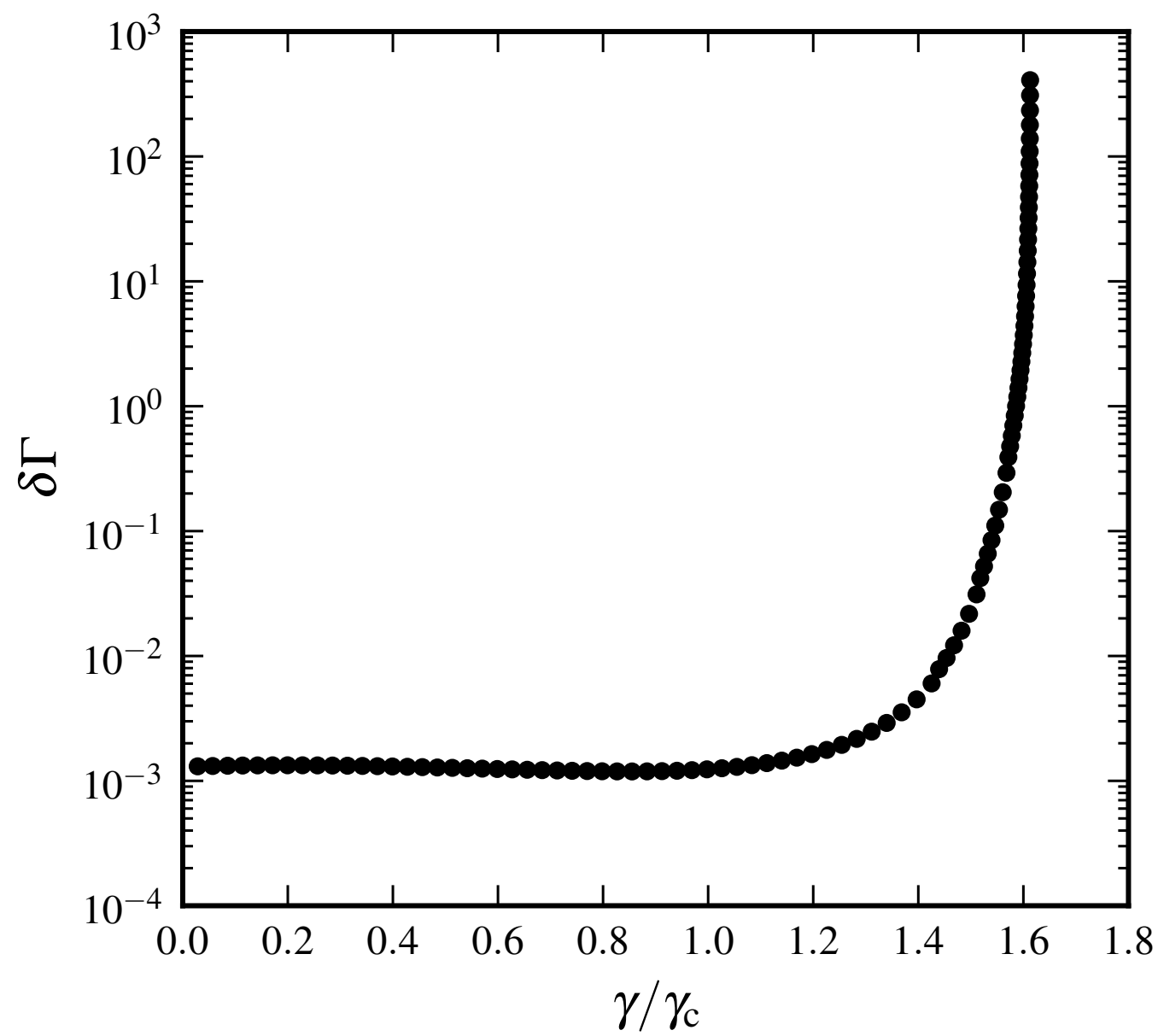

Figure 2.16.: Differential nonaffinity $\delta \Gamma$ as a function of scaled shear strain $\gamma / \gamma_{\mathrm{c}}$ for a system with $N=3000, n=60, L=0.3, l_{0}=0.06, \alpha=0.05$. 
where $\delta r_{\text {aff }}$ and $\delta r$ are the 3D coordinates of a filament's center of mass after applying an incremental shear strain $\delta \gamma$ without and with relaxation, respectively.

We let $\delta \Gamma$ denote the average of the differential nonaffinities over all filaments. Affine approximations imply $\delta \Gamma=0$. Fig. 2.16 shows that center of mass deformations are mostly affine for small strains. However, the differential nonaffinity increases starting at a strain around $\gamma_{\mathrm{c}}$ and eventually diverges as $\gamma \rightarrow \gamma_{\mathrm{d}}$. This can be understood, since the networks are strain stiffening, such that small incremental strain can induce large increase in the forces of individual crosslinks, thereby inducing large local rearrangements during energy minimization.

While increasing shear strain, there are force chains [36 38] developing in the network, which carry most of the tension, and which cannot reduce their strain due to the fact that they span the entire system (see inset of Fig. 2.17). We quantify this effect by considering tension profiles along filaments. The tension $\tau$ at position $x$ along a filament is given via $\tau(x)=\sum_{\left|x_{i}\right|>|x|} f_{\mathrm{cl}}\left(u_{i}\right)$, where $\left\{x_{i}\right\}$ are the crosslink binding sites and $\left\{u_{i}\right\}$ their extensions $\left(u_{i}=\epsilon x_{i}\right.$ in affine theory). Fig. 2.17 shows tension profiles averaged over all filaments for both, theoretical and simulated systems at various strains. In the simulations there is non-zero tension at zero strain due to prestress. With increasing $\gamma$, the simulations resemble the profiles expected from affine theory. However, when approaching the maximum strain $\gamma_{\mathrm{d}}$, the emergence of selective paths (force chains) that carry most of the tension becomes evident. The highly stretched crosslinks dominate the averaged tension profiles and therefore lead to jumps in the tension curves at the respective binding sites along the filament (green solid curve in Fig. 2.17).

\section{Bending}

Thus far we have restricted our theory and simulations to rigid filaments that can neither bend nor stretch. In Ref. [19], the authors considered finite stretching compliance of filaments, while bending compliance was assumed to be zero. They report that finite stretching stiffness does not impact the nonlinear stiffening regime of a composite network apart from the expected convergence (to some constant value) of the modulus at high strains. Here we complement this analysis by considering filaments that have finite bending but no stretching compliance. We performed simulations on a $2 \mathrm{D}$ network because of the relative computational ease compared to the $3 \mathrm{D}$ case. In addition to the energy stored in the crosslinks, we consider bending energy of the form $E_{\mathrm{b}}=\kappa \theta^{2} /\left(2 l_{\mathrm{av}}\right)$, where $\kappa$ is the bending rigidity, $\theta$ is the angle 


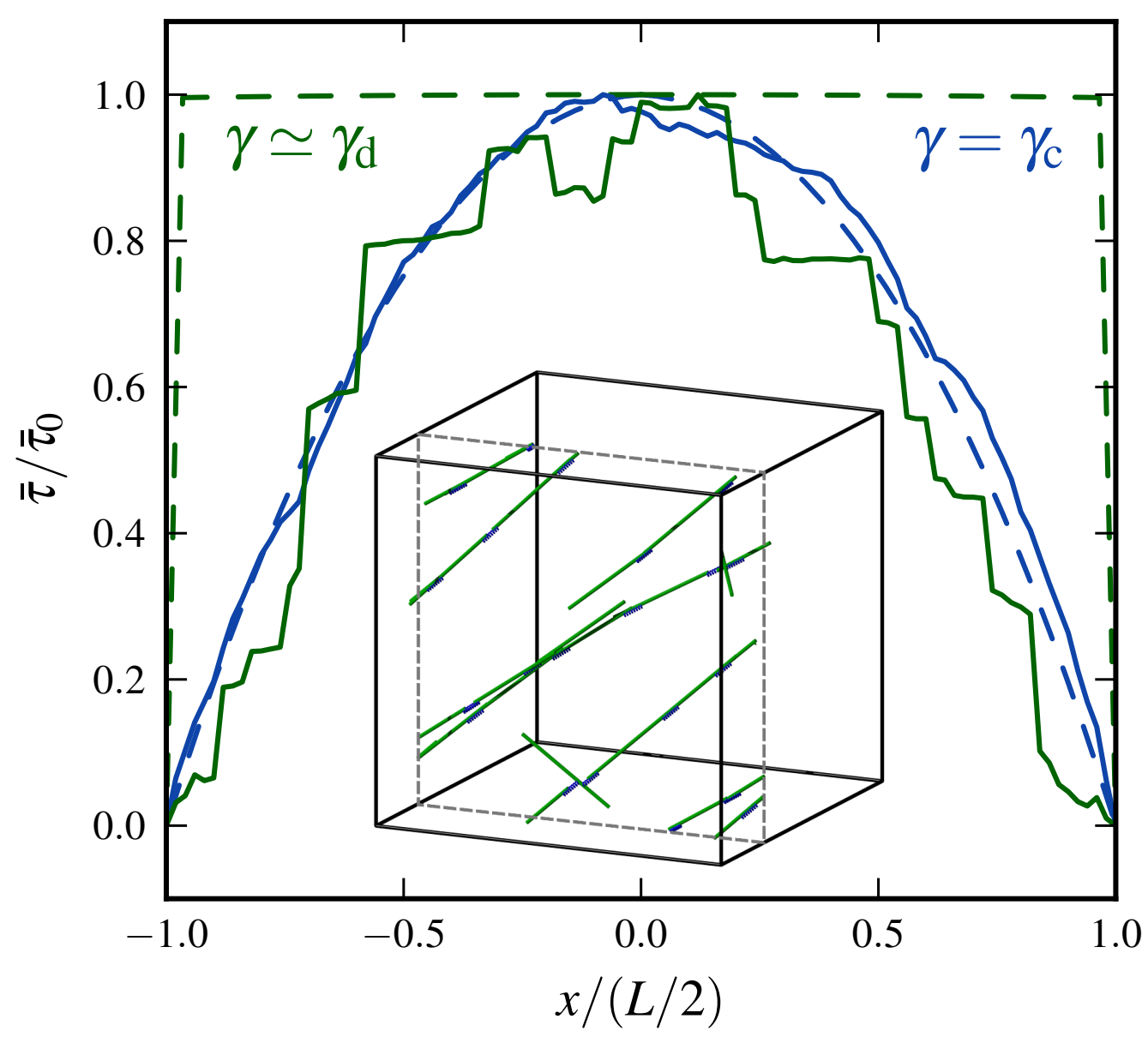

Figure 2.17.: Average tension $\bar{\tau}$ as a function of position $x$ along the filament for various strain values. Tension $\bar{\tau}$ is normalized by its maximum absolute value $\bar{\tau}_{0}$. Dashed curves correspond to theoretical results for $n=60$ at $\gamma=\gamma_{\mathrm{c}}$ (blue), $\gamma \simeq \gamma_{\mathrm{d}}$ (green). Solid curves show simulation data, with $N=3000, n=60, L=0.3$, $l_{0}=0.06, \alpha=0.5$. Inset shows a snapshot of the same system at maximum strain $\gamma_{\mathrm{d}} \simeq 0.6$ where only the 15 most stretched crosslinks and the corresponding filaments are shown. They form singular paths that span the whole system, thereby preventing further stress reduction via nonaffine rearrangements in these finite systems.

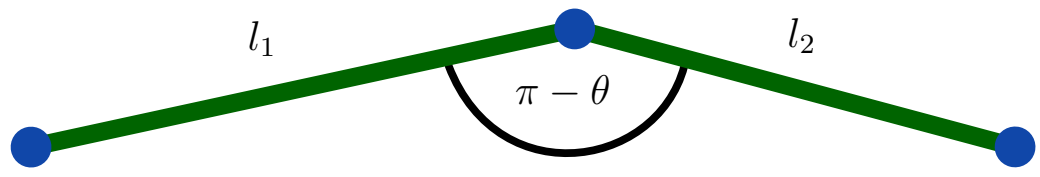

Figure 2.18.: Sketch of the local bending geometry of a filament (green) with crosslinks attached (blue). The local bending energy is given by $E_{\mathrm{b}}=\kappa \theta^{2} /\left(2 l_{\mathrm{av}}\right)$, with $\kappa$ being the bending rigidity and $l_{\mathrm{av}}=\left(l_{1}+l_{2}\right) / 2$. 
through which the filaments bend locally, and $l_{\mathrm{av}}=\left(l_{1}+l_{2}\right) / 2$ is the average distance between two adjacent pairs of crosslinks. We show the results in Fig. 2.19. The range of bending rigidity was chosen such that the linear modulus was still determined by the soft stretching modes of the crosslinks, so that bending did not impact the linear regime. As can be seen from these plots, bending compliance does not impact the nonlinear stiffening regime either - since bending modes are geometrically prohibited for large strains.

Thus, in isolation, neither bending nor stretching compliance of filaments impacts the nonlinear stiffening regime of composite networks. These findings suggest that the theoretical models at present cannot explain the $K \sim \sigma$ scaling observed in experiments.

\subsubsection{Conclusions}

We have studied the elastic properties of composite crosslinked filamentous networks in 3D analytically and numerically. We modeled such networks as a collection of rigid filaments connected by WLC crosslinks.

Based on the affine theory introduced in Ref. [19] we derived asymptotic power law scaling exponents for the differential elastic modulus with stress, in the limit of infinite crosslink density. In this case, the scaling exponents depend on the dimensionality of the system. In particular, we showed that 3D systems no longer exhibit a power law. Furthermore, we showed that for finite crosslink densities, the only persistent regime (over several orders of magnitude of stress) is the $\sigma^{3 / 2}$ scaling, as it is derived from the single WLC force-extension relation Eq. (2.1). This is in sharp contrast with the model proposed in Ref. [16, 26], where linear scaling was suggested, independent of the dimensionality of the system. This model implies finite stress at any strain and therefore does not apply to composite networks of rigid filaments with flexible crosslinks of finite length.

We further developed a simulation framework that allows us to measure the elastic response of random filamentous networks with WLC crosslinks. One important property of these 3D networks is that, by construction, they are prestressed due to initial extensions of the crosslinks. In addition to geometrical constraints, active elements such as motors can induce prestress as well [39]. We showed that the prestress in a network can dominate the linear response and might therefore be a feature that is worthwhile analyzing in experimental systems. 


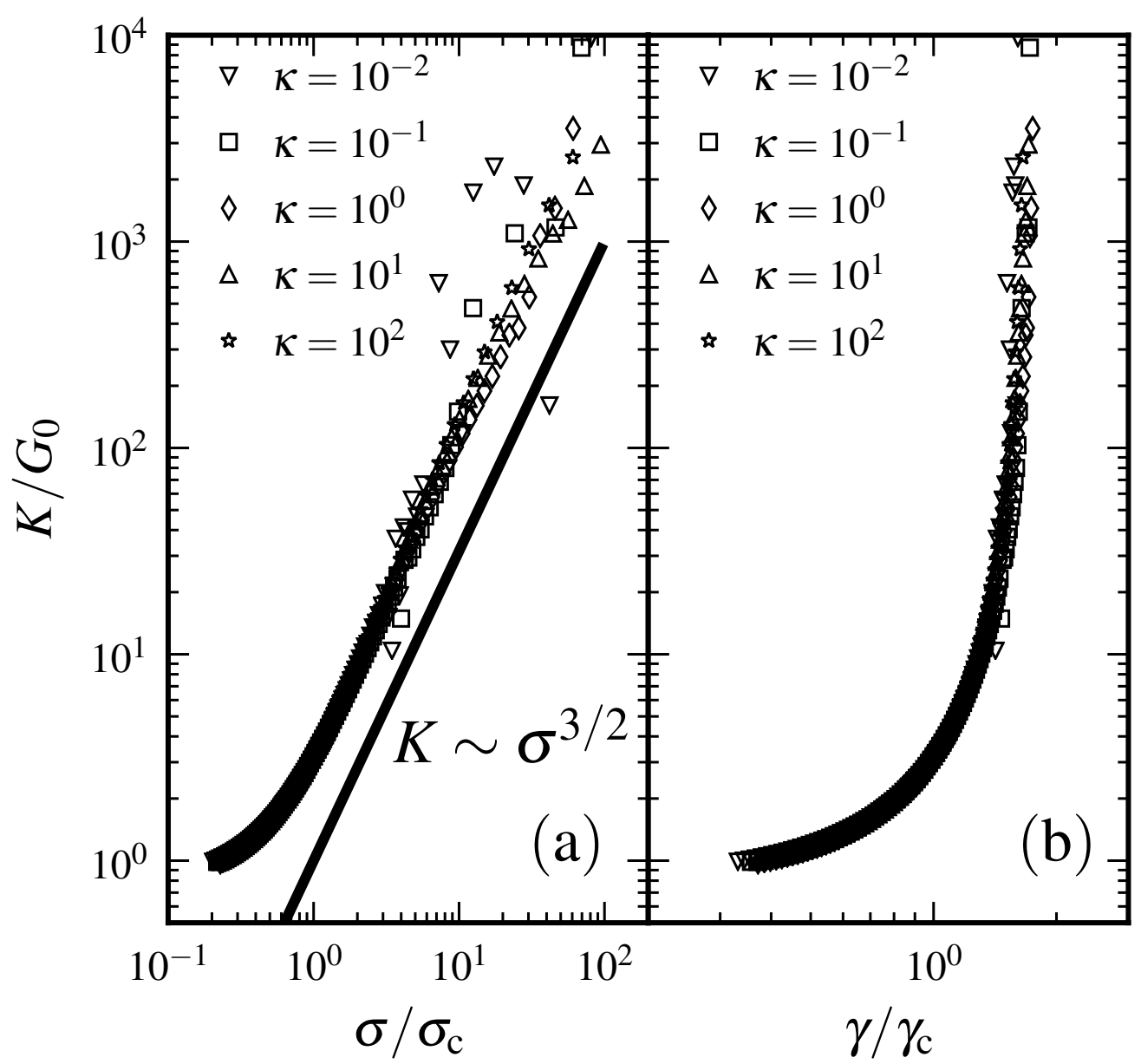

Figure 2.19.: (a) Differential modulus $K$ as a function of shear stress $\sigma$, rescaled by linear modulus $G_{0}$ and critical stress $\sigma_{c}=\sigma\left(\gamma_{c}\right)$, respectively, for various bending rigidities $\kappa$. Solid straight line indicates power law scaling $K \sim \sigma^{3 / 2}$. (b) Differential modulus $K$ as a function of shear strain $\gamma$, rescaled by linear modulus $G_{0}$ and critical strain $\gamma_{\mathrm{c}}$, respectively. Parameters: $N=800, L=1, l_{0}=0.1$, system-size $L_{x}=L_{y}=6$. 
Regarding nonlinear response, we observed divergence of stress (and differential modulus) at finite strain. Close to this strain we measured a power law scaling of the differential modulus with stress, with an exponent $3 / 2$, just as expected for a single WLC. In an intermediate-stress regime we observed local exponents that span the entire range of theoretically derived values for systems of differing dimensionality. The fact that our simulation results do not always resemble the predictions of a 3D affine theory, in this intermediate regime, may be attributed to nonaffine deformations. Extracting the exact set of assumptions - such as uniform crosslink density, isotropy, or zero prestress - that are responsible for these discrepancies is left for future investigation.

Experiments (see, e.g., 13, 20, 21]) have shown that in the nonlinear regime the differential modulus scales approximately linearly with the shear stress. We did not find such a regime in our simulations - neither when working with rigid filaments nor when incorporating finite bending stiffness (or enthalpic stretching as done in Ref. [19]). Therefore, we argue that none of the currently available theories can adequately explain the linear scaling of the differential modulus observed experimentally. It could possibly be that the WLC model does not accurately describe the elastic response of a single crosslink throughout the whole experimentally accessible regime. We speculate, however, that the linear scaling might be due to thermal fluctuations of the filaments, which have not been considered so far.

\subsubsection{Acknowledgements}

The authors would like to thank Fred MacKintosh for fruitful discussions. This work was funded by the Deutsche Forschungsgemeinschaft (DFG) within the collaborative research center SFB 755, project A3.

\subsubsection{Appendix: Derivation of scaling relations for the shear modulus}

\section{D network}

The integral Eq. 2.3 for the total energy of a single filament can be solved to give

$$
E_{1 \mathrm{D}}(\epsilon)=2 \frac{n}{L}\left[\frac{L^{3} \epsilon^{2}}{48 l_{0}}-\frac{L^{2} \epsilon}{32}-\frac{l_{0} L}{8}-\frac{l_{0}^{2}}{4 \epsilon} \ln \left(1-\frac{\epsilon L}{2 l_{0}}\right)\right]
$$


The divergence of the energy for $\epsilon \rightarrow \epsilon_{\mathrm{d}}=2 l_{0} / L$ stems from the term $\sim \frac{1}{\epsilon} \ln \left(1-\frac{\epsilon}{\epsilon_{\mathrm{d}}}\right)$, which is therefore the only one that we need to consider for the asymptotic scaling analysis in 2D and 3D.

\section{D network}

To approximate the solution of the integral in Eq. 2.12 we first carry out the $\phi$ integration analytically and obtain

$$
\begin{aligned}
\left\langle\sigma_{3 \mathrm{D}}\right\rangle_{\theta, \phi}(\gamma) & \sim \int_{0}^{\pi / 2} \frac{\arctan \left[\sqrt{\frac{1+\left(\gamma / \gamma_{\mathrm{d}}\right) \sin 2 \theta}{1-\left(\gamma / \gamma_{\mathrm{d}}\right) \sin 2 \theta}}\right]}{\sqrt{1-\left(\gamma / \gamma_{\mathrm{d}}\right)^{2} \sin ^{2} 2 \theta}} \\
& \times \sin \theta d \theta .
\end{aligned}
$$

The integral diverges for $\gamma=\gamma_{\mathrm{d}}$ due to a pole at $\theta=\pi / 4$. We can approximately consider $\tan ^{-1}\left[\sqrt{\frac{1+\left(\gamma / \gamma_{\mathrm{d}}\right) \sin 2 \theta}{1-\left(\gamma / \gamma_{\mathrm{d}}\right) \sin 2 \theta}}\right] \times \sin \theta$ as a constant because it takes finite values around the pole. Since we are interested in the regime close to the divergence of the integrand, we expand $\sin ^{2} 2 \theta$ up to second order in $\nu:=\theta-\pi / 4$. We arrive at

$$
\int_{-\pi / 4}^{\pi / 4} \frac{d \nu}{\sqrt{1-\left(\gamma / \gamma_{d}\right)^{2}\left(1-4 \nu^{2}\right)}}
$$

Approximation errors close to the boundary of the interval of integration that are made by expanding $\sin ^{2} 2 \theta$ are negligible, regarding the asymptotics, because the integrand diverges right at the center of the interval. Now we define $\mu:=1-\gamma / \gamma_{\mathrm{d}}$ and drop all terms of higher than first order in $\mu$, since we are interested in the behavior close to $\gamma=\gamma_{\mathrm{d}}$. With $\eta^{2}:=4 \nu^{2}$ and $\delta:=2 \mu$, we obtain

$$
\int_{-\pi / 2}^{\pi / 2} \frac{d \eta}{\sqrt{\eta^{2}(1-\delta)+\delta}} .
$$

This can be integrated, with the diverging part being

$$
\begin{aligned}
& \left.\sim \ln \left(2 \sqrt{\eta^{2}(1-\delta)^{2}+\delta(1-\delta)}+2(1-\delta) \eta\right)\right|_{-\pi / 2} ^{\pi / 2} \\
& \sim-\ln \delta \\
& \sim-\ln \left(1-\gamma / \gamma_{\mathrm{d}}\right)
\end{aligned}
$$

which is what has been proposed in Section 2.2.2. 
2. Networks with rigid filaments and compliant crosslinks

\section{References}

1. P. A. Janmey, U. Euteneuer, P. Traub \& M. Schliwa. Viscoelastic properties of vimentin compared with other filamentous biopolymer networks. J. Cell Biol. 113, 155-60 (1991).

2. F. C. MacKintosh \& P. A. Janmey. Actin gels. Current Opinion in Solid State and Materials Science 2, 350-357 (1997).

3. J. Xu, D. Wirtz \& T. D. Pollard. Dynamic Cross-linking by alpha -Actinin Determines the Mechanical Properties of Actin Filament Networks. J. Biol. Chem. 273, 9570-9576 (1998).

4. M. L. Gardel et al. Elastic behavior of cross-linked and bundled actin networks. Science 304, 1301-5 (2004).

5. M. L. Gardel et al. Scaling of F-Actin Network Rheology to Probe Single Filament Elasticity and Dynamics. Phys. Rev. Lett. 93, 1-4 (2004).

6. C. Storm, J. J. Pastore, F. C. Mackintosh, T. C. Lubensky \& P. A. Janmey. Nonlinear elasticity in biological gels. Nature 435, 191-194 (2005).

7. A. R. Bausch \& K. Kroy. A bottom-up approach to cell mechanics. Nature Physics 2, 231-238 (2006).

8. G. H. Koenderink, M. Atakhorrami, F. C. MacKintosh \& C. F. Schmidt. HighFrequency Stress Relaxation in Semiflexible Polymer Solutions and Networks. Phys. Rev. Lett. 96, 138307 (2006).

9. O. Chaudhuri, S. H. Parekh \& D. A. Fletcher. Reversible stress softening of actin networks. Nature 445, 295-8 (2007).

10. P. A. Janmey et al. Negative normal stress in semiflexible biopolymer gels. Nature Materials 6, 48-51 (2007).

11. K. E. Kasza et al. The cell as a material. Curr. Opin. Cell Biol. 19, 101-7 (2007).

12. J. Liu, G. H. Koenderink, K. E. Kasza, F. C. MacKintosh \& D. A. Weitz. Visualizing the Strain Field in Semiflexible Polymer Networks: Strain Fluctuations and Nonlinear Rheology of F-Actin Gels. Phys. Rev. Lett. 98, 198304 (2007).

13. M. L. Gardel et al. Prestressed F-actin networks cross-linked by hinged filamins replicate mechanical properties of cells. Proc. Natl. Acad. Sci. U.S.A. 103, 1762-1767 (2006). 
14. M. L. Gardel et al. Stress-dependent elasticity of composite actin networks as a model for cell behavior. Phys. Rev. Lett. 96, 088102 (2006).

15. B. A. DiDonna \& A. J. Levine. Unfolding cross-linkers as rheology regulators in F-actin networks. Phys. Rev. E: Stat., Nonlinear, Soft Matter Phys. 75, 041909 (2007).

16. C. P. Broedersz, C. Storm \& F. C. MacKintosh. Nonlinear elasticity of composite networks of stiff biopolymers with flexible linkers. Phys. Rev. Lett. 101, 118103 (2008).

17. P. Dalhaimer, D. E. Discher \& T. C. Lubensky. Crosslinked actin networks show liquid crystal elastomer behaviour, including soft-mode elasticity. Nature Physics 3, 354-360 (2007).

18. H. Lee et al. Cytoskeletal deformation at high strains and the role of cross-link unfolding or unbinding. Cellular and Molecular Bioengineering 2, 28-38 (2009).

19. A. Sharma, M. Sheinman, K. M. Heidemann \& F. C. MacKintosh. Elastic response of filamentous networks with compliant crosslinks. Phys. Rev. E: Stat., Nonlinear, Soft Matter Phys. 88, 052705 (2013).

20. K. E. Kasza et al. Nonlinear elasticity of stiff biopolymers connected by flexible linkers. Phys. Rev. E: Stat., Nonlinear, Soft Matter Phys. 79, 041928 (2009).

21. K. E. Kasza et al. Actin filament length tunes elasticity of flexibly cross-linked actin networks. Biophys. J. 99, 1091-100 (2010).

22. C. Bustamante, J. F. Marko, E. D. Siggia \& S. Smith. Entropic elasticity of lambda-phage DNA. Science 265, 1599-1600 (1994).

23. J. F. Marko \& E. D. Siggia. Stretching DNA. Macromolecules 28, 8759-8770 (1995).

24. I. Schwaiger, A. Kardinal, M. Schleicher, A. A. Noegel \& M. Rief. A mechanical unfolding intermediate in an actin-crosslinking protein. Nature structural $\&$ molecular biology 11, 81-85 (2004).

25. S. Furuike, T. Ito \& M. Yamazaki. Mechanical unfolding of single filamin A (ABP-280) molecules detected by atomic force microscopy. FEBS letters 498, $72-75$ (2001). 
26. C. P. Broedersz, C. Storm \& F. C. MacKintosh. Effective-medium approach for stiff polymer networks with flexible cross-links. Phys. Rev. E: Stat., Nonlinear, Soft Matter Phys. 79, 61914 (2009).

27. L. Landau \& E. Lifshitz. Elasticity theory (Pergamon Press, 1975).

28. A. Wächter \& L. T. Biegler. On the implementation of an interior-point filter line-search algorithm for large-scale nonlinear programming. Math. Prog. 106, $25-57$ (2005).

29. A. W. Lees \& S. F. Edwards. The computer study of transport processes under extreme conditions. J. Phys. C: Solid State Phys. 5, 1921-1928 (1972).

30. J. Wilhelm \& E. Frey. Elasticity of Stiff Polymer Networks. Phys. Rev. Lett. 91, 108103 (2003).

31. D. A. Head, A. J. Levine \& F. C. MacKintosh. Deformation of Cross-Linked Semiflexible Polymer Networks. Phys. Rev. Lett. 91, 2-5 (2003).

32. P. R. Onck, T. Koeman, T. van Dillen \& E. van der Giessen. Alternative Explanation of Stiffening in Cross-Linked Semiflexible Networks. Phys. Rev. Lett. 95, 19-22 (2005).

33. R. Connelly \& A. Back. Mathematics and Tensegrity. American Scientist 86, 142 (1998).

34. A. Pugh. An introduction to tensegrity (University of California Pr, 1976).

35. S. Alexander. Amorphous solids: their structure, lattice dynamics and elasticity. Physics Reports 296, 65-236 (1998).

36. C. Heussinger \& E. Frey. Force distributions and force chains in random stiff fiber networks. The European Physical Journal E, 1-8 (2007).

37. E. M. Huisman, T. van Dillen, P. R. Onck \& E. Van der Giessen. ThreeDimensional Cross-Linked F-Actin Networks: Relation between Network Architecture and Mechanical Behavior. Phys. Rev. Lett. 99, 2-5 (2007).

38. G. Žagar, P. R. Onck \& E. Van der Giessen. Elasticity of Rigidly Cross-Linked Networks of Athermal Filaments. Macromolecules 44, 7026-7033 (2011).

39. G. H. Koenderink et al. An active biopolymer network controlled by molecular motors. Proc. Natl. Acad. Sci. U.S.A. 106, 15192-7 (2009). 


\section{Random spring networks on the unit circle}

This chapter contains the manuscript:

- K. M. Heidemann, A. O. Sageman-Furnas, A. Sharma, F. Rehfeldt, C. F. Schmidt \& M. Wardetzky. Random spring networks on the unit circle (in preparation)

We give a short summary of the key findings obtained within the corresponding study, before presenting the full manuscript.

\subsection{Key results}

In this study, we tackle the challenge of deriving an analytical theory for a network modeling approach that takes into account the full topology of a highly connected spring network (see Section 1.2.2). This is in contrast to our approach in Chapter 2 where we combine network simulations with an analytical continuum theory that neglects network connectivity. Instead of focusing on bulk elastic properties, we here aim at results concerning the force distribution within the network.

Compared to the 3D composite networks of rods and nonlinear springs with periodic boundary conditions studied in Chapter 2, we consider a simplified system. We construct linear spring networks on the unit circle. Each network consists of $N$ randomly positioned nodes that are randomly connected by linear springs with rest length zero, so that each node carries, on average, $z$ springs - where $z$ is referred to as the average degree (see Fig. 3.1). The signed spring lengths are measured along the circular arc between two nodes.

We formulate the problem of finding the network configuration in mechanical equilibrium given a particular network topology (a graph with $N$ nodes and average degree $z$ ), and initial spring lengths $\bar{l}$. This is equivalent to minimizing the energy 

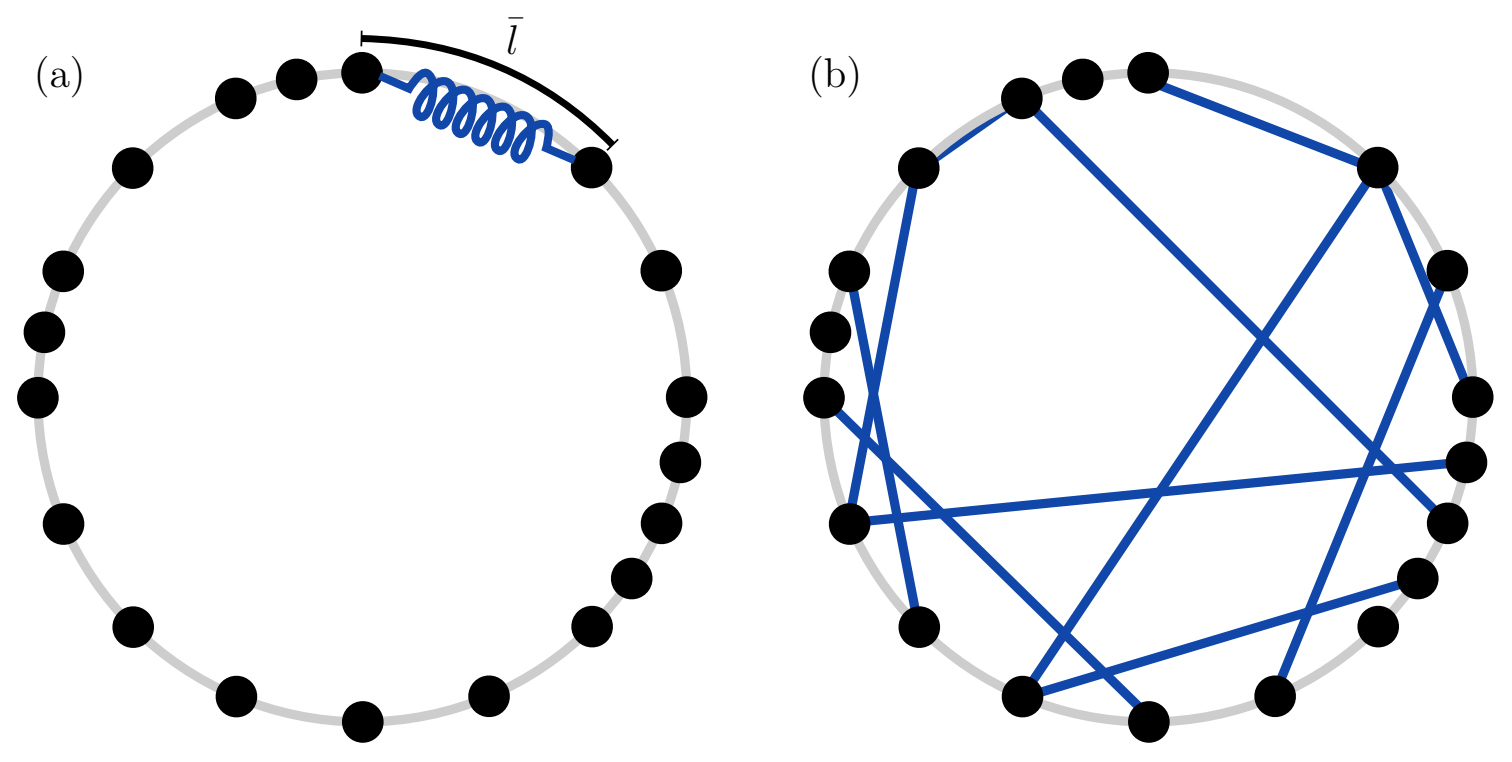

Figure 3.1.: Spring network generation on the unit circle: (a) Put $N$ nodes randomly on the circle. Then connect random node pairs with linear springs along the shortest connecting circular arc (initial length $\bar{l}$ ). (b) Put more and more springs (here represented by straight lines), until each node is on average connected to $z$ springs.

of the network. Importantly, there are additional constraints that guarantee that the network remains on the circle; in particular, they prevent contraction of cycles that wind around the circle (see Fig. 3.4, and Fig. 3.2 for a $2 \mathrm{D}$ visualization of contractibility). Otherwise, the entire network would collapse onto a single point. The resulting optimization problem is quadratic and therefore admits a unique explicit solution for the final spring lengths $\boldsymbol{l}^{*}$ (see Eq. (3.3)).

Instead of only looking at the relaxation of a particular network, we also consider ensembles, where we fix node number $N$ and average degree $z$ but not the initial spring lengths $\overline{\boldsymbol{l}}$ or the full graph representation of the network. By using graph theoretical considerations, we can derive the variance $\sigma_{\Delta l}^{2}$ of the expected length changes $\boldsymbol{\Delta} \boldsymbol{l}=\boldsymbol{l}^{*}-\overline{\boldsymbol{l}}$ upon relaxation. Surprisingly, it solely depends on $N$ and $z$ (see Table 3.1). At this point, we would like to stress that a mean field calculation, that only considers the springs directly attached to a node (see Fig. 3.3 for a schematic comparison of the mean field and graph theoretical approach), would lead to a different result for the variance $\sigma_{\Delta l}^{2}$. In particular, both show the same leading order term, but differ significantly for small $z$ (see Table 3.1). Hence, the inclusion of the entire network structure is essential for capturing the final length distribution in the network. 


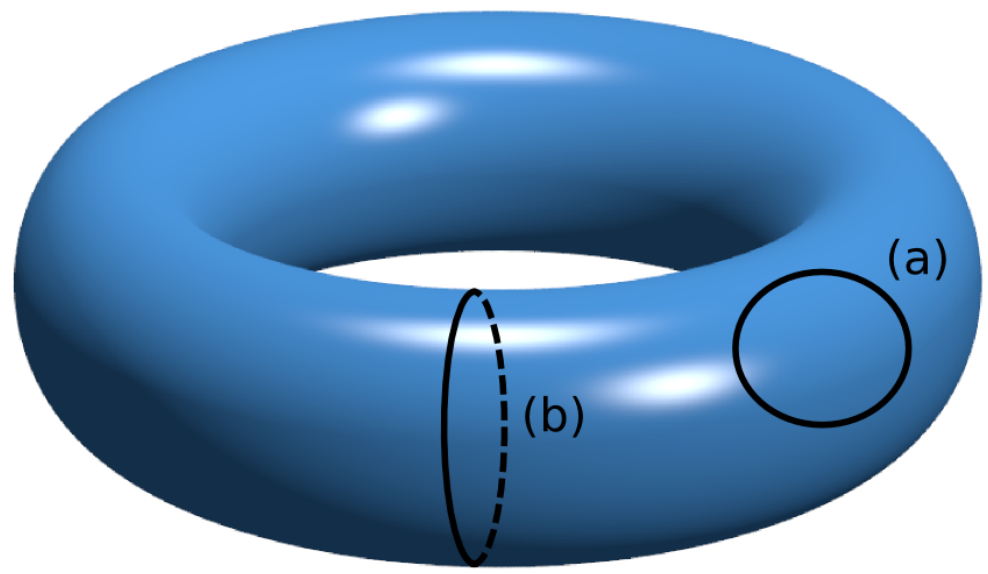

Figure 3.2.: Illustration of contractible (a) and noncontractible (b) loops/cycleshere, on the torus instead of the circle.

Table 3.1.: Variance of the distribution of spring length changes $\sigma_{\Delta l}^{2}$ for the graph theoretical approach compared to a mean field approach. $\sigma_{\bar{l}}^{2}$ corresponds to the variance of the initial length distribution.

\begin{tabular}{lll}
\hline & graph theory & mean field \\
\hline$\sigma_{\Delta l}^{2}$ & $\frac{2}{z}\left(1-\frac{1}{N}\right) \sigma_{\bar{l}}^{2}$ & $\frac{2}{z}\left(1+\frac{1}{z}\right) \sigma_{\bar{l}}^{2}$ \\
\hline
\end{tabular}

(a)

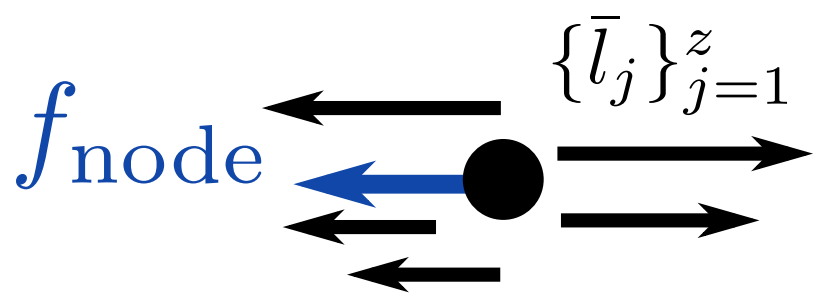

(b)

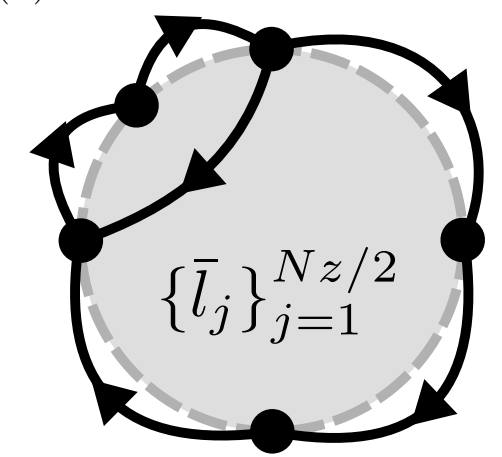

Figure 3.3.: (a) Mean field approach: Node displacements are assumed to be determined by the initial force $f_{\text {node }}$ acting on a node - given by the sum of spring lengths $\left\{\bar{l}_{j}\right\}_{j=1}^{z}$ that are attached to it. The remaining springs in the network are not taken into account. (b) Graph theoretical approach: Changes in spring lengths depend on the entire network, i.e., the graph associated with the network, together with the set of initial spring lengths $\left\{\bar{l}_{j}\right\}_{j=1}^{N z / 2}$. 
3. Random spring networks on the unit circle

Finally, we consider the full final spring length distribution $p_{l^{*}}(l)$ in the case $2<z \ll N$. We derive a closed-form expression that is in excellent agreement with numerical simulations. 


\title{
3.2. Manuscript
}

\begin{abstract}
We study the distribution of forces in random spring networks on the unit circle using a combination of probabilistic theory and numerical computation. An initially generated network with internal forces relaxes into mechanical equilibrium. Using graph theory, we derive mean and variance of the final length distribution, which depend on topological parameters like node number and average degree of the network, but not on the explicit graph representation. We show that an estimate for the expected distribution can be obtained analytically. We demonstrate the accuracy of our approach by comparing with numerics. Our analytical approach is straightforwardly generalized to higher spatial dimensions.
\end{abstract}

\subsubsection{Introduction}

Disordered filamentous networks are ubiquitous in nature. They are found at the intracellular level as in the cytoskeleton of animal cells and at the extracellular level in tissues. Under loading, these networks determine the mechanical response by generating elastic forces on a macroscopic scale. Due to the filamentous nature of these networks, the strain field, and hence the force distribution within the network, can be highly inhomogeneous [1, 2]. Large local stresses can also result due to contractile forces within a network, for instance due to molecular motors [3 5] or platelets in blood clot [6, 7]. The broad distribution of forces makes a network susceptible to internal rearrangements as local stresses can result in filaments getting disconnected from each other due to unbinding of crosslinking proteins [5, 8, 9] or undergoing local rupture [10]. The loss of local connectivity due to stresses is manifested as gradual loss of stiffness.

Quantitative analysis of the force distribution within a random disordered network has primarily relied on computational modeling of such networks [1. This is due to the fact that the network deformation is highly nonaffine on the microscopic scale [11-13] making an analytical treatment of the deformation field difficult. Even under no loading conditions, a network can be internally stressed [14] resulting in a broad distribution of forces.

In this study, we attempt a very general, statistical and analytical analysis of force distributions in random disordered networks. Our model system is a random spring network, which has been widely used in previous studies to model the elastic behavior 


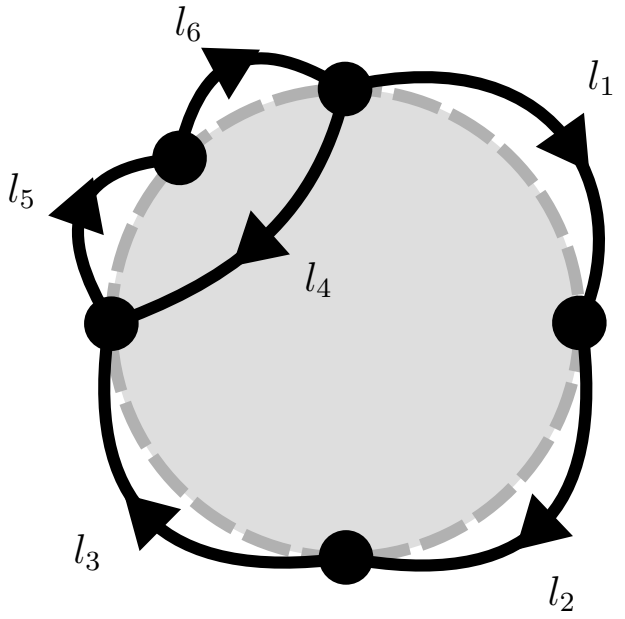

(a)

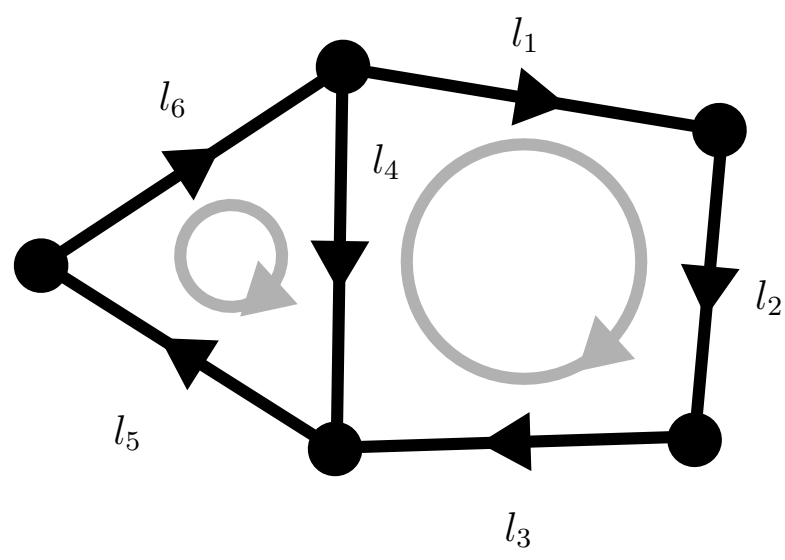

(b)

Figure 3.4.: (a) Small example network embedded on the circle. (b) Graph representation of the network in (a). The edge/spring orientations are depicted via the black arrows. The network contains two independent (fundamental) cycles: $\left\{l_{1}, l_{2}, l_{3}, l_{4}\right\}$ and $\left\{l_{4}, l_{5}, l_{6}\right\}$. After choosing arbitrary walking directions for both cycles (grey arrows), we can construct the linear constraints that fix cycle contractibility (see Eq. (3.2)) - here: $l_{1}+l_{2}+l_{3}-l_{4}=1$ (winds clockwise around circle) and $l_{4}+l_{5}+l_{6}=0$ (contractible).

of biological networks. We consider random spring networks with internal forces such that the network, in its initial configuration, is not in mechanical equilibrium. Using a statistical and analytical approach we describe the force distribution in a random spring network once it attains mechanical equilibrium. We also perform numerical simulations and show that our analytics are in very good agreement with the numerics.

\subsubsection{Model and simulations}

We define a one-dimensional circular spring network to be a collection of nodes on a circle connected by linear angular springs with rest length zero (see Fig. 3.4).

We construct an initial network configuration as follows: (i) Draw $N$ node positions uniformly on the circle. (ii) Draw $N z / 2$ node pairs randomly and connect them via springs. We choose as degrees of freedom the signed spring lengths in units of the circle's circumference (positive sign means that nodes are connected clockwise and vice versa). Initially, we always connect via the shortest distance along the circle. The network can be readily encoded within a graph representation - the springs being 
the directed edges of the graph, with lengths as edge weights. We either connect nodes randomly, i.e., create a random graph with average degree $z$ or set up a regular graph with constant degree $z$.

The task is to find a mathematical description for the problem of minimizing the total elastic energy of such a network, i.e., to find

$$
\mathbf{l}^{*}:=\underset{\boldsymbol{l}}{\arg \min } \frac{1}{2} \boldsymbol{l}^{T} \boldsymbol{l},
$$

where $\mathbf{l} \in \mathbb{R}^{N z / 2}$ is the vector of all spring lengths. However, the network being embedded onto the circle imposes additional boundary conditions. Each cycle in the graph may either be contractible or not, meaning that it winds around the circle at least once (see Fig. 3.4). In order to fix the cycle contractibility of the network we first determine the $m=N(z / 2-1)+1$ fundamental cycles ${ }^{1}$ of the undirected version of the graph. Then we walk along every fundamental cycle according to a chosen orientation and sum up the initial lengths of the springs that are part of it. We need to take the negative spring length if we walk against the edge direction of the directed graph. For each cycle the summation results in an integer number (winding number), where 0 corresponds to a contractible cycle, -1 to a cycle, which has been walked counterclockwise, and 1 clockwise, etc. (see Fig. 3.4 for an example). This allows us to set up a system of linear equations:

$$
\mathrm{Cl}=\mathrm{g}=\mathrm{C} \bar{l} .
$$

We call $\mathbf{g} \in \mathbb{Z}^{m}$ the vector of winding numbers. It is determined by the vector of initial spring lengths $\overline{\mathbf{l}}$ and the signed cycle matrix $\mathbf{C} \in \mathbb{Z}^{m \times N z / 2}$. The entries $C_{i j}$ are 1 or -1 if spring $j$ is part of the $i$ th fundamental cycle and 0 otherwise (see explanation above).

Together, Eqs. (3.1) and (3.2) define a quadratic programming problem with unique analytic solution

$$
\boldsymbol{l}^{*}=\mathbf{C}^{T}\left(\mathbf{C} \mathbf{C}^{T}\right)^{-1} \mathbf{C} \overline{\boldsymbol{l}}=: \mathbf{P} \overline{\boldsymbol{l}},
$$

which can be explicitly computed via, e.g., the optimization library IPOPT [15].

\footnotetext{
${ }^{1} \mathrm{~A}$ fundamental cycle is defined as a cycle that occurs when adding a single edge to a spanning tree of the graph. There are $N-1$ edges in the spanning tree, so $N z / 2-(N-1)$ edges can be added. Hence, there are $N(z / 2-1)+1$ fundamental cycles.
} 


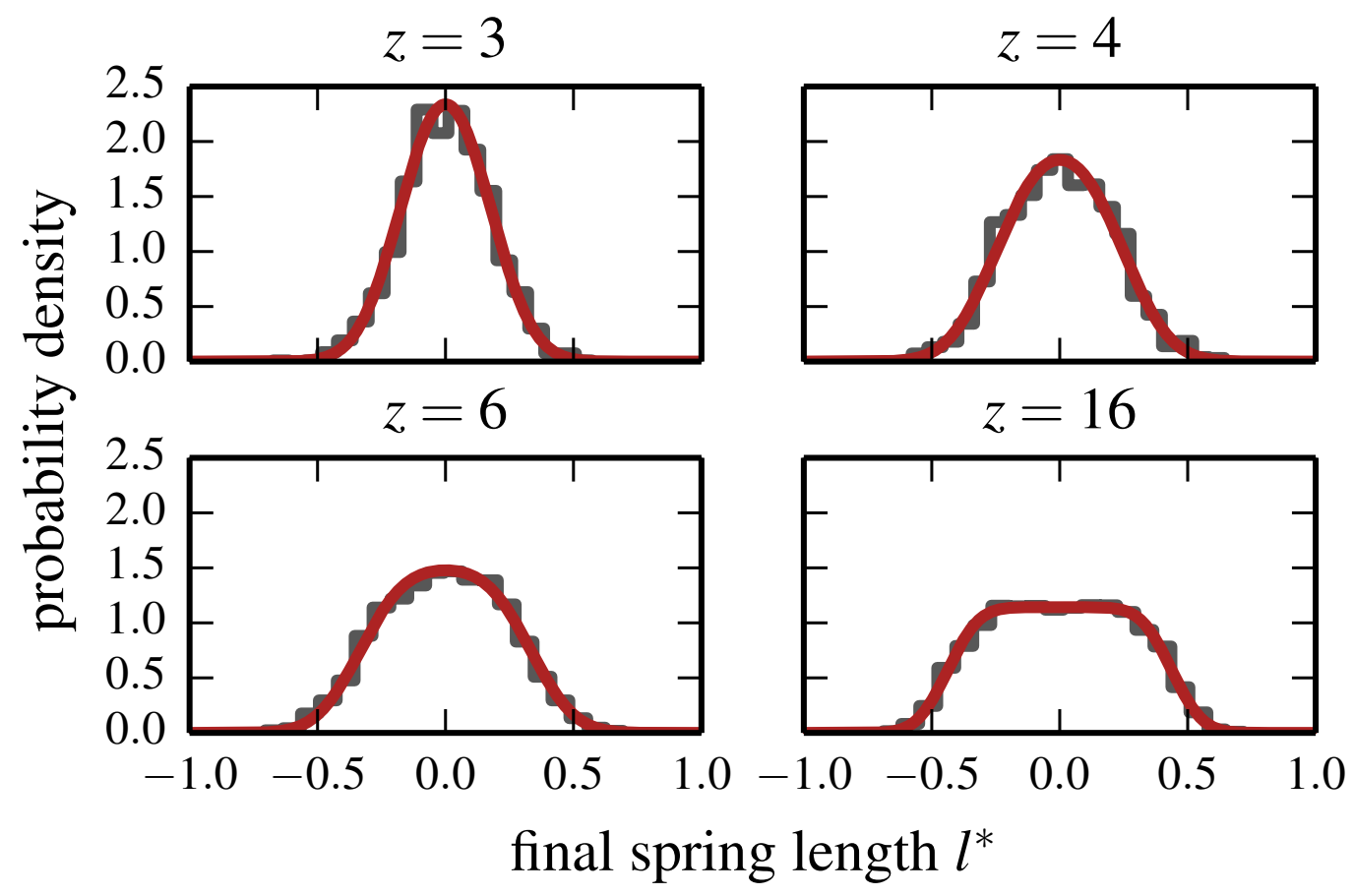

Figure 3.5.: Comparison between the expected final spring length distribution Eq. (3.21) (solid red curve) and simulations (grey histogram) for individual random regular spring networks with $N=1000$ nodes and degree $z=\{3,4,6,16\}$.

\subsubsection{Probabilistic theory}

Instead of solving a specific realization of a network via Eq. (3.3), which requires knowledge of the entire graph (encoded in $\mathbf{C}$ ), we here establish a probabilistic theory that relates the expected final distribution of spring lengths to essentially two topological parameters: (i) number of nodes $N$, and (ii) degree $z$ (see Fig. 3.5 for a comparison of the two approaches).

We first need to find a formal representation for the distributions of spring lengths. For a particular realization of any length vector $\boldsymbol{l}$ (can be the initial, $\overline{\boldsymbol{l}}$, or final, $\boldsymbol{l}^{*}$, etc.), the corresponding cumulative histogram $H_{l}$ is given via

$$
H_{l}(x)=\frac{2}{N z} \sum_{i=1}^{N z / 2} \mathbf{1}_{l_{i} \leq x}
$$

where $\mathbf{1}_{A}$ is the indicator function (one if $A$ is true, zero otherwise). It measures the number of elements in $\boldsymbol{l}$ with values smaller or equal to $x$. The smooth distribution 


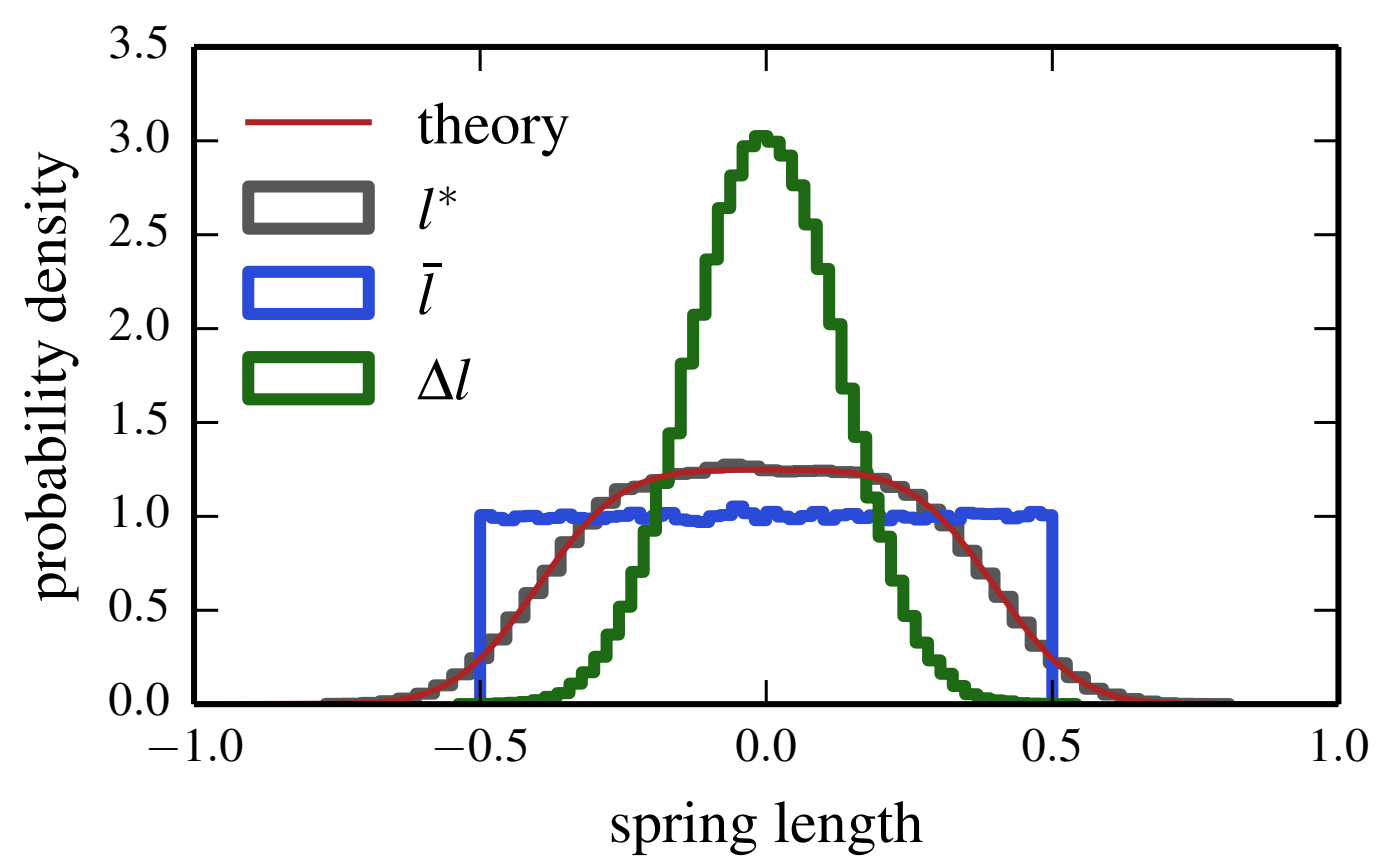

Figure 3.6.: Histograms $p_{\boldsymbol{l}^{*}}, p_{\bar{l}}$, and $p_{\boldsymbol{\Delta} \boldsymbol{l}}$ for an ensemble average over 50 simulations of random networks with $N=1000$ nodes and average degree $z=10$. The red solid line corresponds to the theoretical density $p_{l^{*}}$ (Eq. (3.21)).

function $F_{l}$, which is approached in the limit of many repeated experiments, is given via the expected value:

$$
\begin{aligned}
F_{l}(x) & :=\mathrm{E}\left[H_{l}(x)\right]=\frac{2}{N z} \sum_{i=1}^{N z / 2} \mathrm{E}\left[\mathbf{1}_{l_{i} \leq x}\right] \\
& =\frac{2}{N z} \sum_{i=1}^{N z / 2} \mathrm{P}\left(l_{i} \leq x\right)=\frac{2}{N z} \sum_{i=1}^{N z / 2} F_{l_{i}}(x),
\end{aligned}
$$

which is the average over the marginal distribution functions of the individual $l_{i}$. This result translates directly into the corresponding probability densities, i.e.,

$$
p_{l}(x)=\frac{d}{d x} F_{l}(x)=\frac{2}{N z} \sum_{i=1}^{N z / 2} p_{l_{i}}(x) .
$$

In order to derive the probability density $p_{l^{*}}$ for the final spring lengths, we look at the displacements from the initial configuration, i.e., write $\boldsymbol{l}^{*}=\overline{\boldsymbol{l}}+\boldsymbol{\Delta} \boldsymbol{l}$, where $\boldsymbol{\Delta} \boldsymbol{l}$ 
are the changes in spring lengths upon relaxation into mechanical equilibrium. The probability density is then given as

$$
\begin{aligned}
p_{\boldsymbol{l}^{*}}(x) & =p_{\overline{\boldsymbol{l}}+\boldsymbol{\Delta} \boldsymbol{l}}(x)=\int_{-\infty}^{+\infty} p_{\overline{\boldsymbol{l}}, \boldsymbol{\Delta} \boldsymbol{l}}(a, x-a) d a \\
& =\int_{-\infty}^{+\infty} p_{\overline{\boldsymbol{l}}}(a) \cdot p_{\boldsymbol{\Delta} \boldsymbol{l} \mid \bar{l}}(x-a) d a,
\end{aligned}
$$

leading to the density $p_{l^{*}}$ for the full final spring vector via Eq. (3.6). We assume all $p_{\bar{l}_{i}}$ to be identical and having zero mean, i.e., $\mu_{\bar{l}_{i}}=0$. For the model described before this is true, in particular,

$$
p_{\bar{l}_{i}}=\mathcal{U}(-0.5,0.5)
$$

because the initial node positions are uniformly distributed on the circle. Hence, we still need to derive the conditional probability density for the spring length changes $p_{\Delta l_{i} \mid \bar{l}_{i}}$.

Reconsidering Eq. (3.3), we can write

$$
\begin{aligned}
\Delta \boldsymbol{l} & =\boldsymbol{l}^{*}-\overline{\boldsymbol{l}}=(\mathbf{P}-\mathbf{I}) \overline{\boldsymbol{l}}=: \mathbf{S} \overline{\boldsymbol{l}} \\
\Rightarrow \Delta l_{i} & =\sum_{j=1}^{N z / 2} S_{i j} \bar{l}_{j} .
\end{aligned}
$$

The $\bar{l}_{j}$ are not independent since they are coupled through the cycle constraints (see Eq. (3.2p). It is therefore challenging to access $p_{\Delta l \mid \bar{l}}$ directly. Instead, we consider its moments, i.e., $\mu_{\Delta l \mid \bar{l}}$ and $\sigma_{\Delta l \mid \bar{l}}^{2}$. In particular, Eq. 3.11 implies $\mathrm{E}\left(\Delta l_{i} \mid \bar{l}_{i}=\bar{l}\right)=S_{i i} \bar{l}$, and therefore with Eq. (3.6):

$$
\begin{aligned}
\mu_{\Delta l \mid \bar{l}} & =\frac{2}{N z} \sum_{i=1}^{N z / 2} \mathrm{E}\left(\Delta l_{i} \mid \bar{l}_{i}=\bar{l}\right) \\
& =\frac{2}{N z} \sum_{i=1}^{N z / 2} S_{i i} \bar{l}=\frac{2 \bar{l}}{N z} \operatorname{tr} \mathbf{S} .
\end{aligned}
$$

We make use of our knowledge about the graph's (directed) cycle matrix $\mathbf{C}$ to determine $\operatorname{tr} \mathbf{S}$. First, note that by definition $\operatorname{tr} \mathbf{S}=\operatorname{tr} \mathbf{P}-N z / 2$ (see Eq. (3.10)). The projector property of $\mathbf{P}$ (i.e., $\mathbf{P}^{2}=\mathbf{P}$ ) leads to $\operatorname{tr} \mathbf{P}=\operatorname{dim}(\operatorname{Im} \mathbf{P})=N z / 2-\operatorname{dim}(\operatorname{ker} \mathbf{P})$, 
because $\mathbf{P}$ has eigenvalues 0 and 1 only. Furthermore, ker $\mathbf{P}=\operatorname{ker} \mathbf{C}$, by definition (see Eq. (3.3) ) and hence $\operatorname{tr} \mathbf{S}=-\operatorname{dim}(\operatorname{ker} \mathbf{C})$. Recall that $\mathbf{C}$ contains $N(z / 2-1)+1$ linearly independent rows corresponding to a set of fundamental cycles of the graph, i.e., has full rank and thereby $\operatorname{dim}(\operatorname{ker} \mathbf{C})=N z / 2-(N(z / 2-1)+1)=N-1$. It follows that

$$
\operatorname{tr} \mathbf{S}=1-N
$$

Surprisingly, tr $\mathbf{S}$ does only depend on the number of nodes in the graph, not on their degrees. Substituting in Eq. (3.12) yields:

$$
\mu_{\Delta l \mid \bar{l}}=-\frac{2 \bar{l}}{z}\left(1-\frac{1}{N}\right) .
$$

The conditional variance $\sigma_{\Delta l \mid \bar{l}}^{2}$ cannot be expressed simply as a function of $N$ and $z$. However, the law of total variance [16] allows for calculation of the expected variance:

$$
\mathrm{E}_{\bar{l}}[\operatorname{Var}(\boldsymbol{\Delta} \boldsymbol{l} \mid \overline{\boldsymbol{l}})]=\operatorname{Var}(\boldsymbol{\Delta} \boldsymbol{l})-\operatorname{Var}_{\bar{l}}[\mathrm{E}(\boldsymbol{\Delta} \boldsymbol{l} \mid \overline{\boldsymbol{l}})]
$$

Due to the fact that each cycle constraint involves at least three elements of $\overline{\boldsymbol{l}}$ we do have pairwise independence between the $\bar{l}_{j}$, allowing us to calculate the variance of $\Delta l_{i}$ needed for $\sigma_{\Delta l}^{2}$ via Eq. 3.11, exactly:

$$
\sigma_{\Delta l_{i}}^{2}=\sum_{j=1}^{N z / 2} S_{i j}^{2} \sigma_{\bar{l}_{j}}^{2}=\sigma_{\bar{l}}^{2} \sum_{j=1}^{N z / 2} S_{i j}^{2}
$$

where the second equality follows from the fact that all $\bar{l}_{j}$ are equally distributed. Again, we use that $\mathbf{P}^{2}=\mathbf{P}$, hence $\mathbf{S}^{2}=-\mathbf{S}$, and therefore:

$$
\sum_{j=1}^{N z / 2} S_{i j}^{2}=-S_{i i} \Rightarrow \sigma_{\Delta l_{i}}^{2}=-S_{i i} \sigma_{\bar{l}}^{2} .
$$


From the above and Eqs. (3.6) and (3.13) it follows that

$$
\begin{aligned}
\sigma_{\Delta l}^{2} & =\frac{2}{N z} \sum_{i=1}^{N z / 2} \sigma_{\Delta l_{i}}^{2}=-\frac{2 \sigma_{\bar{l}}^{2}}{N z} \sum_{i=1}^{N z / 2} S_{i i} \\
& =-\frac{2 \sigma_{\bar{l}}^{2}}{N z} \operatorname{tr} \mathbf{S}=\frac{2}{z}\left(1-\frac{1}{N}\right) \sigma_{\bar{l}}^{2}
\end{aligned}
$$

Using Eq. 3.14 it further holds that $\operatorname{Var}_{\bar{l}}[\mathrm{E}(\boldsymbol{\Delta} \boldsymbol{l} \mid \overline{\boldsymbol{l}})]=(2 / z(1-1 / N))^{2} \operatorname{Var}(\bar{l})$ and therefore:

$$
\left\langle\sigma_{\Delta l \mid \bar{l}}^{2}\right\rangle_{\bar{l}}=\frac{2}{z}\left(1-\frac{1}{N}\right)\left[1-\frac{2}{z}\left(1-\frac{1}{N}\right)\right] \sigma_{\bar{l}}^{2}
$$

A natural question to ask is whether Eq. 3.19 ) can serve as an appropriate estimate for $\sigma_{\Delta l \mid \bar{l}}^{2}$. Empirically, we observe that $\sigma_{\Delta l \mid \bar{l}}^{2} \approx\left\langle\sigma_{\Delta l \mid \bar{l}}^{2}\right\rangle_{\bar{l}}$, given that $N \gg z$ (see Fig. 3.7 (c)). Furthermore, if additionally $z$ is sufficiently large, we indeed observe that $p_{\Delta l \mid \bar{l}}$ is approximately normally distributed (see Fig. 3.7(a)):

$$
p_{\Delta l \mid \bar{l}} \simeq \mathcal{N}\left(\mu_{\Delta l \mid \bar{l}},\left\langle\sigma_{\Delta l \mid \bar{l}}^{2}\right\rangle_{p_{\bar{l}}}\right)
$$

with the expressions for $\mu_{\Delta l \mid \bar{l}}$ and $\left\langle\sigma_{\Delta l \mid \bar{l}}^{2}\right\rangle_{p_{\bar{l}}}$ given in Eqs. 3.14 and 3.19.

Starting from an initial length distribution $p_{\bar{l}}$ with zero mean and variance $\sigma_{\bar{l}}^{2}=\sigma_{\bar{l}}^{2}$, we can use Eqs. (3.8) and (3.20) to derive the final length distribution $p_{l^{*}}$ in mechanical equilibrium. We apply this approach to our simulations by choosing $p_{\bar{l}}$ to be uniform; it follows that $\sigma_{\bar{l}}^{2}=1 / 12$. Eventually, we obtain:

$$
\begin{aligned}
p_{\boldsymbol{l}^{*}}(x) \simeq & \int_{-\infty}^{+\infty} \mathcal{U}(-0.5,0.5)(a) \cdot \mathcal{N}\left(\mu_{\Delta l \mid \bar{l}},\left\langle\sigma_{\Delta l \mid \bar{l}}^{2}\right\rangle\right)(x-a) d a \\
= & \frac{1}{\sqrt{2 \pi\left\langle\sigma_{\Delta l \mid \bar{l}}^{2}\right\rangle}} \int_{-0.5}^{0.5} \exp \left[-\left(\frac{x-\left(1-\frac{2}{z}\left(1-\frac{1}{N}\right)\right) a}{\left.\sqrt{2\left\langle\sigma_{\Delta l \mid \bar{l}}^{2}\right.}\right)^{2}}\right] d a\right. \\
= & {[2(1-2 / z(1-1 / N))]^{-1}\left[\operatorname{erf}\left(\frac{x+\left(1-\frac{2}{z}\left(1-\frac{1}{N}\right)\right) / 2}{\sqrt{2\left\langle\sigma_{\Delta l \mid \bar{l}}^{2}\right\rangle}}\right)\right.} \\
& \left.-\operatorname{erf}\left(\frac{x-\left(1-\frac{2}{z}\left(1-\frac{1}{N}\right)\right) / 2}{\sqrt{2\left\langle\sigma_{\Delta l \mid \bar{l}}^{2}\right\rangle}}\right)\right] .
\end{aligned}
$$

In Fig. 3.5 we compare this analytical expression to individual simulated networks. 
(a)
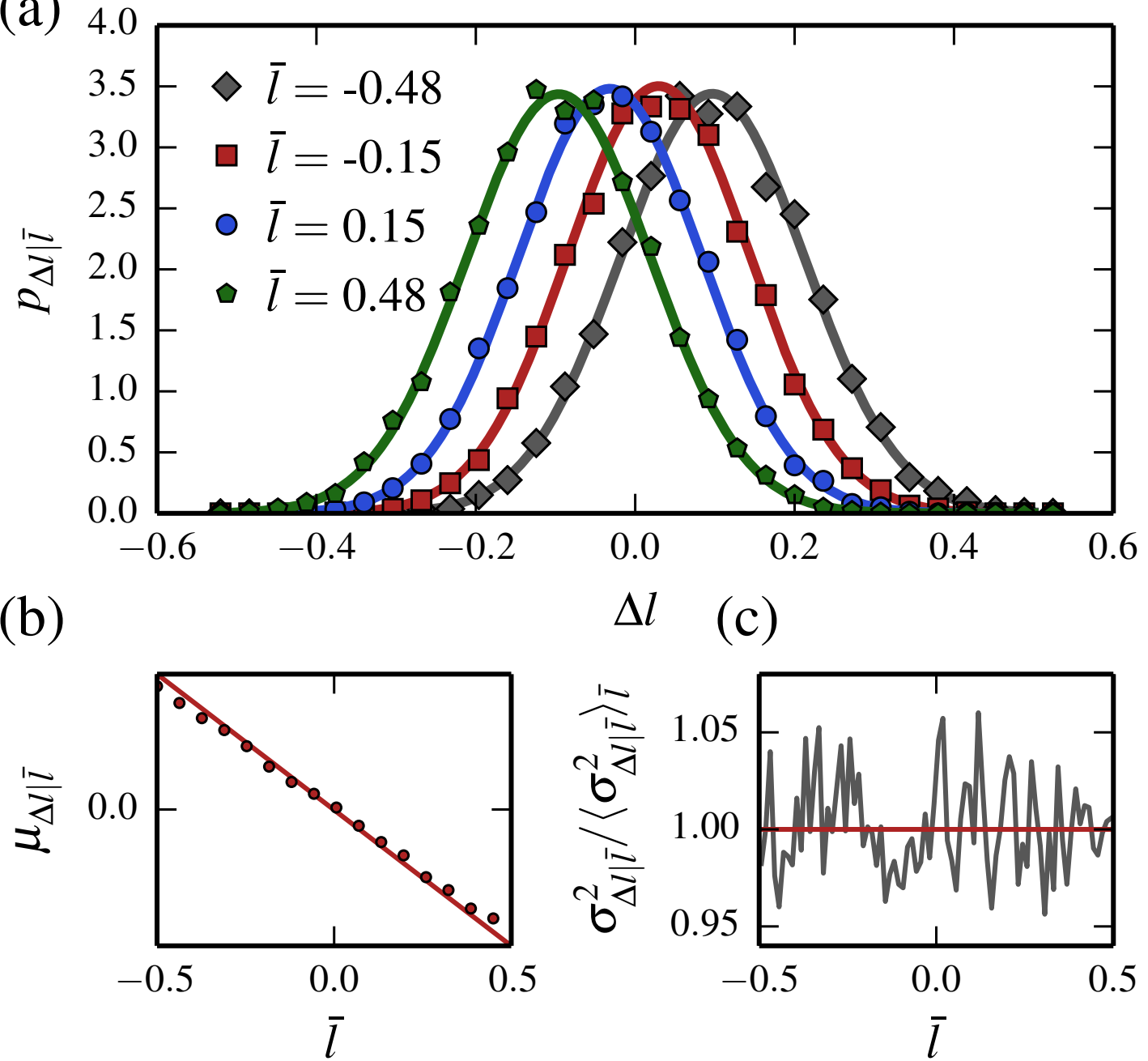

Figure 3.7.: Conditional quantities for an ensemble average over 50 simulation of random regular networks with $N=1000$ nodes and degree $z=10$. (a) Histograms $p_{\Delta l \mid \bar{l}}$ for various initial spring lengths $\bar{l}$; solid lines are Gaussian fits. (b) Conditional means $\mu_{\Delta l \mid \bar{l}}$ (solid circles) and theoretical prediction Eq. (3.14) (solid line). (c) Conditional variance $\sigma_{\Delta l \mid \bar{l}}$ over the theoretical value for the average variance $\mathrm{E}_{\bar{l}}\left[\sigma_{\Delta l \mid \bar{l}}^{2}\right]$ (see Eq. (3.19) as a function of $\bar{l}$. 
It is straightforward to generalize the approach to higher spatial dimensions $d$ if the probability distributions $p_{\bar{l}_{k}}$ for the components of the initial spring vectors are independent. In that case, due to the linear spring forces, the optimization problem decouples into the spatial components. The probability density for the final spring vectors then is simply given as the product of the one-dimensional results:

$$
p_{\boldsymbol{l}^{*}}(\mathbf{z})=\prod_{k=1}^{d} p_{\boldsymbol{l}_{k}^{*}}\left(z_{k}\right) .
$$

\subsubsection{Conclusions}

We present a probabilistic theory of length distributions in random spring networks on the unit circle. In a network with initially unbalanced forces at nodes, we can derive mean and variance of the expected distribution of length changes upon relaxation into mechanical equilibrium. Interestingly, the variance does not depend on the explicit network structure, but only on the number of nodes $N$ and average degree $z$. For a certain class of networks $(2<z \ll N)$, we derive a closed-form expression for the expected final length distribution that is in good agreement with numerical simulations.

Our modeling approach guarantees exact conservation of cycle contractibilities and therefore provides the appropriate framework for simulations of periodic spring-type networks. We are not aware of any other study that accounts for this necessary constraint for the preservation of network structure when using periodic boundary conditions.

This study represents a significant step in the direction of understanding the mechanical properties of polymer networks taking into account the entire network structure as opposed to, e.g., mean field approaches (see, e.g., 17 19]). However, in order to relate our results to elastic properties, one will need to introduce externally applied deformations, which have not been considered so far.

\section{References}

1. C. Heussinger \& E. Frey. Force distributions and force chains in random stiff fiber networks. Eur. Phys. J. E 24, 47-53 (2007). 
2. R. C. Arevalo, P. Kumar, J. S. Urbach \& D. L. Blair. Stress Heterogeneities in Sheared Type-I Collagen Networks Revealed by Boundary Stress Microscopy. PloS ONE 10, e0118021 (2015).

3. D. Mizuno, C. Tardin, C. F. Schmidt \& F. C. MacKintosh. Nonequilibrium mechanics of active cytoskeletal networks. Science 315, 370-373 (2007).

4. G. H. Koenderink et al. An active biopolymer network controlled by molecular motors. Proc. Natl. Acad. Sci. USA 106, 15192-15197 (2009).

5. J. Alvarado, M. Sheinman, A. Sharma, F. C. MacKintosh \& G. H. Koenderink. Molecular motors robustly drive active gels to a critically connected state. Nature Phys. 9, 591-597 (2013).

6. J. V. Shah \& P. A. Janmey. Strain hardening of fibrin gels and plasma clots. Rheol. Acta 36, 262-268 (1997).

7. W. A. Lam et al. Mechanics and contraction dynamics of single platelets and implications for clot stiffening. Nature Mater. 10, 61-66 (2011).

8. X. Trepat, G. Lenormand \& J. J. Fredberg. Universality in cell mechanics. Soft Matter 4, 1750-1759 (2008).

9. C. Heussinger. Stress relaxation through crosslink unbinding in cytoskeletal networks. New J. Phys. 14, 095029 (2012).

10. M. P. Murrell \& M. L. Gardel. F-actin buckling coordinates contractility and severing in a biomimetic actomyosin cortex. Proc. Natl. Acad. Sci. USA 109, 20820-20825 (2012).

11. P. Onck, T. Koeman, T. Van Dillen \& E. Van der Giessen. Alternative explanation of stiffening in cross-linked semiflexible networks. Phys. Rev. Lett. 95, 178102 (2005).

12. C. Heussinger \& E. Frey. Floppy modes and nonaffine deformations in random fiber networks. Phys. Rev. Lett. 97, 105501 (2006).

13. A. Sharma et al. Strain-controlled criticality governs the nonlinear mechanics of fibre networks. Nature Physics, advance online publication (2016).

14. A. S. G. van Oosten et al. Uncoupling shear and uniaxial elastic moduli of semiflexible biopolymer networks: compression-softening and stretch-stiffening. Sci. Rep. (2016). 
15. A. Wächter \& L. T. Biegler. On the implementation of an interior-point filter line-search algorithm for large-scale nonlinear programming. Math. Prog. 106, 25-57 (2005).

16. N. A. Weiss. A course in probability (Pearson/Addison-Wesley, 2006).

17. C. P. Broedersz, C. Storm \& F. C. MacKintosh. Nonlinear elasticity of composite networks of stiff biopolymers with flexible linkers. Phys. Rev. Lett. 101, 118103 (2008).

18. A. Sharma, M. Sheinman, K. M. Heidemann \& F. C. MacKintosh. Elastic response of filamentous networks with compliant crosslinks. Phys. Rev. E: Stat., Nonlinear, Soft Matter Phys. 88, 052705 (2013).

19. K. M. Heidemann, A. Sharma, F. Rehfeldt, C. F. Schmidt \& M. Wardetzky. Elasticity of 3D networks with rigid filaments and compliant crosslinks. Soft Matter 11, 343-354 (2015). 


\section{Summary \& Discussion}

In the course of this thesis, we developed a variety of modeling approaches to gain a comprehensive understanding of the different facets of the elastic properties of biopolymer networks. In general, modeling the mechanics of polymer networks is a formidable task. They are composed of various constituents, which themselves possess specific elastic properties. Describing the collective response of a network of interconnected, and therefore correlated, elements is far from trivial. The unifying scheme in our studies was the discussion of two different modeling paradigms: (i) continuum models that do not take into account the network structure explicitly but give access to analytical theory; and (ii) network models that consider the entire network and thereby take into account the inherent discrete nature of the system.

In Chapter 2, we studied networks of rigid rods connected by flexible crosslinks modeled as wormlike chains (WLC). We developed a continuum model to describe the elastic response under the assumption of affine deformations. In the limit of infinite crosslink density, we could analytically derive asymptotic power law scaling exponents for the differential elastic modulus as a function of shear stress. We found that the exponents depend on the dimensionality of the system. However, for any finite crosslink density, the only asymptotic power law scaling is that of the single WLC. We showed that this is due to the diverging force-extension relation of the WLC crosslinker. Our results are in stark contrast to studies by Broedersz et al. 44, 45], which predict a linear scaling of the modulus with stress. We argued that this behavior is inconsistent with the finite contour length of the crosslinks, since it would imply finite stress at any strain. Moreover, we set up efficient quasistatic simulations of three-dimensional networks of rigid rods with WLC crosslinkers. The results are in good agreement with our affine theory. However, we observed that the transition to the scaling behavior of a single WLC happened at smaller stresses than in the affine theory. This effect can be partly attributed to a network phenomenon called force chains. While approaching the maximum strain, system-spanning onedimensional paths develop that are stretched out and carry most of the system's 
energy. Eventually, there is only one path left that dominates the elastic response of the network and leads to the single WLC scaling of the modulus with stress. This effect is not captured by the affine theory since it neglects the specific network connectivity.

In summary, the simulations of networks with rigid rods and flexible WLC crosslinks lead to the insight that the accepted affine theory 44, 45 for this system had to be reconsidered. The development of a modified theoretical description 15, 16 showed that one has to distinguish between the continuum picture and a formulation with finite crosslink density that is in accordance with the simulations. Lastly, we realized that network topology plays an important role, when we observed the emergence of force chains. These can be of particular importance when rupture or unbinding events are taken into account. It would be interesting to study how stress redistributes after a force chain rupture event. Furthermore, it is unclear whether it is possible to determine, just based on the initial undeformed network configuration, which path in the network will eventually develop into the dominating force chain. Another natural extension to the model would be the inclusion of network dynamics, possibly in concatenation with crosslink (un)binding, which would give access to a, potentially, complex frequency response (see, e.g., [51]).

In Chapter 3 , we turned to a simpler model system. Instead of studying networks of rods and nonlinear springs, we described a network of linear springs on the unit circle. The network living on the circle is the one-dimensional variant of the common scheme of periodic boundary conditions. We derived a formulation for the optimization problem of finding the network configuration in force balance given a graph representation of the network, with the edge weights being the initial spring lengths after network generation. In particular, the network's cycles play an important role due to the fact that they can either be contractible or not. We are not aware of any other study that takes into account the necessity of preserving cycle contractibility during energy minimization. For a particular network realization, the problem admits an unique analytical solution. However, we aimed at more general, statistical statements. Using graph theory, we derived the variance of the expected distribution of spring length changes upon relaxation, while only fixing the number of nodes $N$ and the average degree $z$. Surprisingly, the variance does not depend on the particular graph/network under consideration, but only on $N$ and $z$. We further showed that application of a mean field approach, which neglects the graph structure and considers nodes as independent, leads to deviations from the correct solution, 
especially for sparsely connected networks. For networks with degree $2<z \ll N$ and initially uniform length distribution, we derived a closed-form expression for the full expected length distribution in mechanical equilibrium. The theoretical expression is in good agreement with numerical computations. For linear springs, the approach is easily generalizable to higher dimensions.

Our study of linear spring networks on the unit circle shows that it is possible to gain analytical results, and therefore conceptual insight, on mechanical properties of spring-type networks, even when following a full network approach. We further quantified to what extent a continuum description, i.e., neglecting network topology, deviates from these results.

The results on force distributions in spring networks could, potentially, be used for the study of network failure. The relation between topological network parameters, like degree of connectivity, and the response to internal forces, might allow for estimates on the probability of rupture events in a network due to internal stresses. Notice, that our discussion in Chapter 3 did not study the collective elastic response of the network under deformation. It remains to show how, if at all, our network approach can be extended in that direction.

Although linear spring networks on the circle might seem a very tractable problem, we realized that it admits a wealth of mathematical challenges, in terms of, e.g., statistical dependencies between initial spring lengths - spring lengths in cycles must always sum up to integer values.

If we now tried to capture the essence of this thesis in a few words, they would be the following: We attempted to disentangle which mechanical properties of an inherently discrete material, like a polymer network, can be described by continuum ideas and which mechanical properties need to be described, on the contrary, by the topology or finiteness of the number of elements in a material's composition. Hopefully, our application of diverse modeling and analysis approaches will be inspiring for future studies in this field. 



\section{A. Appendix}

This chapter contains derivations that are part of a master's thesis:

- K. M. Heidemann. Network elasticity of stiff rods connected by flexible linkers Master's Thesis (Georg-August-University Göttingen, 2012)

\section{A.1. The freely-jointed chain}

The freely-jointed chain (FJC) model describes a polymer as a noninteracting chain of $N$ segments of length $b$ with contour length $l_{0}=N b$. Its configuration therefore is defined by the set of bond vectors $\left\{\mathbf{r}_{1}, \mathbf{r}_{2}, \ldots, \mathbf{r}_{N}\right\}$, with $\left|\mathbf{r}_{i}\right|=b$ (see Fig. A.1). The orientations of distinct segments are statistically independent and random, meaning that

$$
\left\langle\mathbf{r}_{i}\right\rangle=0 \text { and }\left\langle\mathbf{r}_{i} \cdot \mathbf{r}_{j}\right\rangle=\left\{\begin{array}{ll}
b^{2}, & i=j \\
0, & i \neq j
\end{array},\right.
$$

$\langle\cdot\rangle$ denotes an ensemble average from statistical mechanics, meaning that we average over many copies of a single chain at a given instance of time, or equivalently, a time average of a single fluctuating chain! 1 . We define the end-to-end vector $\mathbf{R}:=\sum_{i=1}^{N} \mathbf{r}_{i}$ and calculate its probability distribution $P_{N}(\mathbf{R})$. We may now take advantage of the central limit theorem telling us that the probability distribtuion of a sum of $N$ independent random variables $Y=X_{1}+X_{2}+\cdots+X_{N}$, where all $X_{i}$ obey the same probability distribution with mean $\bar{X}$ and variance $\sigma_{X}^{2}$, approaches a Gaussian distribution for $N \gg 1$ :

$$
P(Y)=\frac{1}{\sqrt{2 \pi \sigma_{Y}^{2}}} \exp \left[-\frac{1}{\sqrt{2 \sigma_{Y}^{2}}}(Y-\bar{Y})^{2}\right],
$$

\footnotetext{
${ }^{1}$ if we assume ergodicity
} 


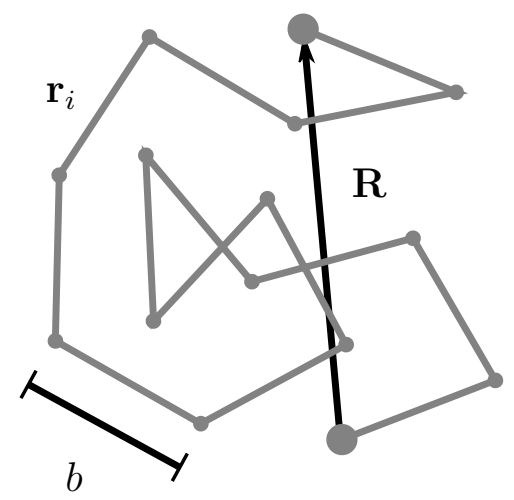

Figure A.1.: Arbitrary configuration of a freely-jointed chain with segment length $b$, bond vectors $\mathbf{r}_{i}$, and end-to-end vector $\mathbf{R}$.

with mean $\bar{Y}=N \bar{X}$ and variance $\sigma_{Y}^{2}=N \sigma_{X}^{2}$. In our case this means that $\langle\mathbf{R}\rangle=$ $N\left\langle\mathbf{r}_{i}\right\rangle=\mathbf{0}$ and $\left\langle\mathbf{R}^{2}\right\rangle=N\left\langle\mathbf{r}_{i}^{2}\right\rangle=N b^{2}$. Hence ${ }^{2}$

$$
P_{N}(\mathbf{R})=\left(\frac{3}{2 \pi N b^{2}}\right)^{3 / 2} \exp \left(-\frac{3|\mathbf{R}|^{2}}{2 N b^{2}}\right)
$$

The average end-to-end distance of a chain with $N$ bonds of length $b$ is given by $\sqrt{\left\langle\mathbf{R}^{2}\right\rangle}=\sqrt{N} b$. The scaling with the square root of the number of segments is typical for this kind of random walk statistics, e.g., the mean squared displacement of a diffusing particle scales with the time $t$. For derivations that are not based on the central limit theorem consult [10, 53].

Let us define $R:=|\mathbf{R}|$. When having a closer look at Eq. (A.3) it becomes obvious that the Gaussian approximation cannot be the whole truth. It predicts finite probability even for extensions $R>l_{0}$, so it is valid only for $R \ll l_{0}$.

As a next step we want to analyze which force is needed to stretch a FJC. In order to do so, we have to calculate the free energy of a FJC. It is defined via $F=U-T S$, where $T$ is the temperature of the surrounding reservoir, $U$ is the internal energy of the chain and $S$ its entropy. There is no internal energy assigned to the FJC but an entropy given by

$$
S=k_{\mathrm{B}} \ln \Omega(N, \mathbf{R}),
$$

\footnotetext{
${ }^{2}$ To be a little more accurate, one should use $\left\langle\mathbf{r}_{i}^{2}\right\rangle=b^{2}$ to show that for each cartesian component the variance is $b^{2} / 3$, as they are all independent. Then apply the central limit theorem to each of them and finally construct the multivariate Gaussian as a product of the univariates.
} 


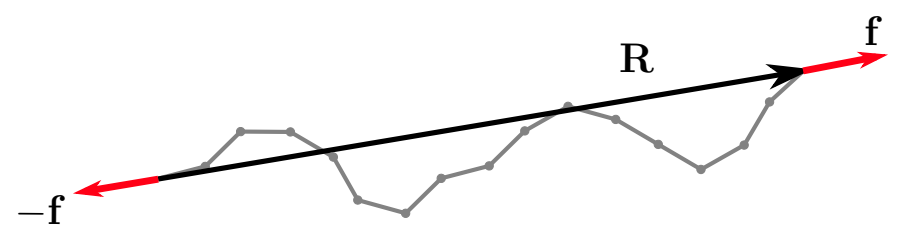

Figure A.2.: Freely-jointed chain with two opposing forces $(-\mathbf{f}, \mathbf{f})$ holding it at an end-to-end distance $R$.

with the number of accessible conformations $\Omega(N, \mathbf{R})$ for a chain with $N$ segments and end-to-end vector $\mathbf{R}$. It can be related to the probability distribution Eq. A.3. via

$$
P_{N}(\mathbf{R})=\frac{\Omega(N, \mathbf{R})}{\int \Omega(N, \mathbf{R}) d \mathbf{R}}
$$

The free energy of a FJC is therefore given as

$$
F=U-T S=\frac{3 R^{2}}{2 N b^{2}} k_{\mathrm{B}} T+\text { const } .
$$

The constant includes all terms that do not depend on $R$ since we are interested in changes of the free energy with respect to $R$ rather than in its actual value. Particularly, we calculate the force that is needed to hold the chain at a given end-to-end distance (see Fig. A.2), this is

$$
f=\frac{\partial F}{\partial R}=\frac{3 k_{\mathrm{B}} T}{N b^{2}} R
$$

Obviously the FJC in the Gaussian approximation behaves like a Hookean spring with spring constant $k=3 k_{\mathrm{B}} T / N b^{2}$. It becomes stiffer when the temperature increases. The chain wants to maximize its entropy but also has to work against the applied force. For larger temperatures the entropy becomes more dominant due to the $T$ factor in the free energy and so the effective spring constant increases. We should emphasize again that the Hookean spring result was derived from the Gaussian approximation and does only hold for small extensions. Nevertheless there is the possibility of extending the so-called force-extension relation to extensions approaching $l_{0}=N b$. This is done by calculating the free energy via the partition function (see [53]). For strong stretching one obtains

$$
\frac{f b}{k_{\mathrm{B}} T} \cong \frac{l_{0}}{l_{0}-\langle R\rangle}
$$




\section{A. Appendix}

So the force diverges as the extension approaches $l_{0}$, as expected.

\section{A.2. The wormlike chain}

Now we want to go from the coarse-grained, and therefore mathematically simpler, description of polymers to a more sophisticated model that takes into account cooperative coupling even on microscopic length scales, by introducing an elastic bending energy. One could think of additionally introducing stretching and twisting energies but for our purposes these will not be relevant. In the wormlike chain (WLC) model the polymer or rod is defined as a two times differentiable space curve $\mathbf{r}(s)$ parametrized by arc length $s$ with contour length $l_{0}$. The bending energy is defined via

$$
\mathcal{E}_{\text {bend }}(\{\mathbf{r}(s)\})=\frac{A}{2} \int_{0}^{l_{0}}\left|\frac{d \mathbf{t}}{d s}\right|^{2} d s=\frac{A}{2} \int_{0}^{l_{0}} \boldsymbol{\kappa}^{2} d s,
$$

where $A$ is the bending stiffness, $\mathbf{t}(s)=\mathbf{r}^{\prime}(s)$ is the unit tangent (arc length parametrization implies $\left|\mathbf{r}^{\prime}(s)\right|=|\mathbf{t}(s)|=1$ ) and $\boldsymbol{\kappa}=\mathbf{t}^{\prime}$ is the curvature vector. The persistence length is defined as $l_{\mathrm{p}}:=A /\left(k_{\mathrm{B}} T\right)$. It is the length over which the tangent vectors of the rod are decorrelating due to thermal motion, in particular it holds that $\left\langle\mathbf{t}(s) \cdot \mathbf{t}\left(s^{\prime}\right)\right\rangle=\exp \left(-\left|s-s^{\prime}\right| / l_{\mathrm{p}}\right)[19$.

Again we are interested in the response of the polymer to an externally applied force. In this case there is no straight forward way of calculating the free energy from the partition function due to the unit tangent constraint. Nevertheless there exists a mean-field approach deriving an expression for the free energy, which clearly distinguishes between an entropic and an energetic part [54]. We will follow an approach that has been applied by Marko \& Siggia [6] and can be found in [10] in more detail.

The aim is to find a force-extension relation for the WLC model that is exact in the limit of large forces and then to extend it by interpolation. As a first step we set up the Hamiltonian for a WLC that is subject to a constant external force acting on both ends of the chain. Assume that the force is pointing along the end-to-end vector $\mathbf{R}$, which will be our $x$-direction, such that we have $\mathbf{f}=f \hat{\mathbf{x}}$, where $f$ is the absolute value of the applied force and $\hat{\mathbf{x}}$ is a unit vector in $x$-direction (see Fig. A.3). The work that is done by the chain against this force is given by 


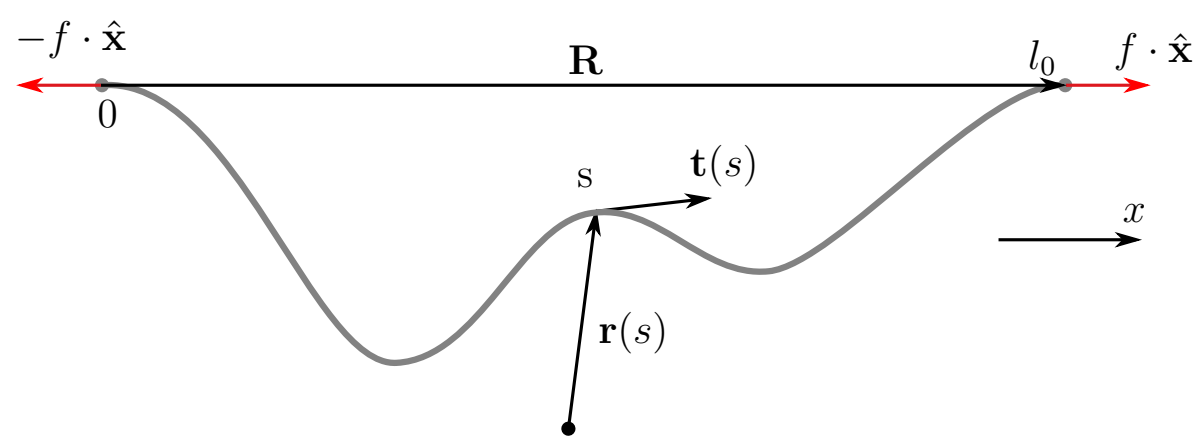

Figure A.3.: Schematic of a wormlike chain subject to a constant external force acting on both of its ends.

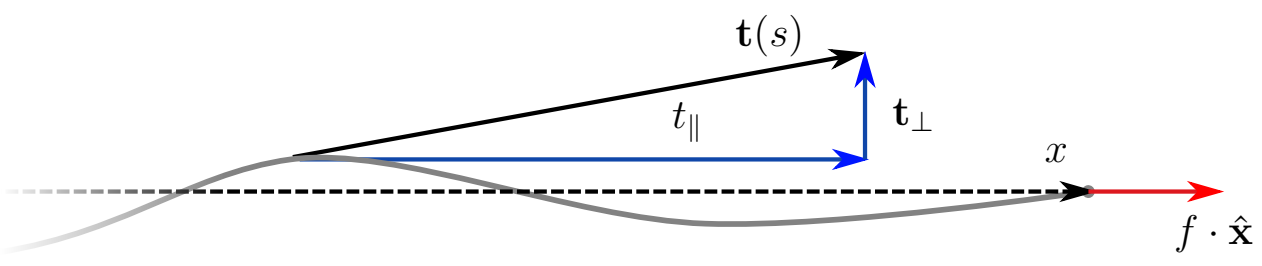

Figure A.4.: Decomposition of the unit tangent into components that are parallel/perpendicular to the $x$-direction.

$$
\mathcal{W}_{\text {ext }}(\{\mathbf{r}(s)\})=-f \hat{\mathbf{x}} \cdot \mathbf{R}=-f \hat{\mathbf{x}} \cdot \int_{0}^{l_{0}} \mathbf{t}(s) d s
$$

equipping us with the full Hamiltonian for a WLC under external force:

$$
\mathcal{H}(\{\mathbf{r}(s)\})=\frac{A}{2} \int_{0}^{l_{0}}\left|\frac{d \mathbf{t}(s)}{d s}\right|^{2} d s-f \hat{\mathbf{x}} \cdot \int_{0}^{l_{0}} \mathbf{t}(s) d s
$$

In order to apply our first approximation we decompose the unit tangent vector via $\mathbf{t}=\left(t_{\|}, \mathbf{t}_{\perp}\right)$, where $t_{\|}$and $\mathbf{t}_{\perp}$ are the components of $\mathbf{t}$ parallel and perpendicular to the $x$-axis (see Fig. A.4). Our first assumption is that $\mathbf{t}_{\perp}$ is much smaller than $t_{\|}$, meaning that the chain is only weakly bending and therefore $\mathbf{t}$ is almost parallel to 


\section{A. Appendix}

the $x$-axis. This is certainly true for sufficiently large forces $f$ or stiff polymers. Next we expand $\mathcal{H}$ in a Taylor series in $\mathbf{t}_{\perp}$ up to second order, in particular,

$$
\begin{gathered}
\hat{\mathbf{x}} \cdot \mathbf{t}=t_{\|}=\sqrt{1-\left|\mathbf{t}_{\perp}\right|^{2}} \approx 1-\frac{1}{2}\left|\mathbf{t}_{\perp}\right|^{2}, \\
\left|\frac{d \mathbf{t}}{d s}\right|^{2}=\left|\frac{d \mathbf{t}_{\|}}{d s}\right|^{2}+\left|\frac{d \mathbf{t}_{\perp}}{d s}\right|^{2} \approx\left|\frac{d \mathbf{t}_{\perp}}{d s}\right|^{2},
\end{gathered}
$$

which leads to the Hamiltonian:

$$
\mathcal{H}_{\perp}\left(\left\{\mathbf{t}_{\perp}(s)\right\}\right)=\int_{0}^{l_{0}}\left(\frac{A}{2}\left|\frac{d \mathbf{t}_{\perp}}{d s}\right|^{2}+\frac{f}{2}\left|\mathbf{t}_{\perp}\right|^{2}\right) d s-f l_{0} .
$$

For getting rid of the derivative we expand $\mathbf{t}_{\perp}(s)$ into a Fourier series on the interval $\left[0, l_{0}\right]$ in both spatial components, with the boundary conditions $t_{\perp}^{\prime}(0)=t_{\perp}^{\prime}\left(l_{0}\right)=0$ :

$$
\mathbf{t}_{\perp}(s)=\sum_{q} \mathbf{a}_{q} \cos (q s), \quad q=n \frac{\pi}{l_{0}}, n \in \mathbb{N}^{+},
$$

with Fourier coefficients $\mathbf{a}_{q}$ of $\mathbf{t}_{\perp}$ defined as

$$
\mathbf{a}_{q}=\frac{2}{l_{0}} \int_{0}^{l_{0}} \mathbf{t}_{\perp}(s) \cos (q s) d s .
$$

The coefficient $\mathbf{a}_{0}$ does not contribute because the end-to-end vector points along the $x$-axis, there is no 0th perpendicular component.

Substituting $\mathbf{t}_{\perp}(s)$ by its Fourier series in Eq. A.14 yields

$$
\mathcal{H}_{\perp}\left(\left\{\mathbf{a}_{q}\right\}\right)=\frac{l_{0}}{2} \sum_{q} \frac{1}{2}\left(A q^{2}+f\right)\left|\mathbf{a}_{q}\right|^{2}-f l_{0}
$$

where we have applied Parseval's theorem (see appendix A.3). Equation (A.17) now represents a harmonic Hamiltonian in the Fourier coefficients. If we assume that the chain is in equilibrium at temperature $T$ we can apply the equipartition theorem which states that in thermal equilibrium each degree of freedom, here the Fourier modes, that contributes quadratically to the system's Hamiltonian carries on average energy $k_{\mathrm{B}} T / 2$ (see appendix A.4 for derivation). It thus holds that

$$
\left\langle E_{q}\right\rangle=\frac{l_{0}}{4}\left(A q^{2}+f\right)\left\langle\left|\mathbf{a}_{q}\right|^{2}\right\rangle=d \cdot \frac{k_{\mathrm{B}} T}{2},
$$


where the factor $d$ is the dimensionality, in our case $d=2$ because $\mathbf{t}_{\perp}$ and therefore $\mathbf{a}_{q}$ have two components. If we solve for the Fourier coefficients we get

$$
\left\langle\left|\mathbf{a}_{q}\right|^{2}\right\rangle=\frac{4 k_{\mathrm{B}} T}{l_{0}\left(A q^{2}+f\right)}
$$

Remember, we are looking for a force-extension relation. Just as for the FJC we have constant force and have to calculate an average end-to-end distance. This is given by

$$
\begin{aligned}
& \langle R\rangle=\hat{\mathbf{x}} \cdot \int_{0}^{l_{0}}\langle\mathbf{t}\rangle d s=\int_{0}^{l_{0}}\left\langle\mathbf{t}_{\|}\right\rangle d s \approx \int_{0}^{l_{0}}\left(1-\frac{1}{2}\left\langle\left|\mathbf{t}_{\perp}\right|^{2}\right\rangle\right) d s
\end{aligned}
$$

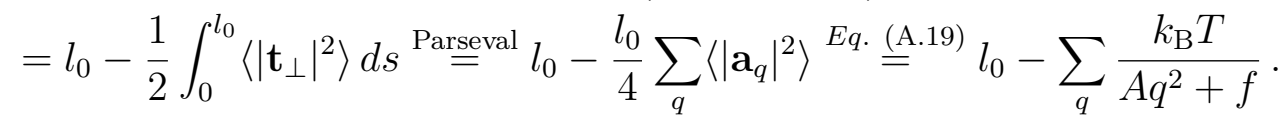

The sum in Eq. A.21) can be computed, the average deviation from maximum extension due to thermal fluctuations can be expressed as

$$
l_{0}-\langle R\rangle=\sum_{q} \frac{k_{\mathrm{B}} T}{A q^{2}+f}=k_{\mathrm{B}} T \frac{l_{0} \sqrt{f} \operatorname{coth}\left(\frac{l_{0} \sqrt{f}}{\sqrt{A}}\right)-\sqrt{A}}{2 f \sqrt{A}} .
$$

This is the force-extension relation for the WLC in the weakly bending approximation. Let us have a look at the limits of large and small forces. For small $x$ it holds that $\operatorname{coth}(x) \approx 1 / x+x / 3-x^{3} / 45$ and such

$$
\begin{aligned}
l_{0}-\langle R\rangle & \approx k_{\mathrm{B}} T \frac{l_{0}^{2}}{6 A}-k_{\mathrm{B}} T \frac{l_{0}^{4} f}{90 A^{2}} \\
\Rightarrow \quad f & =\frac{90 A^{2}}{k_{\mathrm{B}} T l_{0}^{4}} \delta l_{0} \quad \text { where } \quad \delta l_{0}:=\langle R\rangle-\left(l_{0}-k_{\mathrm{B}} T \frac{l_{0}^{2}}{6 A}\right) .
\end{aligned}
$$

The term in brackets in Eq. A.24) denotes the average end-to-end distance at zero force. Obviously there is again a linear relation between force and deviation from this end-to-end distance in the small force regime, just as we had seen for the FJC.

Now going to the high force regime we use $\lim _{x \rightarrow \infty} \operatorname{coth}(x)=1$ and get

$$
l_{0}-\langle R\rangle \approx \frac{k_{\mathrm{B}} T l_{0}}{2 \sqrt{A f}} \Rightarrow f \approx \frac{\left(k_{\mathrm{B}} T\right)^{2}}{A} \frac{l_{0}^{2}}{4\left(l_{0}-\langle R\rangle\right)^{2}}
$$




\section{A. Appendix}

The force diverges with $f \propto 1 /\left(l_{0}-\langle R\rangle\right)^{2}$ as the end-to-end distance approaches the contour length. This is in contrast to the FJC where we had $f \propto 1 /\left(l_{0}-\langle R\rangle\right)$. The reason for this discrepancy are the bending fluctuations on even smallest length scales that are allowed in the WLC model but not in the FJC, where the effective segment length $b$ (often called Kuhn length) is the smallest length scale that is involved. It is related to the persistence length via $b=2 l_{\mathrm{p}}[10]$.

Another interesting fact is the temperature dependence of the effective spring constant in the linear regime. For the purely entropic FJC it was directly proportional to $T$ (see Eq. A.7) ) whereas for the WLC it is inversely proportional to $T$, which might be counter intuitive. An important point is the interplay between the elastic energy and entropy; for larger $T$ the bending fluctuations without an applied force are larger and therefore one could argue that these are easier to "stretch out".

The final step consists of finding an adequate description for the force-extension relation of DNA, which we want to simulate in the end. An important parameter in this context is the ratio of contour length $l_{0}$ to persistence length $l_{\mathrm{p}}$. Polymers with $l_{0} / l_{\mathrm{p}} \gg 1$ are called flexible, $l_{0} / l_{\mathrm{p}} \ll 1$ means the polymer is stiff and polymers with $l_{0} / l_{\mathrm{p}} \sim 1$ are said to be semiflexible. Even though in our simulations and the accompanying experiments the DNA strands are more in the range $\sim 1 \mu \mathrm{m}$, still, for zero force the DNA should remain in a random coil state, meaning that the weakly bending approximation that we used for the WLC model cannot be applied. Instead we use the harmonic potential deduced for the FJC model in the Gaussian approximation, namely Eq. A.7):

$$
f=\frac{3 k_{\mathrm{B}} T}{2 l_{0} l_{\mathrm{p}}}\langle R\rangle,
$$

where we have applied $b=2 l_{\mathrm{p}}$ and $N b=l_{0}$. In the regime of large deformations we choose the WLC formulation, i.e., Eq. A.25 with $l_{\mathrm{p}}=A /\left(k_{\mathrm{B}} T\right)$, resulting in the interpolation formula formulated by Marko and Siggia [6]:

$$
\frac{f l_{\mathrm{p}}}{k_{\mathrm{B}} T}=\frac{\langle R\rangle}{l_{0}}+\frac{1}{4\left(1-\langle R\rangle / l_{0}\right)^{2}}-\frac{1}{4},
$$

which is asymptotically exact (to first order) for small and large extensions and which has been shown to give a good description of DNA force-extension behavior (see Fig. 1.4). 
Lastly we will compute a differential stiffness $k(x)$ for the WLC, with the relative extension $x:=\langle R\rangle / l_{0}$. Certainly, it will not be constant since the force-extension relation is nonlinear. Eq. A.25 tells us that

$$
f(x) \propto \frac{1}{(1-x)^{2}} \quad \Rightarrow \quad k(x):=\frac{d f}{d x} \propto \frac{1}{(1-x)^{3}} \propto f^{3 / 2} .
$$

This scaling relation will be useful when it comes to the elasticity of networks built from wormlike chains.

\section{A.3. Parseval's theorem}

We will check the special case of Parseval's theorem

$$
\begin{aligned}
\int_{0}^{L}\left(\frac{d \mathbf{t}_{\perp}(s)}{d s}\right)^{2} d s & =\frac{L}{2} \sum_{q}\left|\mathbf{a}_{q}\right|^{2} q^{2}, \\
\text { where } \quad \mathbf{t}_{\perp}(s) & =\sum_{q} \mathbf{a}_{q} \cos (q s), \quad q=n \frac{\pi}{L}, n \in \mathbb{N}^{+}
\end{aligned}
$$

which we applied in A.17). The following holds:

$$
\begin{aligned}
\int_{0}^{L}\left(\frac{d \mathbf{t}_{\perp}(s)}{d s}\right)^{2} d s & =\int_{0}^{L}\left(\sum_{q}-\mathbf{a}_{q} q \sin (q s)\right)^{2} d s \\
& =\int_{0}^{L}\left(\sum_{q_{1}} \sum_{q_{2}} \mathbf{a}_{q_{1}} q_{1} \sin \left(q_{1} s\right) \cdot \overline{\mathbf{a}_{q_{2}}} q_{2} \sin \left(q_{2} s\right)\right) d s \\
& =\sum_{q}\left|\mathbf{a}_{q}\right|^{2} q^{2} \int_{0}^{L} \sin ^{2}(q s) d s \\
& =\frac{L}{2} \sum_{q}\left|\mathbf{a}_{q}\right|^{2} q^{2} .
\end{aligned}
$$

\section{A.4. Equipartition theorem}

Let us consider a Hamiltonian with a quadratic degree of freedom, namely

$$
\mathcal{H}(u)=c u^{2}
$$




\section{A. Appendix}

In our case we had $u=\left|a_{q}\right|$ and $c=\frac{L}{4}\left(\kappa q^{2}+f\right)$. The average energy is given by

$$
\left\langle E_{q}\right\rangle=\frac{\int_{-\infty}^{+\infty} c u^{2} e^{-\beta c u^{2}} d u}{\int_{-\infty}^{+\infty} e^{-\beta c u^{2}} d u}=\beta^{-1} \frac{\int_{-\infty}^{+\infty} x^{2} e^{-x^{2}} d x}{\int_{-\infty}^{+\infty} e^{-x^{2}} d x}=k_{\mathrm{B}} T \frac{\sqrt{\pi} / 2}{\sqrt{\pi}}=\frac{k_{\mathrm{B}} T}{2} .
$$




\section{List of publications}

- A. Sharma, M. Sheinman, K. M. Heidemann \& F. C. MacKintosh. Elastic response of filamentous networks with compliant crosslinks. Phys. Rev. E: Stat., Nonlinear, Soft Matter Phys. 88, 052705 (2013)

- K. M. Heidemann, A. Sharma, F. Rehfeldt, C. F. Schmidt \& M. Wardetzky. Elasticity of 3D networks with rigid filaments and compliant crosslinks. Soft Matter 11, 343-354 (2015)

- K. M. Heidemann, A. O. Sageman-Furnas, A. Sharma, F. Rehfeldt, C. F. Schmidt \& M. Wardetzky. Random spring networks on the unit circle (in preparation) 



\section{Bibliography}

1. B. Alberts et al. Molecular Biology of the Cell: Fifth Edition (Garland Science, 2008).

2. R. Jones. Soft Condensed Matter (Oxford University Press, 2002).

3. E. Schrödinger. What is life?: With mind and matter and autobiographical sketches (Cambridge University Press, 1992).

4. B. Maher. Spring theory. Nature 448 (2007).

5. T. Wittmann. <http://www.cellimagelibrary.org/images/240> (2016).

6. J. F. Marko \& E. D. Siggia. Stretching DNA. Macromolecules 28, 8759-8770 (1995).

7. T. Odijk. Stiff Chains and Filaments under Tension. Macromolecules 28, 70167018 (1995).

8. P. Nelson, M. Radosavljević \& S. Bromberg. Biological Physics: Energy, Information, Life (WH Freeman and Company, 2008).

9. L. Landau \& E. Lifshitz. Elasticity theory (Pergamon Press, 1975).

10. T. Kawakatsu. Statistical physics of polymers: an introduction (Springer Verlag, 2004).

11. C. P. Brangwynne et al. Bending dynamics of fluctuating biopolymers probed by automated high-resolution filament tracking. Biophysical journal 93, 346-59 (2007).

12. C. P. Broedersz \& F. C. MacKintosh. Modeling semiflexible polymer networks. Reviews of Modern Physics 86, 995-1036 (2014).

13. O. Kratky \& G. Porod. Röntgenuntersuchung gelöster Fadenmoleküle. Recueil des Travaux Chimiques des Pays-Bas 68, 1106-1122 (1949).

14. C. Bustamante, J. F. Marko, E. D. Siggia \& S. Smith. Entropic elasticity of lambda-phage DNA. Science 265, 1599-1600 (1994). 
15. A. Sharma, M. Sheinman, K. M. Heidemann \& F. C. MacKintosh. Elastic response of filamentous networks with compliant crosslinks. Phys. Rev. E: Stat., Nonlinear, Soft Matter Phys. 88, 052705 (2013).

16. K. M. Heidemann, A. Sharma, F. Rehfeldt, C. F. Schmidt \& M. Wardetzky. Elasticity of 3D networks with rigid filaments and compliant crosslinks. Soft Matter 11, 343-354 (2015).

17. G. A. Holzapfel \& R. W. Ogden. Elasticity of biopolymer filaments. Acta Biomaterialia 9, 7320-7325 (2013).

18. K. Luby-Phelps. Cytoarchitecture and physical properties of cytoplasm: volume, viscosity, diffusion, intracellular surface area. International review of cytology 192, 189-221 (1999).

19. M. Doi \& S. Edwards. The theory of polymer dynamics (Oxford University Press, USA, 1988).

20. K. E. Kasza et al. The cell as a material. Curr. Opin. Cell Biol. 19, 101-7 (2007).

21. R. H. Ewoldt, A. E. Hosoi \& G. H. McKinley. New measures for characterizing nonlinear viscoelasticity in large amplitude oscillatory shear. Journal of Rheology 52, 1427 (2008).

22. C. Semmrich, R. J. Larsen \& A. R. Bausch. Nonlinear mechanics of entangled F-actin solutions. Soft Matter 4, 1675 (2008).

23. S. A. Rogers \& M. P. Lettinga. A sequence of physical processes determined and quantified in large-amplitude oscillatory shear (LAOS): Application to theoretical nonlinear models. Journal of Rheology 56, 1 (2011).

24. K. E. Kasza et al. Actin filament length tunes elasticity of flexibly cross-linked actin networks. Biophys. J. 99, 1091-100 (2010).

25. C. P. Broedersz et al. Measurement of nonlinear rheology of cross-linked biopolymer gels. Soft Matter 6, 4120 (2010).

26. K. E. Kasza et al. Nonlinear elasticity of stiff biopolymers connected by flexible linkers. Phys. Rev. E: Stat., Nonlinear, Soft Matter Phys. 79, 041928 (2009).

27. M. L. Gardel et al. Prestressed F-actin networks cross-linked by hinged filamins replicate mechanical properties of cells. Proc. Natl. Acad. Sci. U.S.A. 103, 1762-1767 (2006). 
28. Y.-C. Lin, G. H. Koenderink, F. C. MacKintosh \& D. A. Weitz. Control of non-linear elasticity in F-actin networks with microtubules. Soft Matter 7, 902 (2011).

29. M. L. Gardel et al. Scaling of F-Actin Network Rheology to Probe Single Filament Elasticity and Dynamics. Phys. Rev. Lett. 93, 1-4 (2004).

30. Y.-C. Lin, G. H. Koenderink, F. C. MacKintosh \& D. A. Weitz. Viscoelastic Properties of Microtubule Networks. Macromolecules 40, 7714-7720 (2007).

31. M. L. Gardel et al. Elastic behavior of cross-linked and bundled actin networks. Science 304, 1301-5 (2004).

32. M. J. Unterberger \& G. A. Holzapfel. Advances in the mechanical modeling of filamentous actin and its cross-linked networks on multiple scales. Biomechanics and modeling in mechanobiology (2014).

33. C. Storm, J. J. Pastore, F. C. Mackintosh, T. C. Lubensky \& P. A. Janmey. Nonlinear elasticity in biological gels. Nature 435, 191-194 (2005).

34. K. John, D. Caillerie, P. Peyla, A. Raoult \& C. Misbah. Nonlinear elasticity of cross-linked networks. Physical Review E 87, 042721 (2013).

35. S. Roy \& H. J. Qi. Micromechanical model for elasticity of the cell cytoskeleton. Physical Review E 77, 1-9 (2008).

36. J. Wilhelm \& E. Frey. Elasticity of Stiff Polymer Networks. Phys. Rev. Lett. 91, 108103 (2003).

37. D. A. Head, A. J. Levine \& F. C. MacKintosh. Deformation of Cross-Linked Semiflexible Polymer Networks. Phys. Rev. Lett. 91, 2-5 (2003).

38. D. A. Head, A. Levine \& F. C. MacKintosh. Distinct regimes of elastic response and deformation modes of cross-linked cytoskeletal and semiflexible polymer networks. Phys. Rev. E: Stat., Nonlinear, Soft Matter Phys. 68, 1-16 (2003).

39. P. R. Onck, T. Koeman, T. van Dillen \& E. van der Giessen. Alternative Explanation of Stiffening in Cross-Linked Semiflexible Networks. Phys. Rev. Lett. 95, 19-22 (2005).

40. C. P. Broedersz, X. Mao, T. C. Lubensky \& F. C. MacKintosh. Criticality and isostaticity in fibre networks. Nature Physics 7, 983-988 (2011).

41. E. Huisman, C. Storm \& G. Barkema. Monte Carlo study of multiply crosslinked semiflexible polymer networks. Physical Review E 78, 1-11 (2008). 
42. H. E. Amuasi, C. Heussinger, R. L. C. Vink \& A. Zippelius. Nonlinear and heterogeneous elasticity of multiply-crosslinked biopolymer networks. New Journal of Physics 17, 083035 (2015).

43. E. M. Huisman, C. Heussinger, C. Storm \& G. T. Barkema. Semiflexible Filamentous Composites. Phys. Rev. Lett. 105, 118101 (11 2010).

44. C. P. Broedersz, C. Storm \& F. C. MacKintosh. Nonlinear elasticity of composite networks of stiff biopolymers with flexible linkers. Phys. Rev. Lett. 101, 118103 (2008).

45. C. P. Broedersz, C. Storm \& F. C. MacKintosh. Effective-medium approach for stiff polymer networks with flexible cross-links. Phys. Rev. E: Stat., Nonlinear, Soft Matter Phys. 79, 61914 (2009).

46. C. Heussinger \& E. Frey. Force distributions and force chains in random stiff fiber networks. The European Physical Journal E, 1-8 (2007).

47. E. M. Huisman, T. van Dillen, P. R. Onck \& E. Van der Giessen. ThreeDimensional Cross-Linked F-Actin Networks: Relation between Network Architecture and Mechanical Behavior. Phys. Rev. Lett. 99, 2-5 (2007).

48. G. Žagar, P. R. Onck \& E. Van der Giessen. Elasticity of Rigidly Cross-Linked Networks of Athermal Filaments. Macromolecules 44, 7026-7033 (2011).

49. A. W. Lees \& S. F. Edwards. The computer study of transport processes under extreme conditions. J. Phys. C: Solid State Phys. 5, 1921-1928 (1972).

50. K. M. Heidemann et al. Random spring networks on the unit circle (in preparation).

51. K. W. Müller et al. Rheology of semiflexible bundle networks with transient linkers. Phys. Rev. Lett. 112, 1-5 (2014).

52. K. M. Heidemann. Network elasticity of stiff rods connected by flexible linkers Master's Thesis (Georg-August-University Göttingen, 2012).

53. M. Rubinstein \& R. H. Colby. Polymer physics (Oxford University Press, 2003).

54. J. R. Blundell \& E. M. Terentjev. Stretching Semiflexible Filaments and Their Networks. Macromolecules 42, 5388-5394 (2009). 


\section{Acknowledgments}

First, I would like to thank my supervisor, Prof. Max Wardetzky, for giving me the opportunity to pursue my $\mathrm{PhD}$ in his group. He gave me a lot of scientific freedom as well as a comfortable funding via the SFB 755, which is not to be taken for granted. I really appreciate his faith in the work of his group members. We had very fruitful discussions; he always got to the heart of the problem and contributed valuable conceptual ideas based on his background in mathematics. I appreciate his wonderful sense of humor, as well.

Second, I would like to thank my second supervisor, Prof. Christoph Schmidt. I am glad I could profit from his expertise and his astonishing physical intuition. He also got me into contact with our later collaborators in Amsterdam, which was essential for the success of this project. Furthermore, he encouraged me to attend numerous conferences, which I enjoyed a lot and which gave me the opportunity to present my work to the scientific community.

I would also like to thank Florian Rehfeldt, who was also involved in the same SFB project and gave valuable advice. Further thanks to Meenakshi Prabhune, who conducted the experiments in our project and who it was really enjoyable to work with.

I am grateful for the support by the group of Prof. Eitan Grinspun at Columbia University. They provided a very inspiring working environment and helped me with setting up my initial simulation code.

A major role is played by Abhinav Sharma, who I got to know as a postdoc in Fred MacKintosh's group at VU Amsterdam. Only together could we unravel the "hairy rod" mystery. I am very glad to have him as a collaborator and friend. Seldom, have I met somebody with so many ideas and such a bold sense of humor.

This brings me to our group, the "Discrete Differential Geometry Lab". I will not name all (former) members; I just want to say that it was really fun working with you guys. Although we mostly worked on different topics, I could always ask for help or inspiration. Special thanks goes to Henrik, for getting involved with the 
serious mathematics in my problems. And in particular, Andy, who, not only did an excellent job in reading the manuscript of this thesis, but also joined the "springs on the circle" project and contributed significantly to its success. I have to thank Max, again, for gathering so many nice people (not counting myself) around him. A doctoral thesis is always associated with a single person. However, I am glad that I can say that this one is the product of wonderful teamwork.

This brings me to my dear friends (including our group). Thank you so much for being part of my life. I think it is, at least for me, very important to balance intense thinking with other activities, like cooking, playing handball, cultural activities, going to the farmer's market, etc. All this I could only enjoy so much because I have you guys. Special thanks goes to Matthias who read the manuscript for this thesis and gave valuable advice. Moreover, I would like to thank Fabian for sharing some of his statistics expertise.

Last but by no means least I would like to thank my family for their generous support.

Again, I am very grateful that I had the opportunity to do my PhD under such wonderful circumstances. As far as what people say, it had a positive effect on my personal development. And even if it was just for staying in Göttingen for a little longer and making the acquaintance of certain people, it was very much worth it :-). 